\title{
METABOLIC REGULATION AND CRYOTOLERANCE OF IN VITRO-PRODUCED HOLSTEIN EMBRYOS
}

\author{
A Thesis \\ presented to \\ the Faculty of California Polytechnic State University, \\ San Luis Obispo
}

\author{
In Partial Fulfillment \\ of the Requirements for the Degree \\ Master of Science in Agriculture \\ with a Specialization in Animal Science
}

by

Melissa Ann Roberts

December 2016 
(C) 2016

Melissa Ann Roberts

ALL RIGHTS RESERVED 


\section{COMMITTEE MEMBERSHIP}

TITLE: $\quad$ Metabolic Regulation and Cryotolerance of In Vitro-

Produced Holstein Embryos

AUTHOR: $\quad$ Melissa Ann Roberts

DATE SUBMITTED: December 2016

COMMITTEE CHAIR: $\quad$ Fernando Campos-Chillón, M.S., D.V.M., Ph.D., D.A.C.T.

Associate Professor of Animal Sciences

COMMITTEE MEMBER: $\quad$ Moises Barceló-Fimbres, D.V.M., M.S., Ph.D.

Research Veterinarian, AniCell Biotech LLC

COMMITTEE MEMBER: Daniel Gunnar Peterson, Ph.D.

Professor of Animal Sciences 


\section{ABSTRACT \\ Metabolic Regulation and Cryotolerance of In Vitro-Produced Holstein Embryos \\ Melissa Ann Roberts}

In vitro production and transfer of embryos has become a common practice within the dairy industry to efficiently breed superior animals and meet the consumption demand of the growing population. Cyropreservation is necessary for the application of commercialized embryo transfer, however, in vitro-produced embryos show morphological and physiological defects which negatively impact their ability to withstand cryopreservation in comparison to their in vivo counterparts. These artifacts result from culture conditions that cause stress to the embryo during development, leading to an accumulation of intracellular lipids, mitochondrial dysfunction, and ultimately poor ability to withstand freezing and thawing. The objective of these studies was to examine the effects of various metabolic regulators on the viability and cryotolerance of in vitroproduced embryos. Pilot studies revealed that evaluating early (stage 6) versus late (stage 7) blastocysts did not affect the trend seen in results, nor did culturing embryos in continuous versus sequential media. From the main experiment performed, it was concluded that a combination of metabolic regulators decreased lipid content, improved cryopreservation survival, and lowered the percentage of apoptotic cells present after thawing. Conditioned media increased the blastocyst percentage, but did not produce superior quality embryos as measured by cryotolerance. Research concerning the metabolic needs of the preimplantation embryo must continue to determine more relevant markers of embryo quality in vitro.

Keywords: bovine, embryo, metabolism, cryopreservation 


\section{ACKNOWLEDGMENTS}

I am tremendously grateful for the guidance and support of Dr. Fernando CamposChillón. His work ethic and passion for learning are an inspiration, and undoubtedly the reason for my pursuit of continued education in reproductive science.

To Dr. Joy Altermatt I owe the development of critical and philosophical thought in the quest for truth, passion, and morality. Her unwavering confidence in my abilities guided me forward even when the path was unclear, and will continue to do so in the future.

I am forever indebted to Dr. Dan Peterson for the support and wisdom he so generously shared over the years. He held me to the highest of standards and encouraged me to do my best work no matter the circumstances, an invaluable asset for success.

Dr. Moises Barceló-Fimbres has taught me the significance of collaboration. His extensive knowledge of embryology, molecular biology, and experimental design and analysis was the framework for this thesis. He played an instrumental role in the execution of and learning from these projects.

We are shaped by our surroundings; I am thankful for the influence of my family, friends, professors, and classmates along this journey. 


\section{TABLE OF CONTENTS}

Page

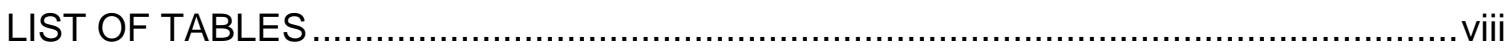

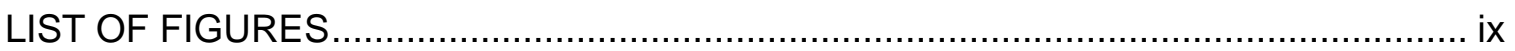

CHAPTER

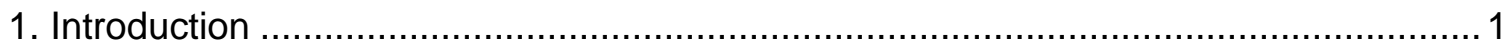

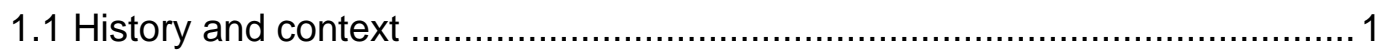

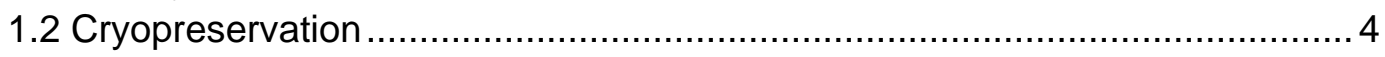

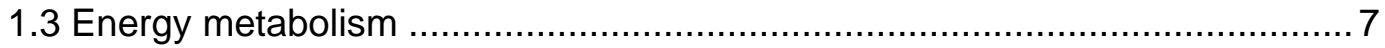

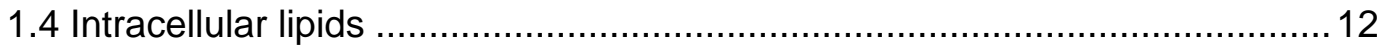

1.5 Mitochondrial function .......................................................................... 16

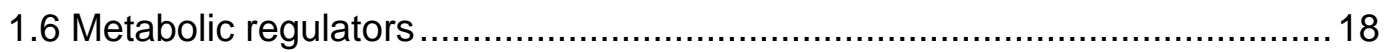

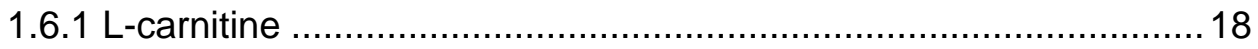

1.6.2 Forskolin.......................................................................... 18

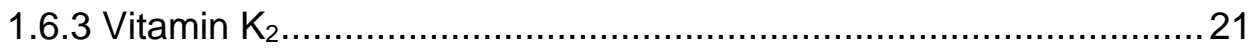

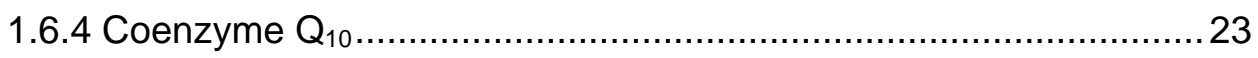

1.6.5 Conditioned media .............................................................. 23

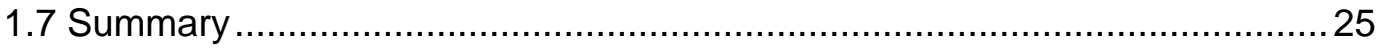

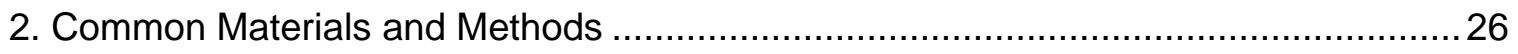

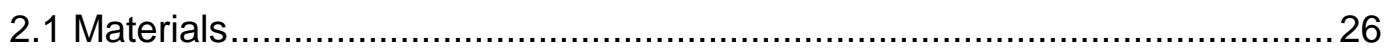

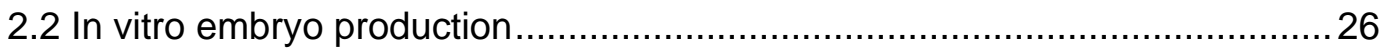

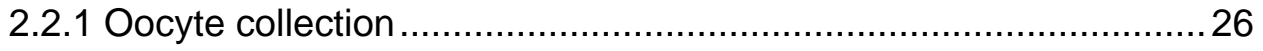

2.2 .2 In vitro maturation ................................................................ 26

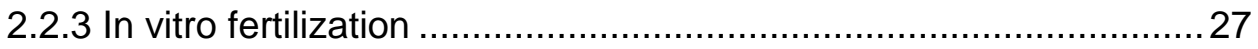

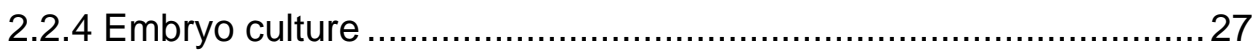

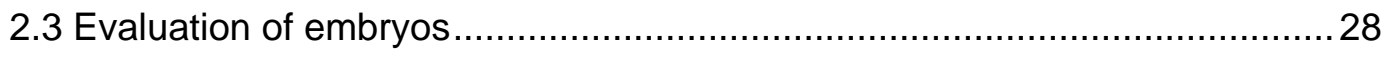

2.3.1 Cleavage and development .................................................... 28

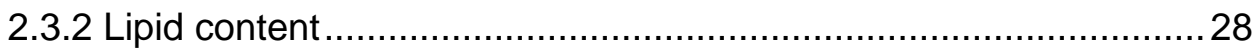

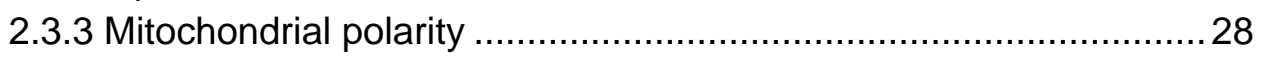

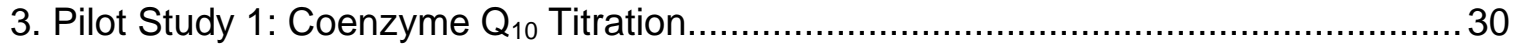

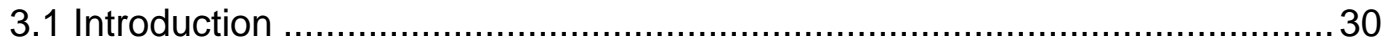

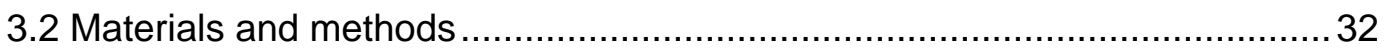

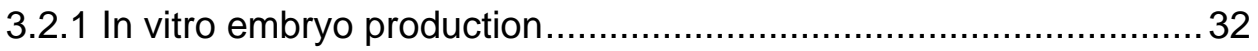

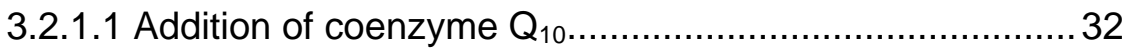

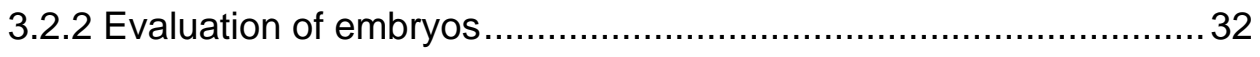

3.2.2.1 Embryo development ................................................ 32

3.2.2.2 Lipid content .......................................................... 32

3.2.2.3 Mitochondrial polarity ............................................ 33

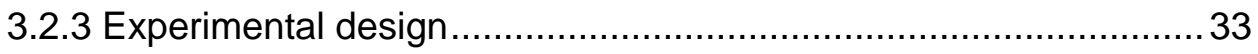

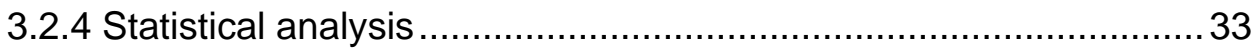

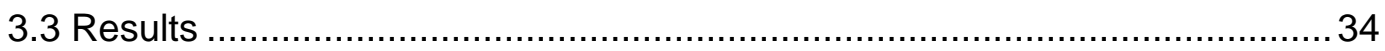

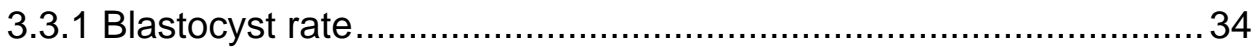




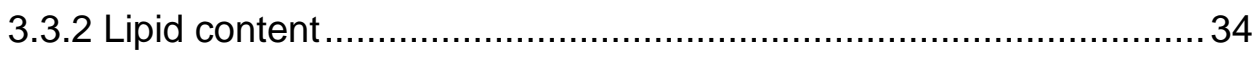

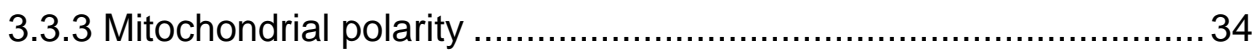

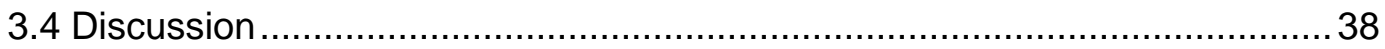

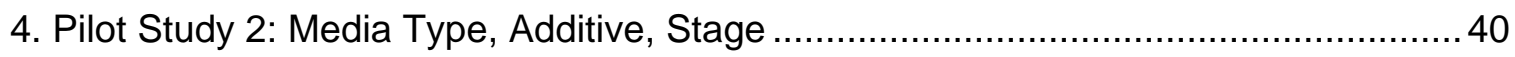

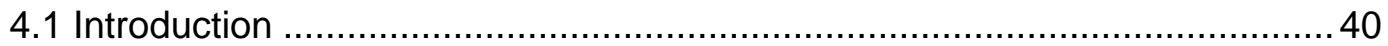

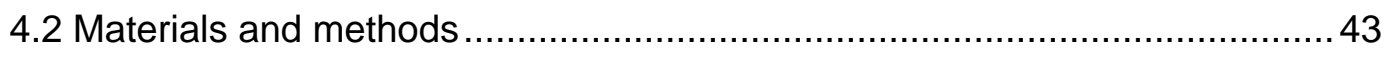

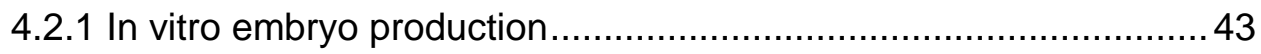

4.2.1.1 Addition of L-carnitine ................................................... 43

4.2.1.2 Addition of coenzyme Q10 ……………….................. 43

4.2.1.3 Addition of vitamin K2 ................................................ 43

4.2.1.4 Addition of forskolin ................................................. 43

4.2.2 Evaluation of embryos ............................................................ 43

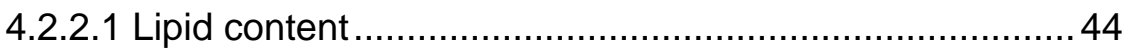

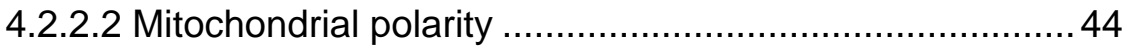

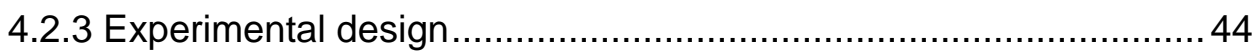

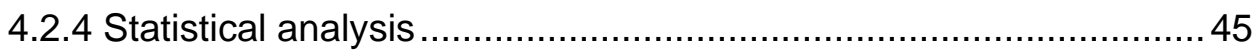

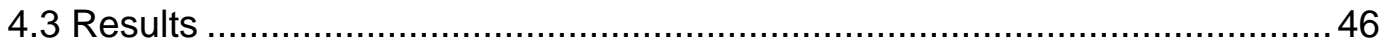

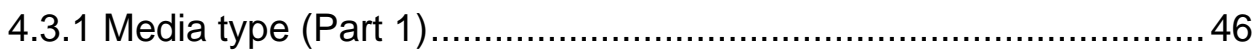

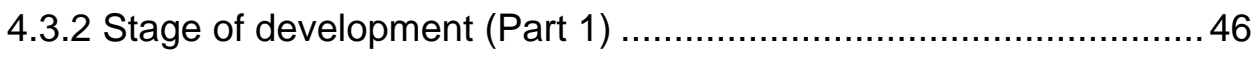

4.3.3 Media additives (Parts 1 and 2) ..................................................46

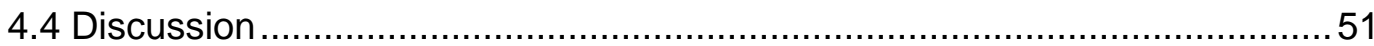

5. Experiment 1: Metabolic Regulators and Cryopreservation ....................................55

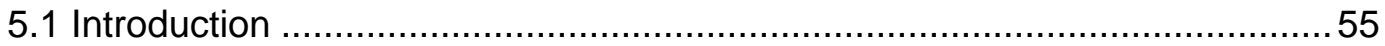

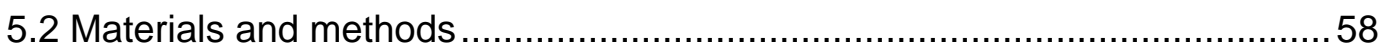

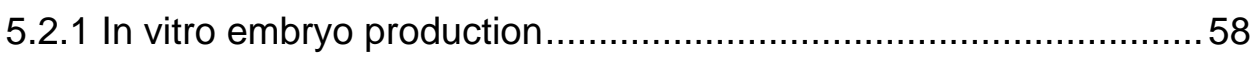

5.2.1.1 Addition of L-carnitine .............................................. 58

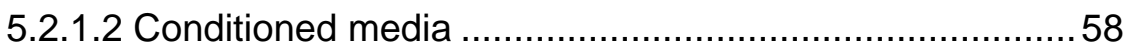

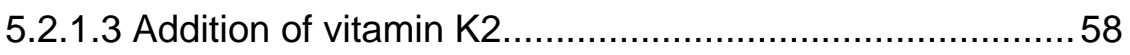

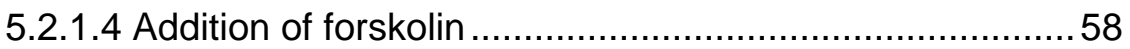

5.2.2 Cryopreservation and thawing .................................................59

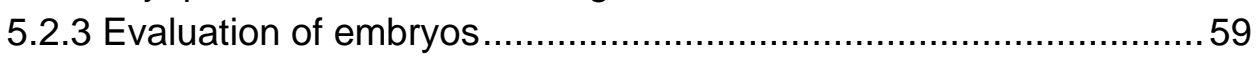

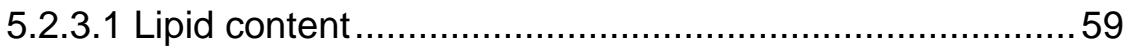

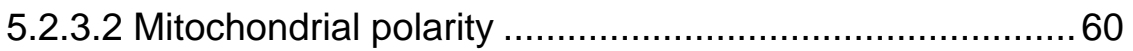

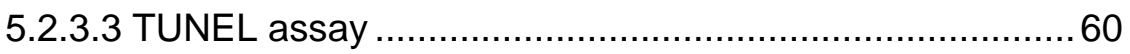

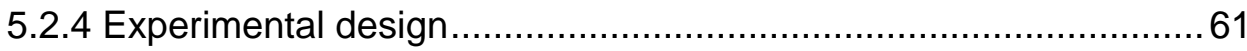

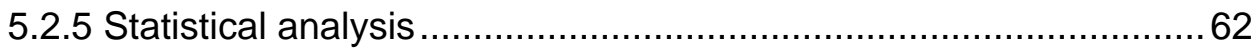

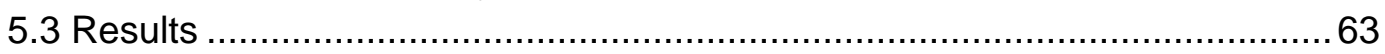

5.3.1 L-carnitine and conditioned medium (Part 1) ...............................63

5.3.2 Vitamin K2, forskolin, and conditioned medium (Part 2) …............63

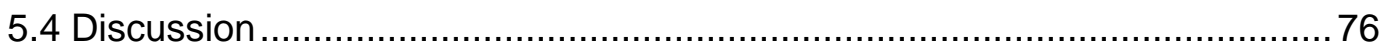

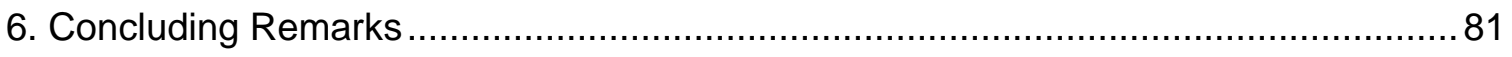

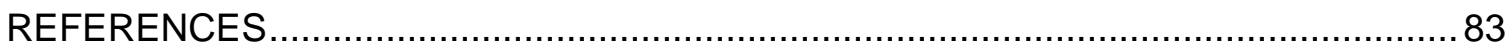




\section{LIST OF TABLES}

Table

Page

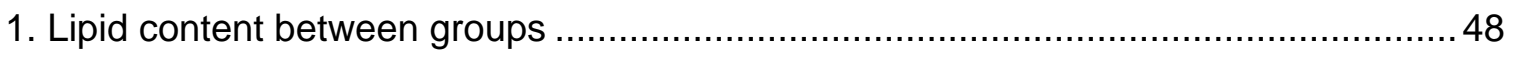




\section{LIST OF FIGURES}

Figure

1. Glucose metabolism within the cell .................................................................... 11

2. Fatty acid transport into the mitochondria and metabolic pathway ............................ 15

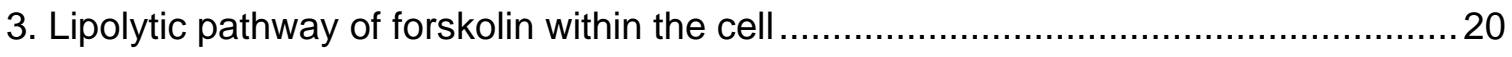

4. Proposed mechanism of vitamin $\mathrm{K}_{2}$ within the electron transport chain...................22

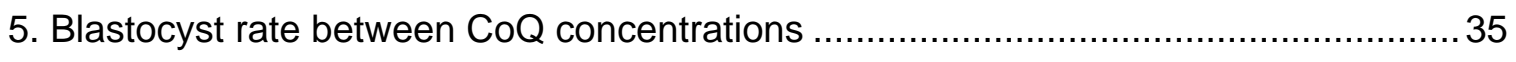

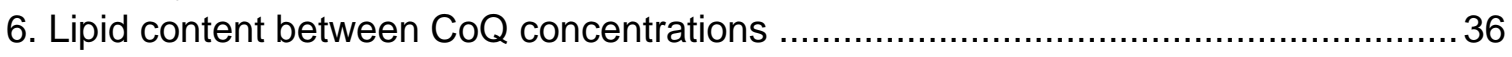

7. Mitochondrial polarity between CoQ concentrations ............................................ 37

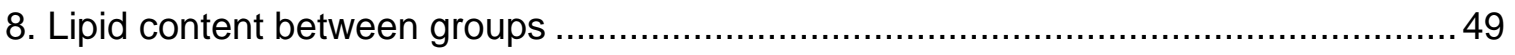

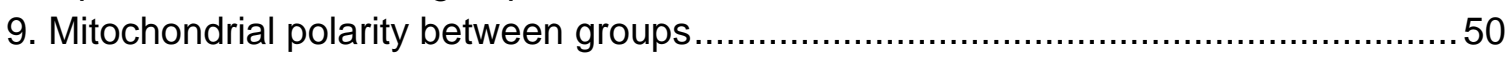

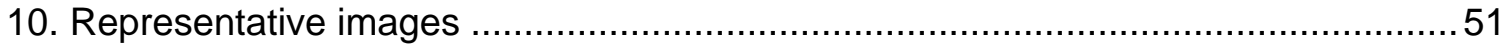

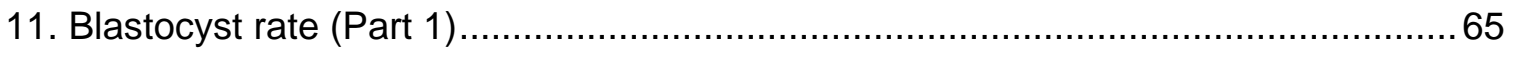

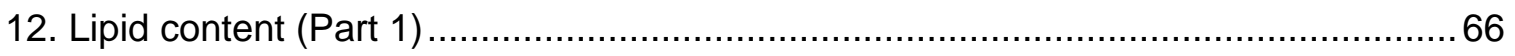

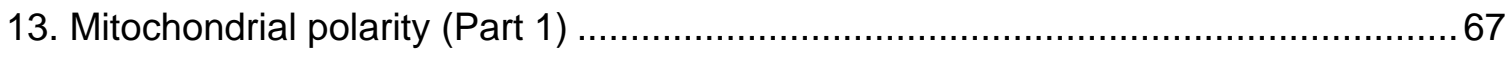

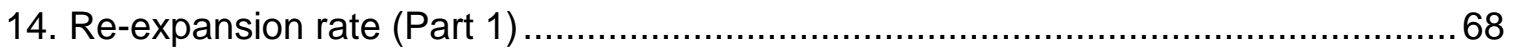

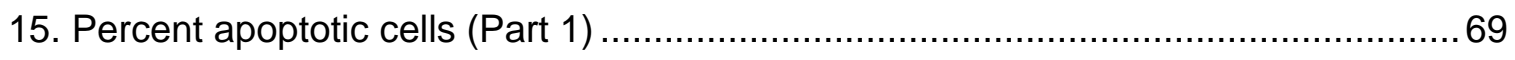

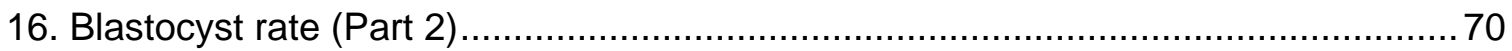

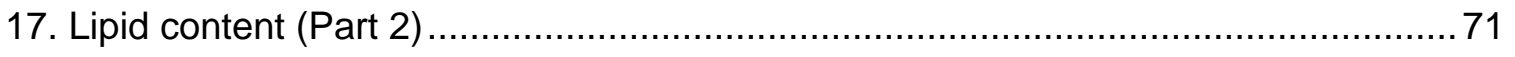

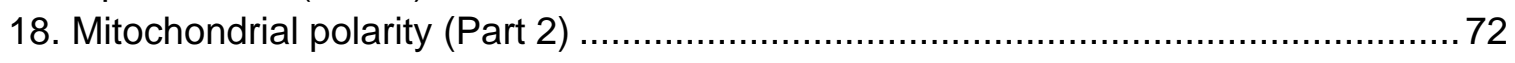

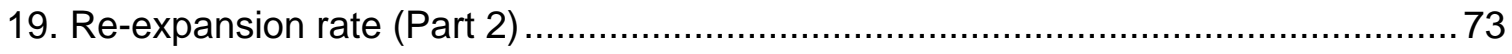

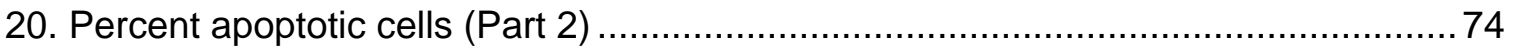

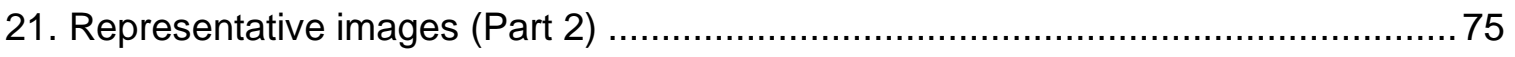




\section{Chapter 1. Introduction}

\subsection{History and context}

With a growing human population and limited supply of natural resources, the efficiency of milk production in the dairy industry becomes important. Genetic selection and breeding techniques using assisted reproductive technologies (ART) allow for the top producing cows to be efficiently bred, yielding an average of 6 to 7 viable embryos per cycle after superovulation, oocyte retrieval, and in vitro-fertilization (IVF) (Perry, 2014). These embryos can be transferred to recipients with an expected pregnancy rate of 50$60 \%$, and the process can be repeated with each cycle (Hasler, 2014). Using ART, multiple pregnancies per year can be generated from one superior genetic combination, compared to the typical 1 to 2 calves that a cow can naturally deliver per year. Therefore, fewer cows are needed to produce the same amount of milk, cutting down on feed costs, water requirements, and minimizing the carbon footprint of the dairy industry. These outcomes tackle some of the most pressing issues in the agricultural industry of the United States.

The downside of selective breeding is that primary focus has been placed on breeding for milk production rather than fertility, creating a subfertile population of dairy cattle (Moore \& Thatcher, 2006). This subfertility is characterized by low pregnancy rates and high embryonic mortality, with a final conception rate of only $28 \%$ per cycle in lactating dairy cows (Moore \& Thatcher, 2006). Multiple explanations and solutions for this occurrence have been proposed, but what has been made clear is that the dairy industry requires ART not only for genetic selection, but also for successful pregnancy outcomes. As research reveals the dynamics behind the reproductive cycle and early embryonic development in the cow, manipulation of these processes becomes practical.

Over the past 40 years, the practice of embryo transfer in dairy cattle has transformed from an experimental procedure into a reliable technique that serves as the basis for thousands of commercial businesses worldwide (Hasler, 2014). The success of 
embryo transfer brought about the advent of in vitro-produced (IVP) embryos, generated from oocytes that undergo IVF. These IVP embryos spend their beginning phase of life in an artificial setting aimed at mimicking the natural environment of a cow. The goal is to culture embryos until they develop to a stage in which they can be transferred nonsurgically into the uterus of a recipient, which is typically 7 days after fertilization.

Techniques such as superovulation and embryo transfer, in vitro production of embryos, fertilization using sexed semen, cloning, transgenesis, preimplantation genetic diagnostics, and gamete storage have arisen as the forefront assisted reproductive technologies (Moore \& Thatcher, 2006). The first live calf was produced by IVF in 1981, and the first live calf produced from in vitro-maturation (IVM) of an oocyte followed by IVF was born in 1986 (Moore \& Thatcher, 2006). IVF and embryo transfer has benefited agricultural business by allowing for the import and export of embryos between countries without the transmission of infectious disease (Mapletoft, 2013). Embryo production by IVF reached an all-time high in 2013, resulting in the transfer of over 400,000 embryos (Perry, 2014), and accounting for $42 \%$ of the total number of bovine embryos (in vitro plus in vivo) produced that year (Blondin, 2015). The future of embryo transfer holds the potential of genomic testing that will provide a selective means of transfer and lead to a more technologically sophisticated industry (Blondin, 2015; Hasler, 2014).

Despite the promising improvement of embryo culture and transfer on pregnancy rates of herds with low fertility, there are limitations that have stifled its progress. It is widely agreed that in vitro culture (IVC) of embryos does not accurately mimic the natural environment of the oviduct and uterus of a cow during pregnancy (Absalón-Medina, Butler, \& Gilbert, 2014; J. G. Thompson, 2000). Additionally, aside from the increased cost of ART as compared to standard artificial insemination (AI), the sub-optimal culture conditions of IVC have led to decreased fetal survival and occasional abnormalities (J. G. Thompson, 2000). One of the most noted abnormalities is known as "large offspring 
syndrome," and was linked to the use of serum in embryo culture media (Young, Sinclair, \& Wilmut, 1998). Furthermore, IVP bovine embryos have morphological, cellular, and physiological differences that impair their ability to withstand cryopreservation (Fair et al., 2001). These differences highlight the need for improvement of the IVC process to make IVP embryos more similar to their in vivo counterparts. 


\subsection{Cryopreservation}

Successful cryopreservation is essential for the use of IVP embryos in a commercial setting because it allows for them to be stored, shipped, and transferred to a recipient at the most opportune time (Fair et al., 2001; M. Sudano, Paschoal, \& Maziero, 2013). Beyond the commercial application of cryopreservation, this technology also provides a way to prevent permanent germplasm losses in the cattle population (Dobrinsky, 2002). In the early 2000s, foot-and-mouth disease outbreaks occurred in the U.S. and the U.K. then quickly spread to other parts of Europe, leading to the slaughter of over 4 million cows in the U.K. in an attempt to control the outbreak (Dobrinsky, 2002). By retaining a store of cryopreserved semen, oocytes, and embryos, the loss of these germplasm resources can be avoided in the event of disease outbreak or other catastrophes.

The most common method of cryopreservation is a slow freezing procedure which permits direct transfer of embryos into a recipient as the thawing process can occur in utero. This technique was first performed with a mouse embryo in 1972 (Whittingham, Leibo, \& Mazur, 1972), then shortly after with a bovine embryo in 1973 (Wilmut \& Rowson, 1973). In this procedure, the embryo is exposed to a low concentration (1.5M) of a permeating cryoprotectant, such as ethylene glycol or glycerol, then cooled to between 30 and $-65^{\circ} \mathrm{C}$ at a rate of $0.3-0.5^{\circ} \mathrm{C} / \mathrm{min}$, and finally plunged into liquid nitrogen (Saragusty \& Arav, 2011). During this slow decrease in temperature, permeating cryoprotectants enter the cell as water exits, localizing the formation of ice crystals to the extracellular solution as the intracellular matrix reaches a temperature at which it forms a solid (Mazur, 1963). Dehydration is not complete, however, and ice crystals can form within the cell, damaging organelles and causing cell death (Fahy, MacFarlane, Angell, \& Meryman, 1984; Gupta, Singh, \& Anzar, 2016; Mazur, 1963). Cryopreservation is significantly less 
successful in bovine embryos produced in vitro as compared to those produced in vivo (Enright et al., 2000).

Vitrification was introduced as an alternative to slow freezing because it transforms the intracellular matrix into a non-crystalline amorphous solid, eliminating the event of intracellular crystallization (Fahy et al., 1984). This glass-like state is induced as a result of an extreme elevation in viscosity in the presence of a high concentration (40\% w:v) of cryoprotectant (Fahy et al., 1984; Massip, 2001). After the embryo is dehydrated by culture with non-permeating cryoprotectants such as sucrose, the cytoplasm supercools until it vitrifies, thereby halting cellular processes while maintaining the ionic and molecular distribution of the liquid state (Massip, 2001; Mazur, 1963; Vajta \& Kuwayama, 2006). As a result, mechanical and chemical damage to the cell is avoided (Massip, 2001). While vitrification yields a higher post-warm survival of embryos (Dobrinsky, 2002; Gupta et al., 2016; Massip, 2001; Vajta \& Kuwayama, 2006), there is evidence that exposure to high concentrations of cryoprotective agents causes irreplaceable damage to the cytoskeleton and mitochondria of the embryo and can cause osmotic shock in the cells (Dobrinsky, 2002; Massip, 2001; Vajta \& Kuwayama, 2006).

Both slow freezing and vitrification techniques expose embryos to cryoprotectants and extreme temperature change, which are detrimental to embryo survival. There are two approaches to improving survival following cryopreservation: 1) modify the cryopreservation technique; or 2) modify the IVC technique to create a more resilient embryo (Dobrinsky, 2002; Seidel, 2006). Initial efforts focused on evolving the slow freezing technique. Determining the optimum cooling rate, cryoprotectant, thawing procedure, stage of embryo to freeze, and developing a direct transfer method allowed for moderate improvement in commercial application of this procedure (Massip, 2001). However, pregnancy rates of frozen embryos remains lower than optimum (Massip, 2001). Because the very qualities that decrease cryotolerance seem to be a result of 
inefficiencies in culture conditions, research has been directed toward improving the IVC system.

There are a number of structural and functional differences between in vivoderived and IVP embryos that contribute to the multi-functional event of cryosurvival (M. Sudano et al., 2013). In vivo-derived blastocysts are characterized by having a narrow perivitelline space, plasma membranes with a continuous cover of numerous stacked microvilli, a well-defined system of cell-to-cell coupling and a large population of round or elongated mitochondria with extensive transverse cristae (Fair et al., 2001); conversely, IVP blastocysts are characterized by a wider perivitelline space, no stacking of microvilli, decreased junctional contacts between trophoblast cells, and a notable increase in cytoplasmic lipid droplets (Fair et al., 2001). An accrual of intracellular lipids not only makes embryos more susceptible to oxidative damage (McEvoy, Robinson, \& Sinclair, 2001), but also impairs their ability to survive the phase changes that occur in the freezethaw cycle (Seidel, 2006).

It is not only the origin of embryos (in vivo vs. IVP) that determines their pregnancy rate after transfer, but also the act of slow freezing itself. One piece of evidence that points to invisible qualitative differences between fresh and frozen-thawed embryos is the difference in pregnancy rates following transfer of embryos of equal grade (Hasler, 2001). The transfer of grade 1 embryos of in vivo origin that have not been frozen $(n=4163)$ resulted in a $73.2 \%$ pregnancy rate, as compared to a $62.8 \%$ pregnancy rate of grade 1 embryos of in vivo origin that had been frozen and thawed ( $n=2482)$ (Hasler, 2001). Cryotolerance may be used as a tool for the ultimate measure of embryo quality (Dobrinsky, 2002; Seidel, 2006). 


\subsection{Energy metabolism}

As a fertilized oocyte develops, it proceeds through various stages of energy requirement. There are two mechanisms for ATP production in the pre-implantation embryo: oxidative phosphorylation and glycolysis (Jeremy G Thompson, Partridge, Houghton, Cox, \& Leese, 1996). During the initiation and continuation of cleavage, bovine embryos rely on pyruvate as an energy substrate for oxidative phosphorylation as the glycolytic pathway is limited (H. J. Leese, 2012; Rieger, 1992). Other substrates that have been studied as sources of energy throughout development are amino acids, lactate, and endogenous fatty acid stores derived from triglyceride (H. J. Leese, 2012; R. G. Sturmey, Brison, \& Leese, 2008). During genome activation, the glycolytic pathway begins to increase in function as glucose becomes the primary energy substrate, an occurrence which may be explained by the synthesis of one or more key glycolytic enzymes, such as hexokinase and phosphofructokinase, beginning at genome activation [Fig. 1] (Gardner, Pool, \& Lane, 2000; Rieger, 1992). Genome activation occurs between the 8 and 12-cell stage in the bovine embryo (Telford, Watson, \& Schultz, 1990).

Energy requirements and oxygen uptake remain fairly constant from the 1-cell to 8-cell stage; however, ATP production, oxygen consumption, glucose uptake, and oxygen uptake all increase at the morula stage (Jeremy G Thompson et al., 1996). The utilization of anaerobic respiration for ATP production is important between genome activation and blastocoel formation for several reasons. First, these stages coincide with the transition of the embryo from the oviduct to the uterus, which is hypothesized to reflect a drop in oxygen tension (Jeremy G Thompson et al., 1996). Oxygen tension of the reproductive tract of mammals ranges from $2-9 \%$, with the uterus often exhibiting a lower tension than the oviduct (Fischer \& Bavister, 1993). Second, the $\mathrm{Na}+\mathrm{K}+$ ATPase pump has to begin working to form the blastocoel cavity, and protein synthesis also begins as the genome is activated, which both require the additional energy provided by glycolysis (Jeremy G 
Thompson et al., 1996). It was determined that the embryo switches to a glycolytic pathway of metabolism because of the production of lactate as a by-product at this stage (Jeremy G Thompson et al., 1996).

During in vitro culture, however, there seems to be a disproportionate increase in the glycolytic rate of metabolism that is linked to lower developmental competence of embryos (H. J. Leese, 2012). In a concept known as the 'quiet embryo hypothesis,' it is theorized that embryos which minimize oxygen consumption from the zygote to the morula stage may lessen the formation of oxygen radicals, limiting damage to cellular and molecular processes occurring during this time, such as cell division and gene expression (Henry J. Leese, 2002). The production of oxygen radicals as a side effect of oxidative phosphorylation inhibits development by causing membrane lipid peroxidation, enzyme inactivation, and DNA damage (Rieger, 1992). If an embryo is more 'noisy,' it signifies a need for greater amounts of energy and nutrients directed toward repair processes (Baumann, Morris, Sreenan, \& Leese, 2007). As a result of this hypothesis, oxygen consumption may be a marker for developmental competence and the culture of embryos in a low $(5 \%)$ oxygen environment rather than an atmospheric $(20 \%)$ environment may promote continued development through the reduction of reactive oxygen species $(H . J$. Leese, 2012; Henry J. Leese, 2002; Rieger, 1992).

The plasticity of pre-implantation embryo metabolism has the benefit of offering leniency when culturing IVP embryos in sub-optimal conditions, but the detriment of making assessment of vital culture components difficult (Absalón-Medina et al., 2014; Gardner et al., 2000). Furthermore, the utilization of any one nutrient by an embryo is dependent on concentrations and ratios of other nutrients present, as well as enzymatic up- or down-regulation in response to this environment (Gardner et al., 2000). Endogenous stores of energy within the embryo come in the form of glycogen and 
triglycerides, however, viability seems to be primarily maintained by exogenous energy substrates (de Souza, Salles, \& Rosa e Silva, 2015).

There are controversial conclusions on the role and potential toxicity of glucose in culture media (Gardner et al., 2000). While glucose is required for nucleic acid production and NADPH synthesis through the pentose phosphate pathway (Absalón-Medina et al., 2014; Gardner et al., 2000), there is also an accumulation of lactate that results from glycolytic activity [Fig. 1] (Gardner et al., 2000). This buildup of lactic acid reduces intracellular $\mathrm{pH}$ and can disturb the function of normal cellular processes such as enzyme activity, cell division, differentiation, and cytoskeletal dynamics (B. Bavister, 1999).

The presence of glucose within culture media may initiate what is known as the crabtree effect, in which glucose inhibits mitochondrial respiration and promotes the less efficient utilization of glycolysis for ATP production (Seshagiri \& Bavister, 1991). The cause of this effect is still relatively unknown, however. It may be that the mechanism for shuttling reducing equivalents across the outer mitochondrial membrane is impaired in in vitro cultured embryos, causing embryos to utilize the glucose present in their surroundings for lactate production to generate $N A D+$, a reducing equivalent (Jeremy $G$ Thompson et al., 1996).

Fructose has been suggested as an alternative energy source for embryos in culture due to its presence in the reproductive tract of the cow (Barceló-Fimbres \& Seidel, 2007a). Fructose results in higher blastocyst yield per oocyte and per 8-cell embryo than glucose, and does not have the putative toxic effects (Barceló-Fimbres \& Seidel, 2007a). When glucose is phosphorylated in the first step of glycolysis, glucose-6-phosphate can fail to inhibit hexokinase activity in the embryo, causing continued phosphorylation of glucose [Fig. 1] (Gardner et al., 2000). The cell withdraws this phosphate from its mitochondrial supply, depleting mitochondrial pools of phosphate and subsequently decreasing ATP production within the mitochondria (Gardner et al., 2000). Because 
fructose is a hexose that can serve as an energy substrate without requiring hexokinase, this may explain the reduction in negative effects seen from substituting some or all of the glucose with fructose.

Studies such as this show that culture media has ample room for improvement as more is learned of embryo metabolism. In addition to the evolution of culture media through improvement of energy substrate provision, the addition of metabolic regulators may aid in regulation of glucose uptake in embryos post-compaction as well as combat some negative artifacts of IVC (Absalón-Medina et al., 2014; Barceló-Fimbres \& Seidel, 2007a). 


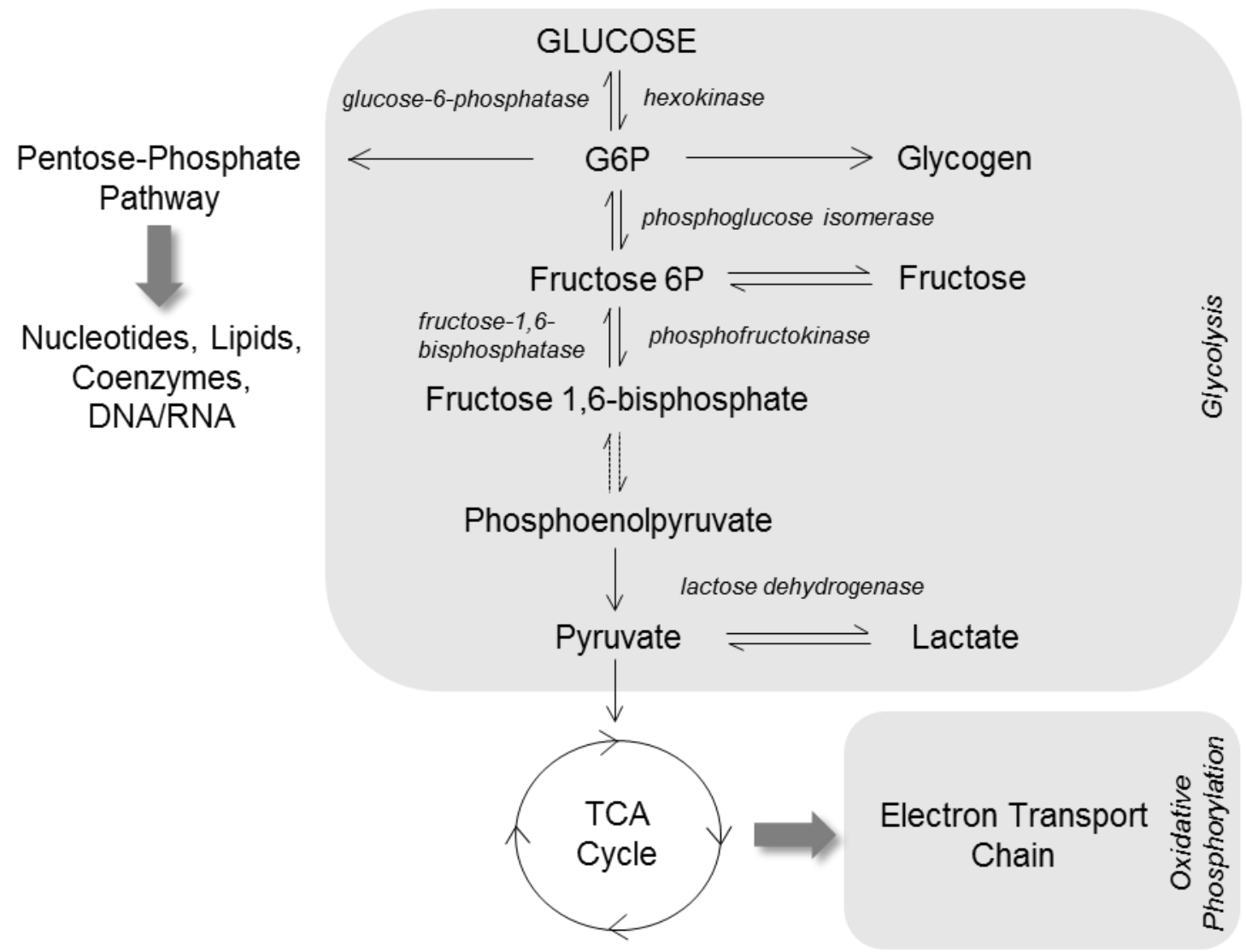

Figure 1. Glucose metabolism within the cell. Glucose is broken down into pyruvate through a series of enzymatic reactions during glycolysis, generating ATP and NADH. Pyruvate is converted to acetyl Co-A where it can then enter the tricarboxylic acid (TCA) cycle to generate $\mathrm{NADH}$ and $\mathrm{FADH}_{2}$, which will go on to donate electrons for oxidative phosphorylation within the electron transport chain, ultimately producing ATP. 


\subsection{Intracellular lipids}

Lipids within the cytoplasm of the oocyte and embryo serve as an endogenous energy source for a variety of biological functions (R. Sturmey, Reis, Leese, \& McEvoy, 2009). Triglycerides within the ooplasm have an important role in energy provision during oocyte maturation, as seen by the sharp reduction in lipid content after this event (E M Ferguson \& Leese, 1999). Because of this role, insufficient lipid content within the ooplasm as indicated by a pale coloration may signify a less viable oocyte due to the lack of necessary substrates for ATP production; this ATP is required for oocyte maturation (Jeong et al., 2009). Lipids are also essential for membrane biosynthesis, hormone synthesis, and cell signaling (Dunning, Russell, \& Robker, 2014; Sutton-McDowall, Feil, Robker, Thompson, \& Dunning, 2012). Cattle embryos have a particularly high lipid content when compared to human and mouse embryos, which is potentially due to the significant expansion that occurs prior to implantation, as compared to the limited expansion seen in the human embryo (Gardner et al., 2000; R. Sturmey et al., 2009). Additionally, in comparison to human and mouse embryos, cattle embryos remain unattached for a longer amount of time in the uterus, thus requiring a larger supply of endogenous energy prior to implantation (R. Sturmey et al., 2009).

Triglycerides are stored within the cell in droplets surrounded by a phospholipid monolayer and lipid droplet coat proteins, which regulate droplet size and access of certain lipases (Dunning et al., 2014). The cells within an embryo utilize triglycerides as an energy source by first cleaving the fatty acids from their glycerol backbone through the action of three sequential lipase enzymes: adipose triglyceride lipase, hormone-sensitive lipase, and monoglyceride lipase (Dunning et al., 2014). These fatty acids are primarily palmitic $(32 \%)$, oleic $(25.1 \%)$, and stearic $(14.2 \%)$, with saturated fatty acids being more abundant (49\%) than mono-(38\%) and poly-unsaturated (14\%) acids (Mcevoy, Coull, Broadbent, Hutchinson, \& Speake, 2000). Fatty acids are then converted to acyl-CoA by fatty acyl 
CoA synthase and transported into the mitochondria through the binding of acyl-CoA to Lcarnitine by carnitine palmitoyl transferase 1B (CPT1B) on the outer mitochondrial membrane [Fig. 2] (Sutton-McDowall et al., 2012). Acyl-carnitine is translocated through the inner mitochondrial membrane by carnitine translocase (CAT), where it is then converted back to acyl-CoA by carnitine palmitoyl transferase 2 (CPTII) on the inner mitochondrial membrane (Sutton-McDowall et al., 2012). Acyl-CoA undergoes $\beta$-oxidation within the mitochondrial matrix to produce acetyl-CoA, while carnitine is shuttled back into the cytoplasm by CAT (Sutton-McDowall et al., 2012). This acetyl-CoA then enters the citric acid cycle and produces precursors for energy capture in the form of ATP (SuttonMcDowall et al., 2012). The rate-limiting step in this process is the action of CPT1B, which is facilitated by the presence of L-carnitine (Sutton-McDowall et al., 2012).

Further evidence for the use of intracellular lipids as an energy source is the rearrangement of mitochondria to be in close proximity to lipid droplets within the oocyte in response to the LH surge (Hyttel, Fair, Callesen, \& Greve, 1997). This provides an easily accessible and potent energy supply to the mitochondria. Glucose metabolism yields between 27 and 31 moles of ATP, while oxidation of palmitate yields around 104 moles of ATP (R. Sturmey et al., 2009). Finally, and perhaps most compellingly, oocytes are able to develop into blastocysts after in vitro fertilization or parthenogenesis when cultured in the complete absence of exogenous energy sources, however, they are not able to develop when triglyceride metabolism is inhibited under the same conditions (Elizabeth $\mathrm{M}$ Ferguson \& Leese, 2006).

It has been shown that impaired embryonic metabolism in an in vitro setting can cause abnormal lipid accumulation due to sub-optimal media composition (BarcelóFimbres \& Seidel, 2007b). Altering embryonic metabolism may provide a way to reduce this lipid accumulation and improve development rates as the embryos are rescued from oxidative stress (Absalón-Medina et al., 2014; Paschoal et al., 2012). These elevated lipid 
levels negatively influence cryotolerance; however, the exact mechanism by which this occurs is unknown (Abe, Yamashita, Satoh, \& Hoshi, 2002; L. Baldoceda, Gagne, Ferreira, \& Robert, 2015; Seidel, 2006). It is possible that lipids influence cryotolerance by lipid peroxidation (Barceló-Fimbres \& Seidel, 2007b), or by changing the composition of cellular membranes by uptake from culture media and integration into existing membranes (Shehab-El-Deen, Leroy, Maes, \& Van Soom, 2009). Lipid accumulation also increases cell volume and may lead to osmotic stress within the embryo (E M Ferguson \& Leese, 1999). Lastly, cryopreservation generates an increased level of oxygen radicals, which may be compounded by the additional oxygen radicals created from an elevated lipid content in IVP embryos, leading to cell death when the two factors are combined (BarcelóFimbres \& Seidel, 2007b).

There are multiple classes of lipids that affect embryo cryotolerance: triacylglycerides (TAG; cytoplasmic lipids), free fatty acids (FFA), cholesterol (membrane lipid), and phospholipids (membrane lipid) (M. Sudano et al., 2013). Of these, TAG seem to be the most influenced by IVC (Abe et al., 2002). TAG may increase in IVP embryos as a result of the Crabtree effect, in which there is a rise in the concentration of lipid synthesis precursors due to an excess of glycolysis and an inhibition of oxidative phosphorylation (Rieger, 1992; M. J. Sudano et al., 2011). Delipation experiments provide evidence for the interference of lipids with cryopreservation, as the removal of lipids through centrifugation increases tolerance to cryopreservation (Ushijima, Yamakawa, \& Nagashima, 1999). Studies such as this suggest that delipation could be an effective technique for improving cryosurvival of bovine embryos (R. Sturmey et al., 2009). This concept has translated into the study of chemical delipation through the addition of metabolic regulators that promote lipolysis to embryo culture medium (M. Sudano et al., 2013). 


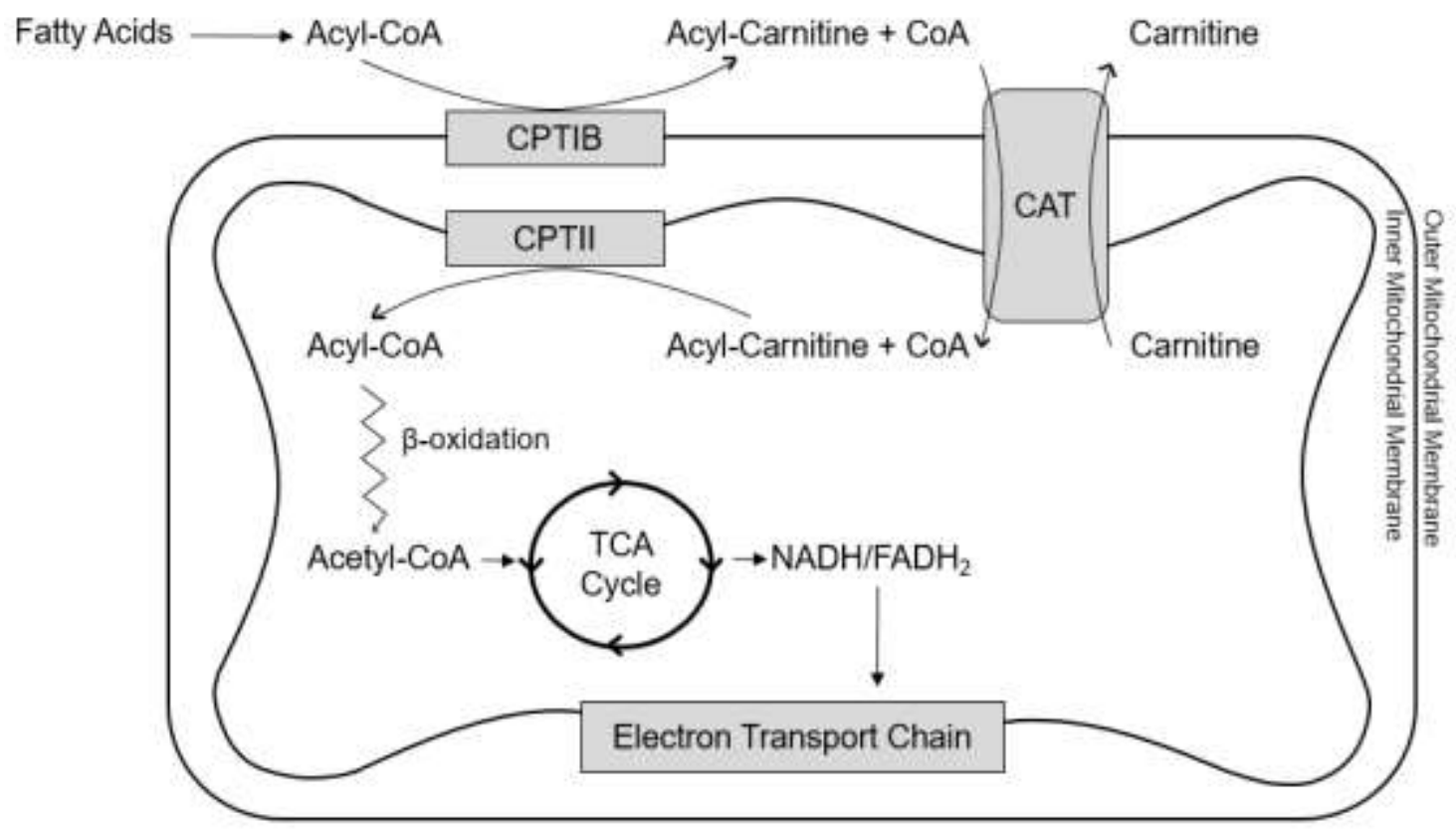

Figure 2. Fatty acid transport into the mitochondria and metabolic pathway. Fatty acids are converted to Acyl-CoA, then bound to carnitine by carnitine palmitoyl transferase 1B (CPT1B). Acyl-Carnitine $+\mathrm{CoA}$ is transported through the outer and inner mitochondrial membranes by carnitine translocase (CAT). Carnitine is removed and AcylCoA is regenerated by carnitine palmitoyl transferase 2 (CPTII). Acyl-CoA then undergoes $\beta$-oxidation to generate Acetyl-CoA, which enters the tricarboxylic acid (TCA) cycle to generate electron donors for the electron transport chain. 


\subsection{Mitochondrial function}

Mitochondria are maternally inherited organelles responsible for ATP production through oxidative phosphorylation and originate from a defined founder population within the oocyte (Cummins, 2004; Dumollard, Duchen, \& Carroll, 2007). The importance of oxidative phosphorylation as an energy source for maturing oocytes and developing embryos has given rise to the study of mitochondrial activity as an indicator of developmental competence (Tarazona, Rodríguez, Restrepo, \& Olivera-Angel, 2006). Mitochondria may regulate development of the embryo through modulation of Calcium signaling, generation of ATP, maintenance of intracellular redox potential, and initiation of apoptosis (Dumollard et al., 2007). Mitochondria produce both reactive oxygen species (ROS) and reducing equivalents (NAD $(P) H / N A D(P)+)$, a balance of which is crucial for

proper development (Dumollard, Carroll, Duchen, Campbell, \& Swann, 2009; Dumollard et al., 2007).

The oocyte and early embryo up to the eight cell stage contain immature, ovoidshaped mitochondria with peripheral and underdeveloped cristae (Plante \& King, 1994). It is controversial whether these immature mitochondria are efficient energy producers. While it would seem that the immature structure of the mitochondria would hinder energy derivation potential, mitochondrial activity increases during oocyte maturation for the continuation of events such as nuclear maturation, cytoplasmic maturation, cytoskeletal rearrangement, and mRNA accumulation (Tarazona et al., 2006). Between the 8-cell and morula stage, mitochondria mature and become elongated with well-developed transverse cristae (Houghton \& Leese, 2004; Plante \& King, 1994). Although this maturation indicates a more competent mitochondrion, early embryonic divisions up to the 8 cell stage are marked by low to intermediate mitochondrial activity, and genome activation signifies a decrease to very low mitochondrial activity (Tarazona et al., 2006). This may be because 
genome activation coincides with a switch to alternate energy derivation pathways, such as glycolysis (Jeremy G Thompson et al., 1996).

Pre-implantation embryos are particularly sensitive to oxidative stress (Dumollard et al., 2009). If this oxidative stress does not lead to cellular apoptosis or developmental arrest, it may impair mitochondrial function by causing a redox imbalance and interfere with the embryo's ability to properly utilize energy substrates, creating a buildup of intracellular lipids and inferior embryo quality (Gad et al., 2012). Mitochondrial dysfunction can also affect vital processes such as spindle organization, chromosomal segregation, timing of the cell cycle, compaction, cavitation, and blastocyst hatching (Van Blerkom, 2004). These abnormalities highlight the important balance of antioxidants and ROS maintained by the mitochondria (Dumollard et al., 2009, 2007; Gad et al., 2012). Typically, bovine embryos in an in vitro setting accumulate an excess of ROS that exceed the antioxidant capacity that the embryo naturally generates through metabolic intermediates and reducing equivalents from the TCA cycle (Dumollard et al., 2009). As a result, much effort has been focused on optimizing the redox state of the embryo. This was initially done by reducing the oxygen tension of the culture environment from $20 \%$ to $5 \% \mathrm{O} 2$, however, these conditions do not completely remedy the increased ROS (Rieger, 1992). The addition of antioxidants to culture medium has since become a prominent approach to address this issue (Fakruzzaman et al., 2015). 


\subsection{Metabolic regulators}

When considering an alteration to embryo culture medium, there are multiple factors that must be taken into account. The addition of an exogenous chemical to culture medium may have detrimental downstream effects depending on the stage of development that the embryo is in and the pathway of action of the substance. It is important to consider whether the chemical or molecule will interfere with major life events of the embryo, such as genome activation, mitochondrial maturation, and/or compaction (Gad et al., 2012).

\subsubsection{L-Carnitine}

L-carnitine facilitates the rate-limiting step of fatty acid transport into the mitochondria [Fig. 2] (Sutton-McDowall et al., 2012). For this reason, this substance has been looked to as a potential regulator of fatty acid metabolism (M. Sudano et al., 2013). Addition of L-carnitine to IVC medium has been shown to decrease intracellular lipid accumulation, improve blastocyst development rate, and decrease ROS in bovine embryos (L. Baldoceda et al., 2015; T. Takahashi et al., 2013). This is a peculiar occurrence because the up-regulation of fatty acid metabolism and increased metabolic rate would imply an increase in ROS (Takahashi et al., 2013). However, L-carnitine seems to serve as an antioxidant by scavenging free radicals, resulting in a net decrease of ROS within the embryo (Baldoceda et al., 2015; Takahashi et al., 2013). Consequently, because of the correlation between intracellular lipid content and cryotolerance (Abe et al., 2002), the reduction of lipids by L-carnitine offers a non-invasive way to improve freezing survival of bovine embryos (L. Baldoceda et al., 2015; T. Takahashi et al., 2013).

\subsubsection{Forskolin}

Forskolin is a diterpene molecule that was discovered to activate adenylate cyclase on the membranes of cells (Seamon, Padgett, \& Daly, 1981). Forskolin is unique in that it acts in a reversible manner on the cAMP-generating systems within a cell 
(Seamon et al., 1981). An increased level of intracellular cAMP initiates a release of the catalytic subunits of protein kinase $A(P K A)$ from the regulatory subunits, which in turn activate endogenous lipase enzymes, such as hormone sensitive lipase, through phosphorylation [Fig. 3] (Londos et al., 1999; Men, Agca, Riley, \& Critser, 2006). These lipase enzymes go on to hydrolyze TAG into fatty acids and glycerol, freeing the fatty acids to undergo $\beta$-oxidation (Men et al., 2006). Forskolin was first studied as a culture medium additive in porcine embryos and found to significantly reduce cytoplasmic lipids (Men et al., 2006). Subsequent experiments in bovine IVC have shown that addition of forskolin to culture medium improves cryosurvival without negative effects on embryo development (Paschoal et al., 2012; Sanches et al., 2013). 


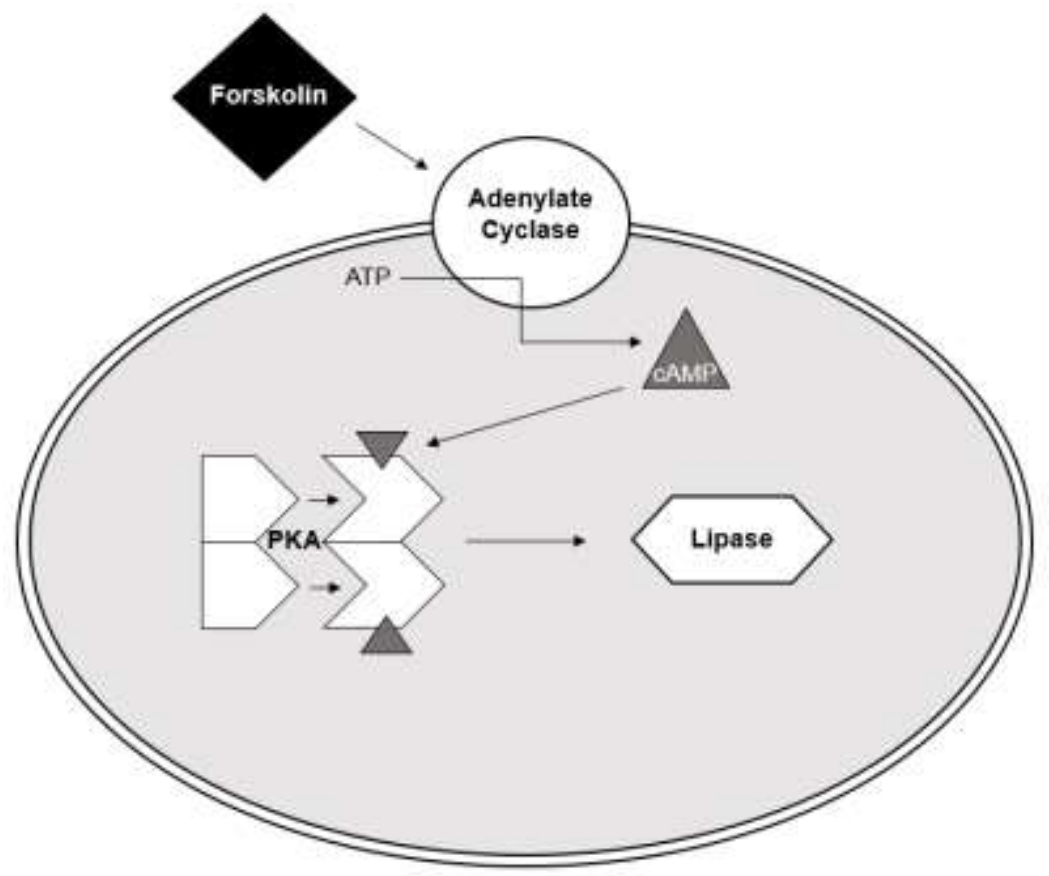

Figure 3. Lipolytic pathway of forskolin within the cell. Forskolin activates adenylate cyclase on the membranes of cells, which increases intracellular cyclic adenosine monophosphate (cAMP). cAMP then binds to the catalytic subunits of phosphokinase A (PKA), which goes on to activate the lipase enzymes involved in breaking down triacylglycerol into free fatty acids that can be metabolized for ATP synthesis. 


\subsubsection{Vitamin $K_{2}$}

It was recently found that vitamin $\mathrm{K}_{2}$ is able to restore mitochondrial function in a strain of Drosophilia engineered with a genetic mutation affecting their mitochondria (Vos et al., 2012). From this study it was concluded that vitamin $\mathrm{K}_{2}$ serves as an electron carrier in eukaryotic cells and affects mitochondrial remodeling through the restoration of efficient ATP production [Fig. 4] (Vos et al., 2012). Ovoid and immature mitochondria signify a deficiency in energy utilization (Vos et al., 2012) and developmental incompetence (Van Blerkom, 2009). This trend is often observed in IVP bovine embryos, indicating there is mitochondrial dysfunction in these cells as a result of culture conditions (Abe et al., 2002). It was initially believed that mitochondrial dysfunction was a result of oxidative stress from the production of free radicals, but the failure of antioxidant culture additives to restore mitochondrial function may signify an ineptitude of mitochondria in IVP embryos (Baldoceda-Baldeon, Gagne, Vigneault, Blondin, \& Robert, 2014). A recent study has

shown that vitamin $\mathrm{K}_{2}$ as a culture media additive improves bovine embryo phenotype, and has the potential to increase blastocyst development rates by rescuing embryos that would have otherwise ceased development (Baldoceda-Baldeon et al., 2014). 


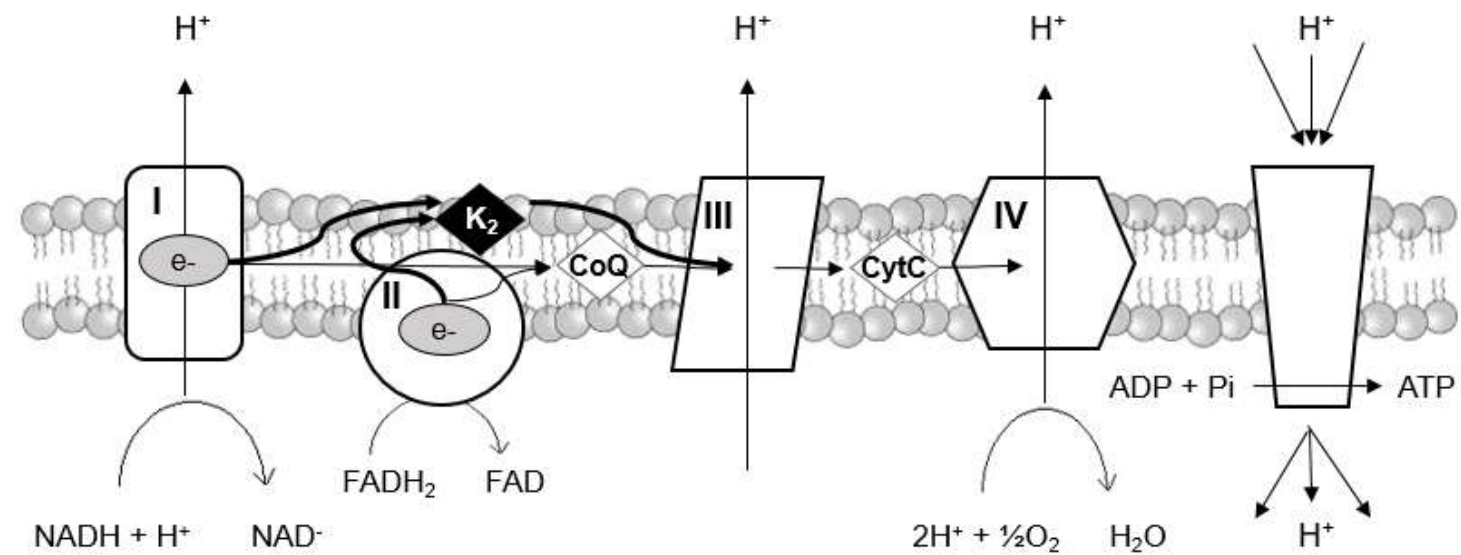

Figure 4. Proposed mechanism of vitamin $\mathrm{K}_{2}$ within the electron transport chain. Vitamin $\mathrm{K}_{2}$ receives electrons from complexes I and II and transports the electrons to complex III. This prevents premature loss of electrons from the transport chain and reduces the generation of reactive oxygen species by the conversion of oxygen to water. 


\subsubsection{Coenzyme $Q_{10}$}

Similar to vitamin $K_{2}$, it is theorized that supplementation of coenzyme $Q_{10}$ to embryo culture medium may improve development rates by restoring disturbed function of the electron transport chain (Stojkovic et al., 1999). Coenzyme $Q_{10}$ is an electron carrier that resides in the intermembrane space of the inner mitochondrial membrane bilayer, transporting electrons from complexes I and II to complex III [Fig. 4] (Stojkovic et al., 1999). Vitamin $K_{2}$ and coenzyme $Q_{10}$ are both lipophilic and insoluble, making the addition of these molecules to water-based culture medium a challenge. Coenzyme $Q_{10}$ is made soluble through a method of creating a submicron-sized dispersion within a stabilizing solvent (Stojkovic et al., 1999). It has been shown that the addition of this solution to culture medium has positive effects on blastocyst formation, cell proliferation, hatching, and ATP content of bovine embryos, possibly due to a restoration of proper electron transport chain function (Stojkovic et al., 1999).

\subsubsection{Conditioned media}

The prolonged exposure of IVC embryos to imperfect culture conditions has given rise to developmental abnormalities and a blastocyst rate that remains relatively low (Chen et al., 1994). A proposed remedy for the shortcomings of embryo culture medium is a coculture system in which a population of feeder cells supplies specific growth factors to the medium in which the embryos develop (Chen et al., 1994). This technique originates from the theory that embryos cultured in vitro lack the cell to cell interaction that is present in vivo, thereby lacking exposure to specific hormones, growth factors, and cell signaling factors that the niche environment provides (Maeda, Kotsuji, Negami, Kamitani, \& Tominaga, 1996). Modifications of this co-culture system now include extracting the media used to nourish a cell monolayer and re-distributing it to the media of another culture system (Chen et al., 1994). 
Vero cells, derived from kidney cells of the Green African monkey, have been used as a source for co-culture serum extraction because they are inexpensive, easy to handle, and pathogen-free as a result of their use in vaccine-production (Chen et al., 1994). Specifically, these cells produce interleukin, platelet-derived growth factor, Leukemia inhibitory factor, and insulin-like growth factor, which are considered embryotrophic because of their stimulatory effect on embryonic development (Tavares et al., 2011). In studies performed with mouse embryos, co-culture with Vero cells themselves as well as with peptides extracted from the cellular monolayer resulted in rescue from the developmental block usually encountered before the blastocyst stage (Chen et al., 1994). Later studies with bovine embryos showed that the proteins, cell signaling molecules, and growth factors found in this cellular monolayer serum had similar embryotrophic effects (Maeda et al., 1996). 


\subsection{Summary}

To date, many deficiencies still reside in the process for commercial production of embryos in vitro. Research into the mechanisms of developmental control of bovine embryos has allowed for moderate improvements in development rates, but progress is still needed. What is known is that dysfunction in energy metabolism results in abnormal accumulation of intracellular lipids, causing decreased cryotolerance (Barceló-Fimbres \& Seidel, 2007b). The mostly likely sources of this dysfunction are inadequacies in culture media (Barceló-Fimbres \& Seidel, 2007b). Research focusing on the rescue of embryos from the detrimental effects of an in vitro environment through enhancement of culture media composition is a promising approach toward improving the quality, cryotolerance, and overall competence of bovine IVP embryos. 


\section{Chapter 2. Common Materials and Methods}

\subsection{Materials}

All materials used for in vitro embryo production were obtained from MOFA Global, LLC, Verona, WI, USA and all chemicals were obtained from Sigma-Aldrich, St. Louis, MO, USA unless otherwise stated.

\subsection{In vitro embryo production}

Embryos for each experiment and pilot study were produced by the following methods with modifications as described in respective chapters.

\subsubsection{Oocyte collection}

Ovaries were acquired from a local abattoir and transported to the laboratory at ambient temperature $\left(22^{\circ} \mathrm{C}-25^{\circ} \mathrm{C}\right.$ ) in $0.9 \% \mathrm{NaCl}$ saline solution (Abbott Laboratories, Abbott Park, IL, USA) within $3 \mathrm{~h}$ of slaughter. Oocytes were aspirated from 2 to $8 \mathrm{~mm}$ follicles using an 18-ga needle attached to a vacuum pump with 25 to $30 \mathrm{~mm} \mathrm{Hg}$ of pressure. Oocytes were held in OPU medium containing $0.3 \%$ BSA and $50 \mu \mathrm{g} / \mathrm{mL}$ heparin (19982/1281; MOFA Global) during aspiration then washed three times in oocyte wash medium containing 0.3\% BSA (19982/1200; MOFA Global) and $2.5 \mu \mathrm{g} / \mathrm{mL}$ gentamicin (Sigma-Aldrich). Immature oocytes containing at least three layers of compact cumulus cells were selected for IVM.

\subsubsection{In vitro maturation}

Cumulus-oocyte-complexes (COCs) were matured in $\mathrm{CO}_{2}$-gassed bicarbonatebased medium designed for using a conventional maturation system. Groups of approximately 40 COCs were placed into $0.8 \mathrm{~mL}$ tissue culture medium (TCM) -199 supplemented with $10 \%$ fetal calf serum (v:v; Thermo Fisher Scientific, Waltham, MA, USA), $0.4 \mathrm{mM}$ pyruvate, 5 IU bovine FSH and LH, and $0.625 \mu \mathrm{g} / \mathrm{mL}$ gentamicin (SigmaAldrich). COCs were incubated at $38.5^{\circ} \mathrm{C}$ in $5 \% \mathrm{CO}_{2}$ humidified air for $23 \mathrm{~h}$. 


\subsubsection{In vitro fertilization}

Expanded COCs were transferred in groups of 50 to $0.45 \mathrm{~mL}$ pre-equilibrated fertilization chemically defined fertilization medium (FCDM) containing 0.5\% BSA, 2.0 $\mu \mathrm{g} / \mathrm{mL}$ heparin (H-3125; Sigma) and 5 mM caffeine (9982/1266; MOFA Global). Semen from bulls ( $n=2$ to 3 ) with proven fertility was processed through a 45 and $90 \%$ Percoll gradient and centrifuged at $400 \times \mathrm{g}$ at $23^{\circ} \mathrm{C}$ for $12 \mathrm{~min}$. The pellet was removed and washed in $1 \mathrm{~mL} F C D M$, then centrifuged again at $400 \times \mathrm{g}$ at $23^{\circ} \mathrm{C}$ for 4 minutes. The supernatant from the final wash was removed and the concentration of sperm cells in the resulting pellet was measured using a hemocytometer (Bright-Line; Hausser Scientific, Horsham, PA, USA) and inverted microscope (Olympus IX71; Center Valley, PA, USA). The pellet was diluted to a final concentration of $5 \times 10^{5} \mathrm{sperm} / \mathrm{mL}$ using FCDM. Coincubation in a final volume of $0.5 \mathrm{~mL}$ took place at $38.5^{\circ} \mathrm{C}$ in $5 \% \mathrm{CO}_{2}$ humidified air for 20 h.

\subsubsection{Embryo culture}

Presumptive zygotes were removed from culture dishes in groups of 150 and placed into a $0.5 \mathrm{~mL}$ Eppendorf tube with $50 \mathrm{uL}$ oocyte washing medium. Tubes were vortexed at maximum speed for 2 min to remove cumulus cells and presumptive zygotes were washed through drops of oocyte medium. Presumptive zygotes were placed into preequilibrated defined embryo culture media (BBH7 (MOFA Global or SOF based media (SCF1, SOF for Conventional Freezing 1)) drops at a ratio of 1.5 to $2.0 \mathrm{uL}$ media per

zygote, covered with mineral oil (Sigma-Aldrich), and cultured at $38.5^{\circ} \mathrm{C}$ in $5 \% \mathrm{CO}_{2}, 5 \%$ $\mathrm{O}_{2}$ and $90 \% \mathrm{~N}_{2}$ (v:v) for 7 to 9 days. 


\subsection{Evaluation of embryos}

The following evaluative methods were used to assess embryos in each pilot study and experiment with modifications as described in respective chapters.

\subsubsection{Cleavage and development}

Cleavage and 5 to 8 cell development were assessed using a stereomicroscope 2.5 days after fertilization. Blastocyst rate was evaluated at 7,8 , and 9 days after fertilization and calculated as a percentage of total oocytes placed into culture. Stage (5: early blastocyst, 6: blastocyst, 7: expanded blastocyst, 8: hatched blastocyst) and grade (1: excellent, 2: fair, 3: poor, 4: dead) of blastocysts were recorded at days 7 and 8 according to the guidelines of the International Embryo Transfer Society (Robertson \& Nelson, 1998).

\subsubsection{Lipid content}

To quantify lipid accumulation, stage 7 blastocysts of grades 1 or 2 were fixed in $10 \%$ paraformaldehyde (v:v) for at least $24 \mathrm{~h}$. A $1 \mathrm{mg} / \mathrm{mL}$ stock solution of Nile Red dye (Sigma-Aldrich) was prepared in DMSO and stored at room temperature in the dark. Fixed embryos were washed in three drops of PBS-PVP then stained in a $1 \mu \mathrm{g} / \mathrm{mL}$ solution of Nile Red dye in PBS-PVP for 30 min. Embryos were washed again with PBS-PVP and mounted in a 1:10 (v:v) dilution of ProLong Diamond Antifade Mountant (ThermoFisher Scientific, Waltham, MA, USA) in PBS. Images were acquired at an excitation/emission wavelength of $488 / 520$ (imaging technique described in respective chapters). As a negative control, embryos were placed in $99 \%$ ethanol for $18 \mathrm{~h}$ after fixation to dissolve intracellular lipids then stained and imaged as previously described.

\subsubsection{Mitochondrial polarity}

Mitochondrial polarity was assessed by staining stage 7 blastocysts of grades 1 or 2 with an active mitochondrial dye that binds differentially based on mitochondrial membrane potential. Live embryos were cultured in SCF1 medium containing $300 \mathrm{nM}$ 
MitoTracker Red CMX-rosamine (Molecular Probes, Eugene, OR, USA) for $45 \mathrm{~min}$ at $38.5^{\circ} \mathrm{C}$ in $5 \% \mathrm{CO}_{2}$ humidified air. As a negative control, a group of live embryos were randomly selected to be cultured in $100 \mathrm{nM}$ carbonyl cyanide m-chlorophenylhydrazone (CCCP), a mitochondrial membrane uncoupler, in addition to MitoTracker Red CMXrosamine. Embryos were washed in three drops of PBS-PVP and fixed in $10 \%$ paraformaldehyde (v:v) for $24 \mathrm{~h}$. Embryos were then stained with $1 \mu \mathrm{g} / \mathrm{mL}$ DAPI for 10 min, washed with PBS-PVP, and mounted in a 1:10 (v:v) dilution of ProLong Diamond Antifade Mountant (ThermoFisher Scientific, Waltham, MA, USA) in PBS for imaging. Imaging technique is explained in respective chapters. 


\section{Chapter 3. Pilot Study 1: Coenzyme $Q_{10}$ Titration}

\subsection{Introduction}

Coenzyme $\mathrm{Q}_{10}(\mathrm{CoQ})$ is a lipophilic antioxidant that is synthesized endogenously and found in the membranes of cells. There are three main functional roles of CoQ. First, in its fully oxidized ubiquinone form, it serves as an electron transporter from complexes I and II to complex III in the mitochondrial electron transport chain (Bentinger, Tekle, \& Dallner, 2010). Second, in its fully reduced ubiquinol form, CoQ acts as an antioxidant in the inner mitochondrial membrane and inhibits lipid peroxidation by scavenging free radicals (Kwong et al., 2002). Third, in its partially reduced ubisemiquinone form, CoQ serves as a source of superoxide and hydrogen peroxide generation (Boveris, Cadenas, \& Stoppani, 1976; Turrens, 1997). Thus, CoQ can act as both a pro-oxidant and an antioxidant, but its primary role is as an antioxidant (Kwong et al., 2002).

It is believed that the increased oxidative stress in IVP embryos is a result of mitochondrial dysfunction, which causes a decrease in developmental competence (Gad et al., 2012). Impaired mitochondrial function also impedes development by causing a buildup of intracellular lipids due to improper utilization of energy substrates (Gad et al., 2012). Particularly in an in vitro setting, bovine embryos seem to accumulate ROS that exceed their natural antioxidant capacity (Dumollard et al., 2009). Because of these negative effects of in vitro culture, research has focused on regaining a balance in redox state as a way to improve the quality of bovine IVP embryos, as measured by the percentage of oocytes that reach the blastocyst stage.

While some studies have focused on the use of antioxidants to counter the accumulation of ROS (Fakruzzaman et al., 2015), there is an alternate approach to addressing this mitochondrial dysfunction. The addition of exogenous CoQ has shown

promising benefit to IVP bovine embryo development (Stojkovic et al., 1999). When comparing several concentrations of $\mathrm{CoQ}(10 \mu \mathrm{M}, 30 \mu \mathrm{M}$, and $100 \mu \mathrm{M})$ in a solubilized 
submicron-sized dispersion, Stojkovic and associates have shown that a concentration of $30 \mu \mathrm{M}$ increased the percentage of blastocysts at 186 hours post insemination (hpi) and the percentage of hatched blastocysts $210 \mathrm{hpi}$ when compared to the other concentrations and a control (Stojkovic et al., 1999). Additionally, in this study, the supplementation of culture media with $30 \mu \mathrm{M} \mathrm{CoQ}$ increased the total cell number of resulting blastocysts when compared to a negative control (Stojkovic et al., 1999).

The objective of the present pilot study was to compare the effects of 10, 30, 100, and $300 \mu \mathrm{M} \mathrm{CoQ}$ within the given culture system. The concentration found to be most effective in improving embryo quality can then be used for subsequent experiments to determine the efficacy of $\mathrm{CoQ}$ in comparison to other metabolic regulators. Based on previous studies (Stojkovic et al., 1999), it was hypothesized that embryos cultured with $30 \mu \mathrm{M} \mathrm{CoQ}$ would show an increase in blastocyst rate, a decrease in lipid content, and an increase in polarity of the inner mitochondrial membrane. 


\subsection{Materials and methods}

3.2.1 In vitro embryo production

Embryos were produced from oocytes $(n=1252)$ as described in Chapter 2.2 with the following modifications.

\subsubsection{Addition of coenzyme $Q_{10}$}

A $0.115 \mathrm{M}$ stock of $\mathrm{CoQ}$ (Homogenous liposomal ubiquinol, Tishcon Corp, Westbury, NY, USA) was diluted to a $1.5 \mathrm{mM}$ intermediate solution using chemically defined culture medium. At day 3 after fertilization, culture drops containing 20-25 fertilized oocytes were randomly assigned to receive one of five CoQ concentrations: $0 \mu \mathrm{M}, 10 \mu \mathrm{M}$, $30 \mu \mathrm{M}, 100 \mu \mathrm{M}$, or $300 \mu \mathrm{M}$. Embryos were then allowed to culture in their respective treatments until day 8.

\subsubsection{Evaluation of embryos}

Cleavage and development, lipid content, and mitochondrial polarity of embryos was evaluated as described in Chapter 2.3 with the following specifications.

\subsubsection{Embryo development}

Percentage of oocytes that cleaved and reached the 5-8 cell stage was recorded but not used for analysis in this study due to treatment beginning at day 3. Percentage of oocytes reaching the blastocyst stage was recorded and analyzed for each treatment group.

\subsubsection{Lipid content}

Lipid accumulation was quantified by staining stage 7 blastocysts with the lipophilic Nile Red dye as described in Chapter 2.3.2. Images were acquired by confocal microscopy at 40X magnification using an excitation/emission wavelength of 488/520 (Olympus Fluoview, Tokyo, Japan). Ten orthogonal projections along the equatorial section of the embryo were collected with a $5 \mu \mathrm{m}$ step size. Fluorescence was measured using Image J software (National Institutes of Health, Bethesda, MD, USA) with background fluorescence 
corrected for, and mean fluorescence between treatment groups was analyzed. Results are expressed in mean fluorescence arbitrary units (AUs).

\subsubsection{Mitochondrial polarity}

Mitochondrial polarity was assessed using MitoTracker Red CMX-rosamine dye, which binds with varying affinity based on degree of mitochondrial polarity, as described in Chapter 2.3.3. Images were acquired by confocal microscopy at $40 \mathrm{X}$ magnification using an excitation/emission wavelength of 594/608 (Olympus Fluoview, Tokyo, Japan). Ten orthogonal projections along the equatorial section of the embryo were collected with a $5 \mu \mathrm{m}$ step size. Fluorescence intensity was measured using ImageJ software (National Institutes of Health, Bethesda, MD, USA) with background fluorescence corrected for, and mean fluorescence between treatment groups was analyzed. Results are expressed in mean fluorescence intensity arbitrary units (AUs).

\subsubsection{Experimental design}

This experiment was designed as a titration of varying concentrations of CoQ: 0 $\mu \mathrm{M}$ (control), $10 \mu \mathrm{M}, 30 \mu \mathrm{M}, 100 \mu \mathrm{M}$, and $300 \mu \mathrm{M}$. Embryos were randomly assigned to one of five treatment groups at 72 hpi. Blastocyst rate, lipid content, and mitochondrial polarity were recorded for each treatment group during each trial replicate $(n=5)$.

\subsubsection{Statistical analysis}

Blastocyst rate, lipid content, and mitochondrial polarity were evaluated by one way-ANOVA using the SAS statistical software package general linear model (GLM; SAS Institute, Cary, NC, USA). If the ANOVA was significant $(P<0.05)$, means were separated by Tukey's HSD. 


\subsection{Results}

\subsubsection{Blastocyst rate}

The blastocyst rate per oocyte was no different $(P>0.05)$ among the control group and each concentration of $\mathrm{CoQ}(11.3 \%$ Control vs. $8.9 \%$ in $10 \mu \mathrm{M}$ CoQ, $10.5 \%$ in $30 \mu \mathrm{M}$ CoQ, $10.6 \%$ in $100 \mu \mathrm{M} \mathrm{CoQ}$, and $7.0 \%$ in $300 \mu \mathrm{M}$ CoQ) [Fig. 5].

\subsubsection{Lipid content}

Supplementing embryo culture media with $10 \mu \mathrm{M}$ and $30 \mu \mathrm{M}$ resulted in a decrease $(\mathrm{P}<0.05)$ in lipid content as compared to the control group (222.3 AFUs and 248.7 AFUs vs. 330.1 AFUs, respectively). No difference ( $P>0.05)$ was seen between the $100 \mu \mathrm{M}$ or $300 \mu \mathrm{M}$ treatment group and the control (262.2 AFUs and 350.2 AFUs vs. $330.1 \mathrm{AFUs,}$ respectively) [Fig. 6].

\subsubsection{Mitochondrial polarity}

No difference $(P>0.05)$ was seen between embryos cultured in the presence of 30 $\mu \mathrm{M} \mathrm{CoQ}$ and the control group (7904.3 AFUs vs. 8001.2 AFUs, respectively). However, embryos cultured in the presence of $10 \mu \mathrm{M}, 100 \mu \mathrm{M}$, and $300 \mu \mathrm{M}$ CoQ each had a decrease $(P<0.05)$ in mitochondrial polarity as compared to the control $(6788.0$ AFUS, 5713.7 AFUs, and 2112.8 AFUs vs. 8001.2 AFUs, respectively). Furthermore, the $10 \mu \mathrm{M}$, $100 \mu \mathrm{M}$, and $300 \mu \mathrm{M}$ treatments groups all differed $(P<0.05)$ from one another, with mitochondrial polarity decreasing as the concentration of CoQ increased [Fig. 7]. 


\section{Blastocyst Rate}

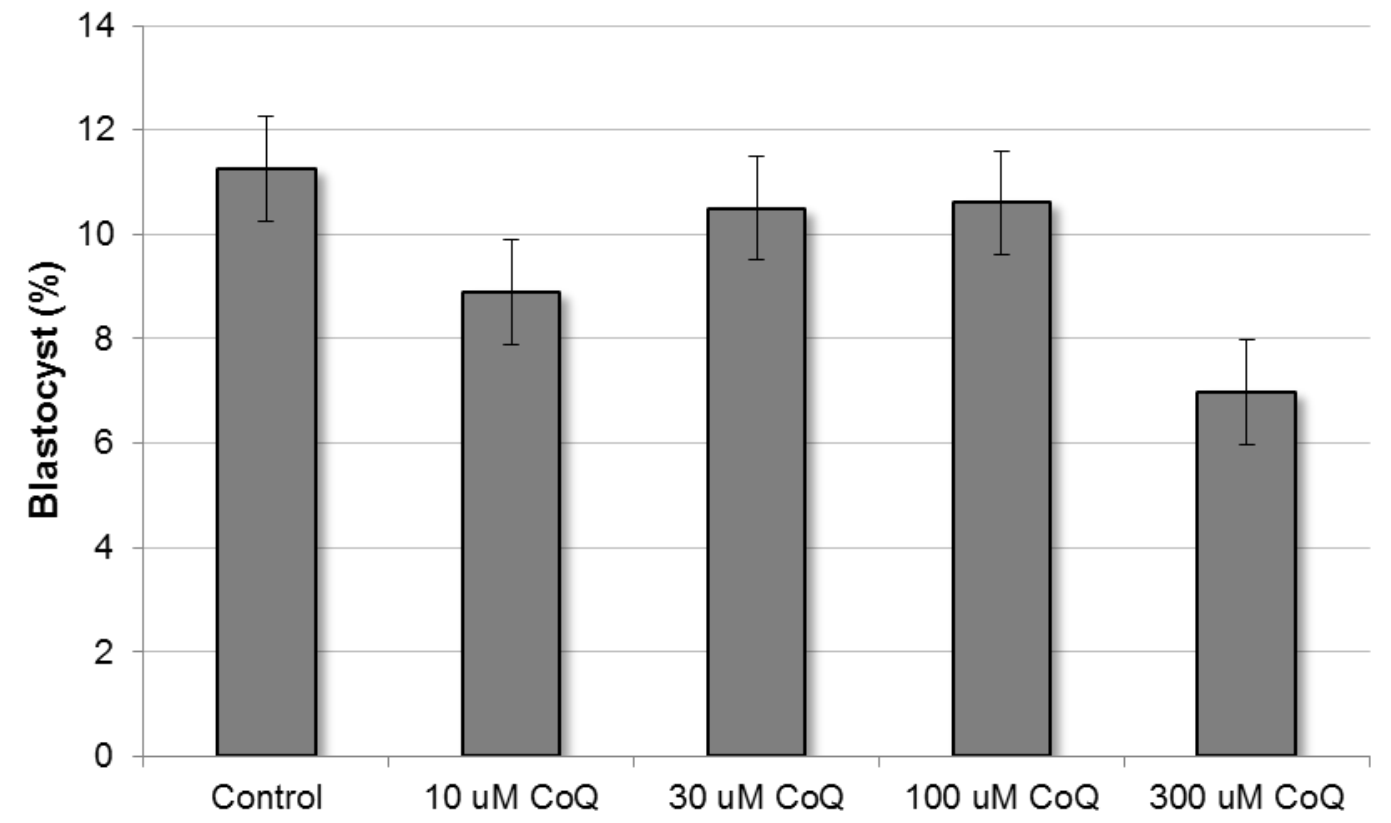

Figure 5. Blastocyst rate between CoQ concentrations. Blastocyst development rates between treatment groups did not differ $(P>0.05)$. Error bars represent standard error of the mean. $\mathrm{N}=5$ replicates. 


\section{Lipid Content}

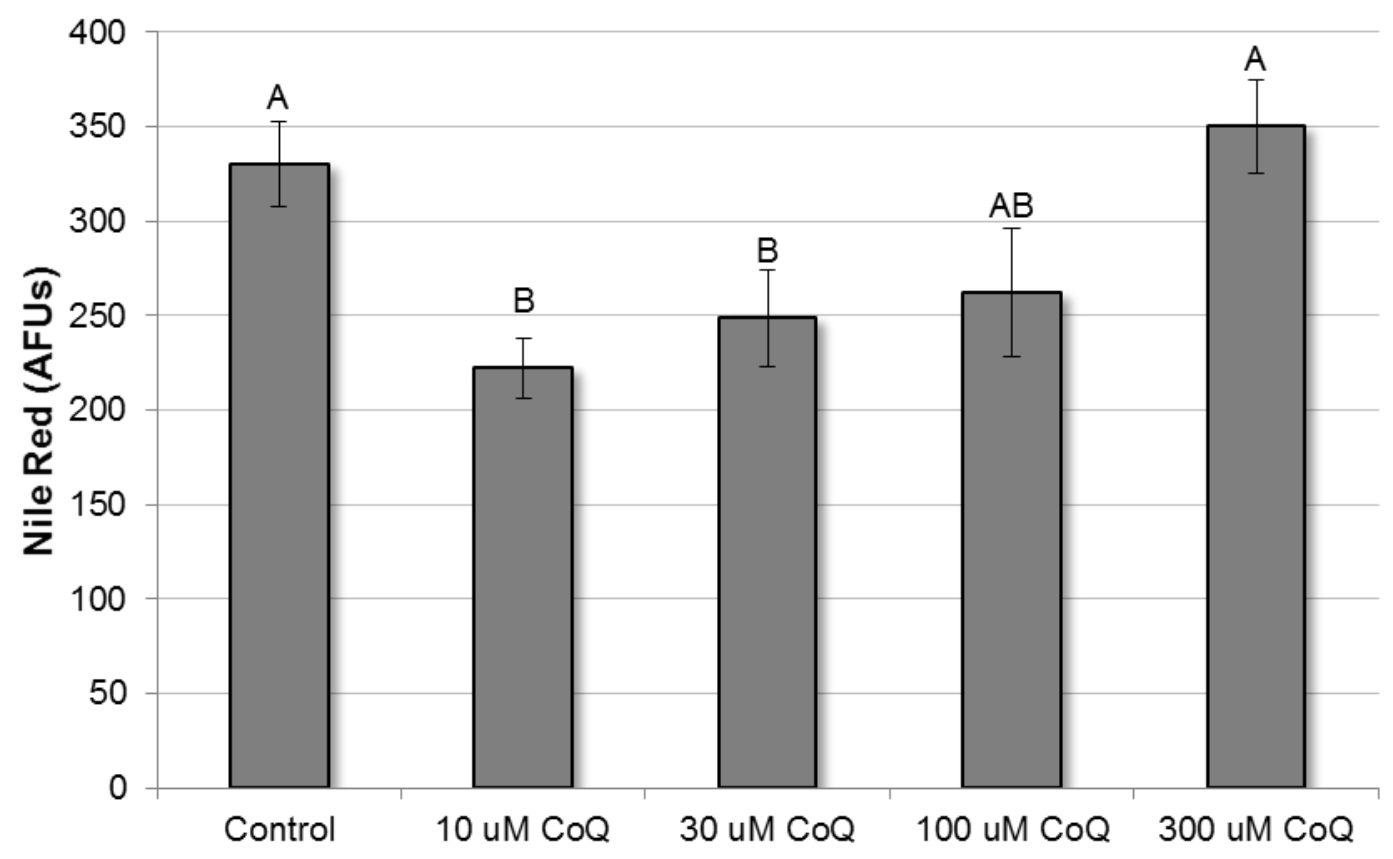

Figure 6. Lipid content between CoQ concentrations. Means of lipid content between treatment groups as represented by Nile Red AFUs (Control ( $n=50), 10 \mu \mathrm{M} \operatorname{CoQ}(n=78)$, $30 \mu \mathrm{M} \mathrm{CoQ}(\mathrm{n}=40), 100 \mu \mathrm{M} \mathrm{CoQ}(\mathrm{n}=20)$, and $300 \mu \mathrm{M} \mathrm{CoQ}(\mathrm{n}=40))$. Error bars represent standard error of the mean, and values without common labels differ $(P<0.05)$. 


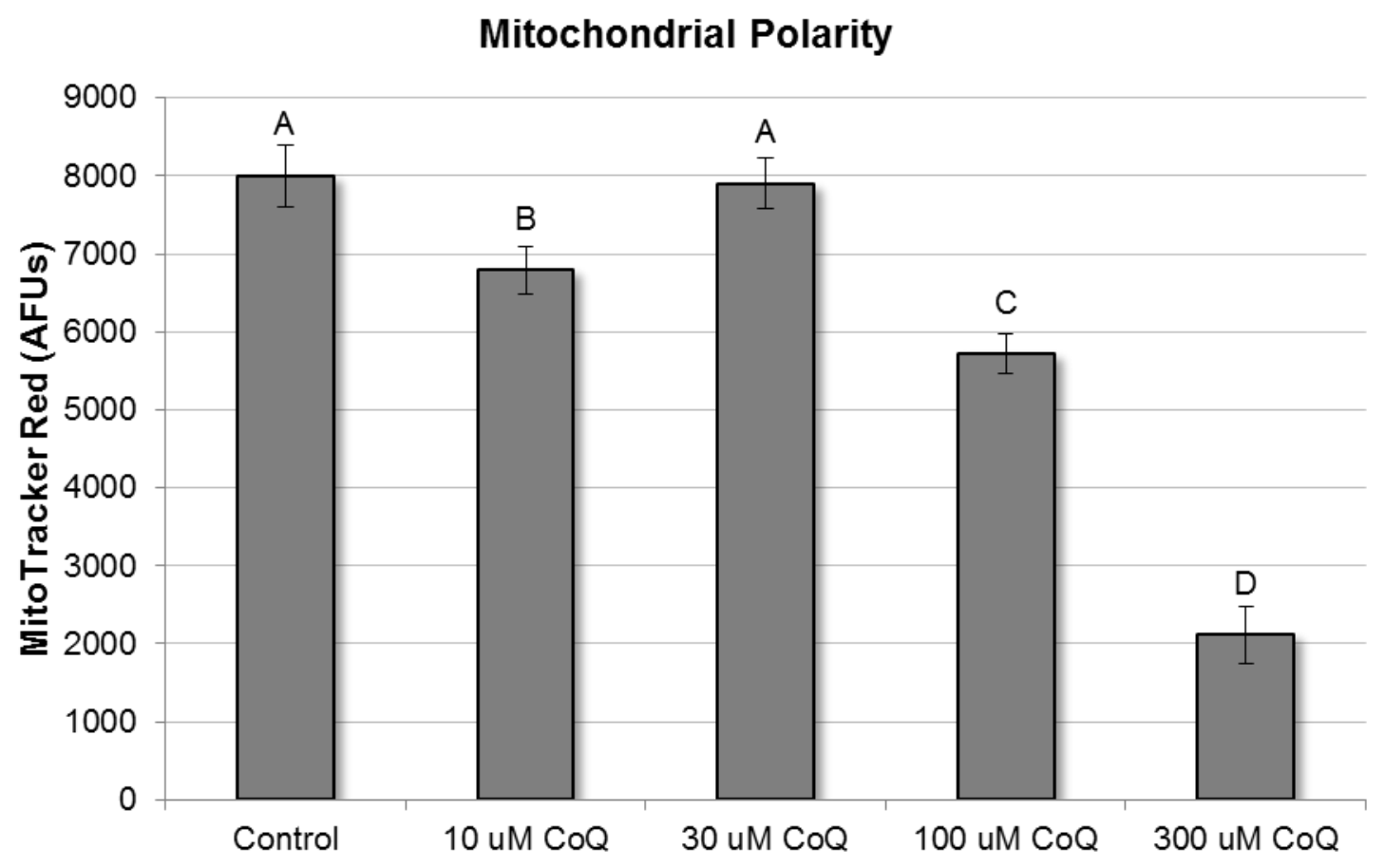

Figure 7. Mitochondrial polarity between CoQ concentrations. Means of mitochondrial polarity between treatment groups as represented by MitoTracker Red AFUs (Control $(n=57), 10 \mu M \operatorname{CoQ}(n=99), 30 \mu M \operatorname{CoQ}(n=89), 100 \mu M \operatorname{CoQ}(n=116)$, and $300 \mu M \operatorname{CoQ}$ $(\mathrm{n}=68))$. Error bars represent standard error of the mean, and values without common labels differ $(\mathrm{P}<0.05)$. 


\subsection{Discussion}

In this study, both $10 \mu \mathrm{M}$ and $30 \mu \mathrm{M}$ CoQ decreased lipid content of embryos. The overall benefit of this media additive was found to be limited, however, as there was no concurrent increase in blastocyst percentage or mitochondrial polarity with these treatments, which are believed to be markers for an increase in embryo viability. Previous studies have demonstrated that $\mathrm{CoQ}$ increases the proportion of oocytes that reach the blastocyst stage in a concentration-dependent manner, with $10 \mu \mathrm{M}$ and $30 \mu \mathrm{M}$ CoQ being the most effective concentrations, and $100 \mu \mathrm{M} \mathrm{CoQ}$ showing a decrease in blastocyst percentage (Stojkovic et al., 1999). It is believed that CoQ contributes to cell proliferation and growth by promoting ATP production within the inner mitochondrial membrane and balancing the redox state of cells (Schneider, Lemasters, \& Hackenbrock, 1982; Sun, Sun, \& Crane, 1992).

The present study did not validate the proliferative effects seen by other researchers. Coenzyme $Q_{10}$ is extremely lipophilic and resides in the intermembrane space of the inner mitochondrial membrane, making the delivery of this substance to its location of action a challenge. A previous study prepared a submicron-sized dispersion of CoQ for culture with bovine embryos to increase its bioavailability (Stojkovic et al., 1999). Other studies examining the effects of CoQ on cell lines have used liposomal CoQ as a delivery method (Schneider et al., 1982), which was the method used in the present study. Because liposomes pass through cellular membranes and deliver CoQ by fusing with the existing phospholipid bilayer, the length of the inner mitochondrial membrane increases as a result. This increase in length may also increase the distance between integral proteins involved in electron transport, thereby slowing the rate of metabolism occurring in the cell, which may explain the decrease in mitochondrial polarity seen as the concentration of liposomal CoQ increased (Schneider et al., 1982). Overall, the delivery of $\mathrm{CoQ}$ by liposomes was not shown to be an operative method of producing the positive 
effects of $\mathrm{CoQ}$ on embryo development, although a concentration of $30 \mu \mathrm{M}$ was the most effective relative to other concentrations examined. As a result of this pilot study, it was concluded that other metabolic regulators should be examined for their effectiveness in decreasing lipid content and increasing mitochondrial polarity, both individually and combined in comparison to CoQ. Additional factors affecting the evaluation of effects seen from culture with or without metabolic regulators should also be examined, such as stage of development at the time of evaluation and media type used for culture. 


\section{Chapter 4. Pilot Study 2: Media Type, Additive, Stage}

\subsection{Introduction}

The conditions of post-fertilization embryo culture are the most significant factors affecting blastocyst quality (Rizos, 2002). Sequential culture media was developed to accommodate the embryo's preference for oxidative phosphorylation during the 1-8 cell stage by providing pyruvate as an energy source (J. G. Thompson, 2000). Embryos are then transferred to a medium which provides a higher concentration of glucose so that glycolysis can occur during compaction and blastulation (J. G. Thompson, 2000). The culture of embryos in either sequential or continuous media may have an effect on the overall yield of blastocysts per oocyte, and may also affect the action of metabolic regulators added to the media.

At the beginning of the blastocyst stage, activation of $\mathrm{Na}+\mathrm{K}+$ pumps is necessary for the import of water to form the blastocoel cavity. Additionally, macromolecule synthesis increases as cell lineages are formed (Houghton \& Leese, 2004). These energydemanding processes coincide with a switch to glucose as a substrate for ATP production. Lipid content decreases at this stage as free fatty acids are used as an energy source (Jeong et al., 2009). Lipid content then continues to decrease as the embryo expands (Jeong et al., 2009). Because of this stage-dependent change in triglyceride accumulation, it is important to know if alterations to the culture conditions show the same effects on both early (stage 6) and late (stage 7) stage blastocysts.

In vitro-produced Holstein embryos accumulate an excess of intracellular lipids, which negatively impacts their ability to withstand cryopreservation (Abe et al., 2002; Barceló-Fimbres \& Seidel, 2007a, 2007b; Enright et al., 2000; E M Ferguson \& Leese, 1999; Jeong et al., 2009). Chemical delipation through the addition of L-carnitine or forskolin is an approach for improving the quality and cryotolerance of bovine IVP embryos (Sanches et al., 2013; T. Takahashi et al., 2013). L-carnitine facilitates the transfer of free 
fatty acids into the mitochondria for subsequent $\beta$-oxidation and metabolism (SuttonMcDowall et al., 2012). Forskolin promotes lipolysis through an increase in intracellular cAMP, which activates key lipase enzymes that break triglycerides into free fatty acids (Dunning et al., 2014; Men et al., 2006).

ATP production regulates the embryo's progression through its first cellular divisions, activation of its native genome, and compaction of the morula, which involves establishing cell-to-cell contacts and differentiation of cell types into the trophectoderm and inner cell mass (Baldoceda-Baldeon et al., 2014; Dumollard et al., 2009; Gardner et al., 2000; Rieger, 1992; Rizos, 2002; Sutton-McDowall et al., 2012; Van Blerkom, 2004). Mitochondrial dysfunction may contribute to an accumulation of intracellular lipids as a result of lowered mitochondrial activity (Luis Baldoceda et al., 2016). Vitamin $\mathrm{K}_{2}$ is believed to work through a similar mechanism as coenzyme $Q_{10}$, restoring proper function of the electron transport chain by serving as an electron carrier (Vos et al., 2012). By repairing dysfunctional mitochondria, CoQ and/or VitK may allow for mitochondria to continue the metabolism of free fatty acids when they would have otherwise halted, decreasing the accumulation of triglycerides within the cell.

Chemically defined medium provides a way of studying the individual effects of specific growth factors or metabolic regulators on embryo development and ATP production (Lim et al., 2007). The objectives of this study were: 1) to determine if culturing embryos in a continuous versus sequential media has an effect on blastocyst yield or lipid accumulation, 2) to determine if the stage of development at which embryos are assessed affects the results of the study, and 3) to compare the effects of various metabolic regulators as additives to embryo culture. This study was separated into two parts to address these objectives. It was hypothesized that sequential media would increase blastocyst yield and decrease lipid accumulation, that stage of development at 
assessment would not affect results observed, and that each additive would cause a decrease in lipid content and an increase mitochondrial polarity. 


\subsection{Materials and methods}

\subsubsection{In vitro embryo production}

Embryos were produced from oocytes $(n=5070$, part $1 ; n=3100$, part 2 ) as described in Chapter 2.2 with the following modifications.

\subsubsection{Addition of L-carnitine}

A $50 \mathrm{mM}$ stock of L-carnitine (C0283; Sigma) was prepared and stored at $-80^{\circ} \mathrm{C}$ until the day of use. After fertilization, presumptive zygotes were randomly assigned to culture medium that contained either $0 \mathrm{mM} \mathrm{L}$-carnitine or $0.5 \mathrm{mM} \mathrm{L}$-carnitine and cultured for 7 to 9 days.

\subsubsection{Addition of coenzyme $Q_{10}$}

A $0.115 \mathrm{M}$ stock of $\mathrm{CoQ}$ (Homogenous liposomal ubiquinol, Tishcon Corp, Westbury, NY, USA) was diluted to a $1.5 \mathrm{mM}$ intermediate solution using embryo culture medium. At day 3 after fertilization, CoQ was added to randomly assigned culture drops containing 20-25 fertilized oocytes at a final concentration of $30 \mu \mathrm{M}$.

\subsubsection{Addition of vitamin $K_{2}$}

A 200 mM stock was prepared by dissolving vitamin $K_{2}$ powder (V9378; Sigma) into $95.5 \%$ ethanol and freezing at $-80^{\circ} \mathrm{C}$. Vitamin $\mathrm{K}_{2}$ was added to randomly assigned embryo culture groups at day 3 after fertilization at a final concentration of $0.5 \mathrm{mM}$.

\subsubsection{Addition of forskolin}

A 10 mM stock of forskolin was prepared by dissolving forskolin (344270; Sigma) into dimethyl sulfoxide solution and storing at $-80^{\circ} \mathrm{C}$. Forskolin was added to randomly assigned embryo culture groups at a final concentration of $10 \mu \mathrm{M}$ on day 5 after fertilization.

\subsubsection{Evaluation of embryos}

Lipid content and mitochondrial polarity of embryos was evaluated as described in Chapter 2.3.2-2.3.3 with the following specifications. 


\subsubsection{Lipid content}

Lipid accumulation was quantified by staining blastocysts with the lipophilic Nile Red dye as described in Chapter 2.3.2. For part 1, images were acquired along the equatorial plane of the embryo at $40 \mathrm{X}$ magnification using a widefield fluorescent microscope equipped with a FITC filter (Zeiss Axioskop 40, Oberkochen, Germany). For part 2, images were acquired by confocal microscopy at $40 \mathrm{X}$ magnification using an excitation/emission wavelength of $488 / 520$ (Olympus Fluoview, Tokyo, Japan). Ten orthogonal projections along the equatorial section of the embryo were collected with a 5 $\mu \mathrm{m}$ step size.

For both parts, fluorescence was measured using ImageJ software (National Institutes of Health, Bethesda, MD, USA) with background fluorescence corrected for, and mean fluorescence between treatment groups was analyzed. Results are expressed in mean fluorescence arbitrary units (AFUs).

\subsubsection{Mitochondrial polarity}

Mitochondrial polarity was assessed using MitoTracker Red CMX-rosamine dye, which binds with varying affinity based on degree of mitochondrial polarity, as described in Chapter 2.3.3. Images were acquired by confocal microscopy at $40 \mathrm{X}$ magnification using an excitation/emission wavelength of 594/608 (Olympus Fluoview, Tokyo, Japan). Ten orthogonal projections along the equatorial section of the embryo were collected with a $5 \mu \mathrm{m}$ step size. Fluorescence intensity was measured using ImageJ software (National Institutes of Health, Bethesda, MD, USA) with background fluorescence corrected for, and mean fluorescence between treatment groups was analyzed. Results are expressed in mean fluorescence intensity arbitrary units (AFUs).

\subsubsection{Experimental design}

This experiment was designed in two parts. For part 1, a $2 \times 2 \times 2$ factorial design was used to compare culture systems (continuous vs. 3-step sequential), blastocyst stage 
(stage 6 (early) vs. stage 7 (late)), and cumulative additives (no additives vs. L-carnitine (0.5 $\mathrm{mM}$ at day 1$)$, plus coenzyme $\mathrm{Q}_{10}(30 \mu \mathrm{M}$ at day 3$)$, plus vitamin $\mathrm{K}_{2}(0.5 \mathrm{mM}$ at day 3), plus forskolin (10 $\mu \mathrm{M}$ at day 5)). Total lipid content was used to evaluate embryos from each treatment group.

For part 2, a $2 \times 2 \times 2 \times 2$ factorial design was used to compare each additive (none vs. $0.5 \mathrm{mM}$ L-carnitine at day 1 , none vs. $30 \mu \mathrm{M} \mathrm{CoQ}$ at day 3 , none vs. $0.5 \mathrm{mM}$ VitK at day 3, and none vs. $10 \mu \mathrm{M}$ forskolin at day 5) as well as all combinations of these additives. Mitochondrial polarity was used to evaluate late stage (stage 7) blastocysts from each treatment group.

\subsubsection{Statistical analysis}

Lipid content and mitochondrial polarity were evaluated by one way-ANOVA using the SAS statistical software package general linear model (GLM; SAS Institute, Cary, NC, USA). If the ANOVA was significant $(P<0.05)$, means were separated by Tukey's HSD. 


\subsection{Results}

\subsubsection{Media type (Part 1)}

No effect $(P>0.05)$ was seen between lipid content in embryos cultured in continuous or 3-step sequential media (33.78 \pm 0.90 AFUs vs. $32.40 \pm 1.00$ AFUs, respectively) [Table 1]. This result held true when examining the interaction between stage and media type, as well as additives and media type [Table 1]. After it was determined that media type did not have an effect on lipid accumulation, this factor was removed from inclusion in further results analyses and a continuous media was then used for part 2 of this study.

\subsubsection{Stage of development (Part 1)}

Late stage embryos had less $(\mathrm{P}<0.05)$ intracellular lipid accumulation than did early stage embryos in both the additive and the no additive groups. Early stage embryos had a mean lipid content of $43.81 \pm 1.62$ AFUs when cultured with no additives, and a mean of 33.41 \pm 0.80 AFUs when cultured with additives [Fig. 8]. Late stage embryos had a mean lipid content of $31.33 \pm 1.80$ AFUs when cultured with no additives and a mean of 23.79 \pm 0.85 when cultured with additives [Fig. 8].

\subsubsection{Media additives (Parts 1 and 2)}

For part 1, a combination of L-carnitine, CoQ, VitK, and forskolin decreased mean lipid content in both early (33.41 \pm 0.80 AFUs vs. $43.81 \pm 1.62$ AFUs (no additive)) and late stage $(23.79 \pm 0.85$ vs. $31.33 \pm 1.80$ AFUs (no additive)) embryos [Fig. 8\&10]. Culturing embryos in the presence of additives reduced the lipid content of early stage embryos to be similar to the lipid content of late stage embryos cultured with no additives present [Fig. 8]. For part 2, coenzyme $Q_{10}$, vitamin $K_{2}$, and forskolin all increased $(P<0.05)$ mitochondrial

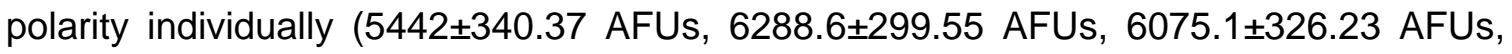
respectively vs. $3351.6 \pm 328.12$ AFUs (no additive)), however, these additives showed no 
effect $(P>0.05)$ when combined (3552.5 \pm 227.4 AFUs (all additives) vs. 3351.6 \pm 328.12 AFUs (no additive)) [Fig. 9\&10]. 


\begin{tabular}{|c|c|c|c|c|c|}
\hline & Interaction & Media Type & $\mathbf{N}$ & Nile Red AFUs & SE \\
\hline \multirow{4}{*}{ a) } & None & Continuous & 149 & 33.78 & \pm 0.90 \\
\hline & None & 3-Step Sequential & 189 & 32.40 & \pm 1.00 \\
\hline & Early (6) & Continuous & 80 & 39.34 & \pm 1.17 \\
\hline & Early (6) & 3-Step Sequential & 101 & 37.88 & \pm 1.27 \\
\hline \multirow[t]{4}{*}{ b) } & Late (7) & Continuous & 69 & 28.21 & \pm 1.30 \\
\hline & Late $(7)$ & 3-Step Sequential & 88 & 26.91 & \pm 1.35 \\
\hline & Additive & Continuous & 112 & 29.38 & \pm 0.90 \\
\hline & Additive & 3-Step Sequential & 163 & 27.82 & \pm 0.74 \\
\hline \multirow[t]{2}{*}{ c) } & No Additive & Continuous & 37 & 38.18 & \pm 1.56 \\
\hline & No Additive & 3-Step Sequential & 26 & 36.97 & \pm 1.86 \\
\hline
\end{tabular}

Table 1. Lipid content between groups. (Part 1) No difference $(P>0.05)$ was seen between the mean lipid content of all embryos cultured in continuous media versus the mean lipid content of all embryos cultured in 3-step sequential media (a). No difference $(P>0.05)$ was seen between early stage embryos or late stage embryos cultured in continuous versus 3 -step sequential media (b). No difference $(P>0.05)$ was seen between embryos cultured with additives or with no additives in continuous versus 3-step sequential media (c). 


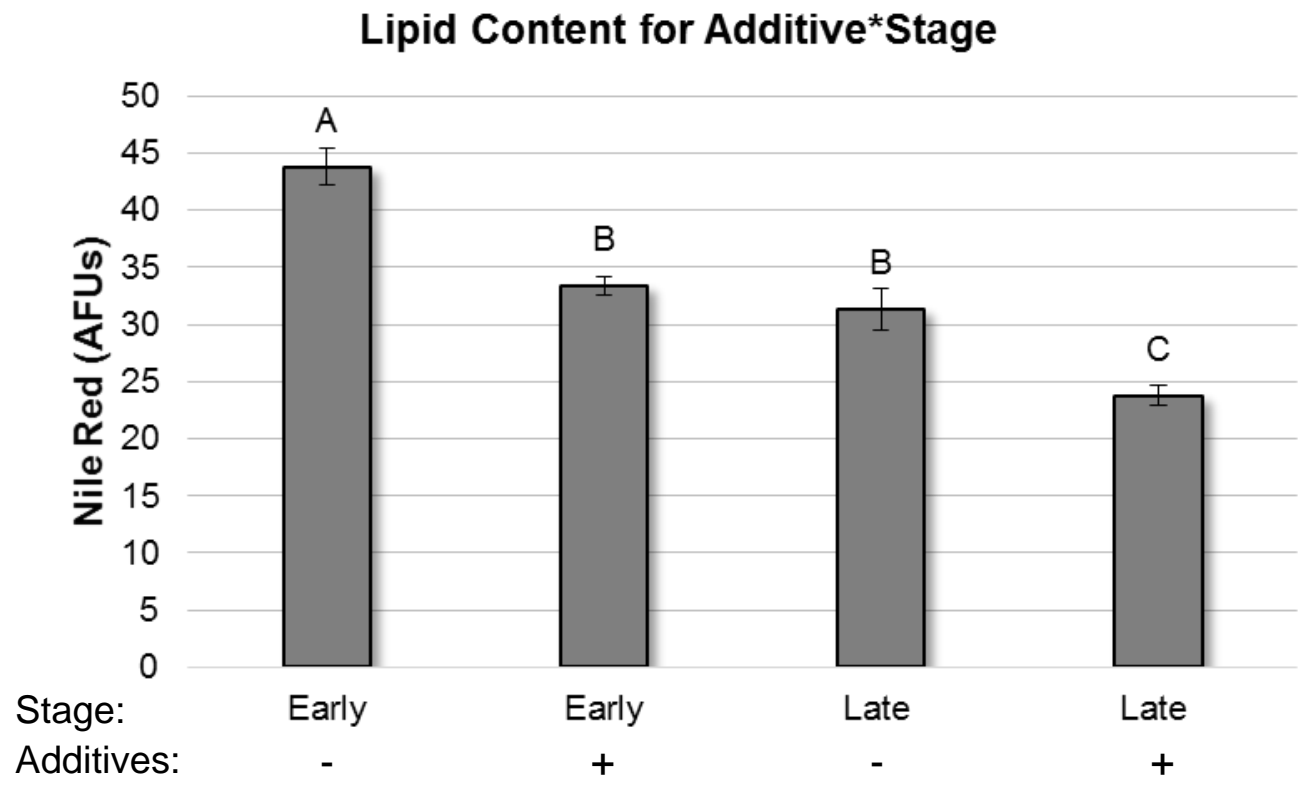

Figure 8. Lipid content between groups. (Part 1) Lipid content, as expressed by mean arbitrary fluorescent units (AFUs) of Nile Red in early stage embryos cultured with no additives $(n=35)$, early stage embryos cultured with additives $(n=146)$, late stage embryos with no additives $(n=28)$, and late stage embryos with additives $(n=129)$. Error bars represent standard error of the mean, and values without common labels differ $(P<0.05)$. 


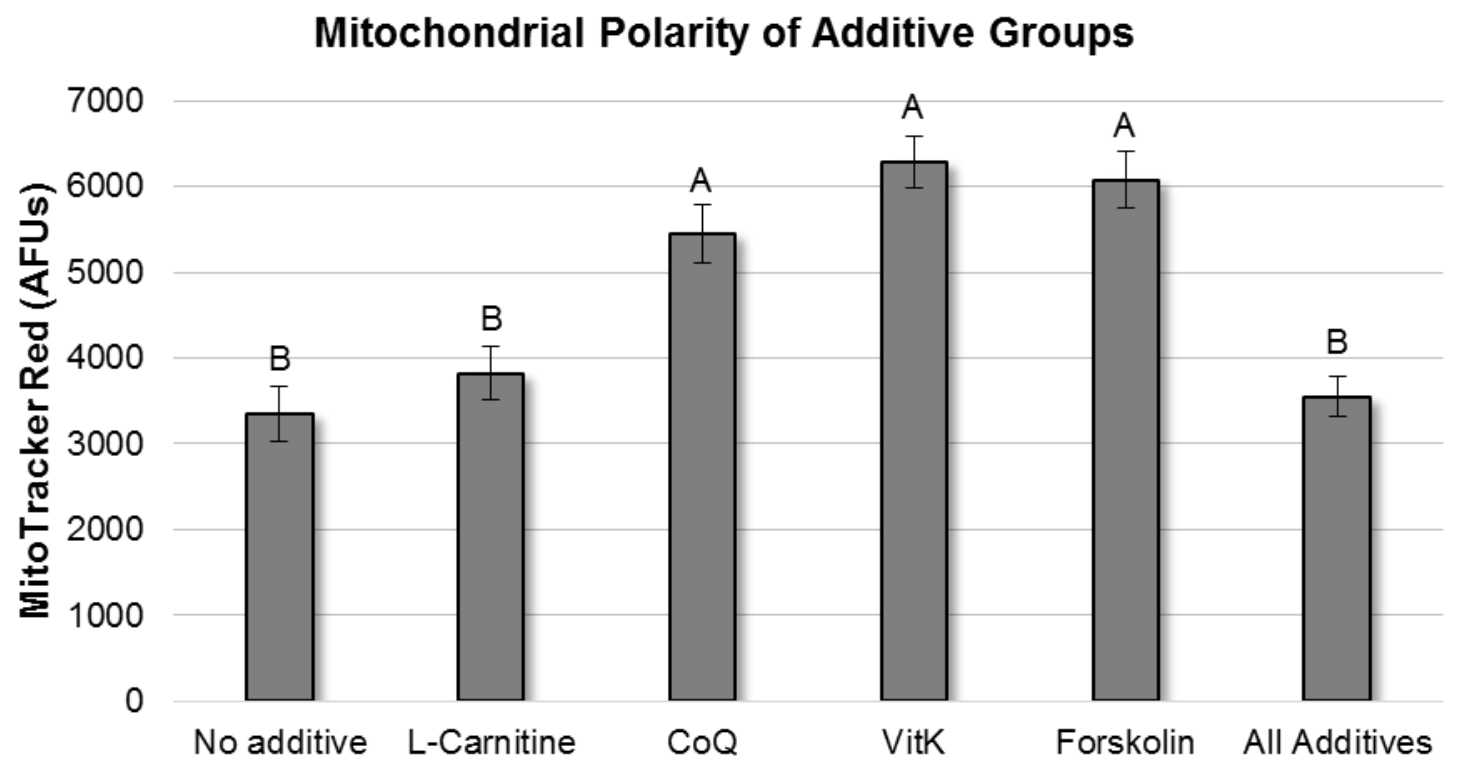

Figure 9. Mitochondrial polarity between groups. (Part 2) Mean mitochondrial polarity of embryos cultured in the presence of no additive $(n=85)$, L-carnitine $(n=91)$, coenzyme $Q_{10}(n=79)$, vitamin $K_{2}(102)$, forskolin (86), and a combination of all additives (177), as represented by arbitrary fluorescent units (AFUs) of MitoTracker Red. Error bars represent standard error of the mean, and values without common labels differ $(\mathrm{P}<0.05)$. 


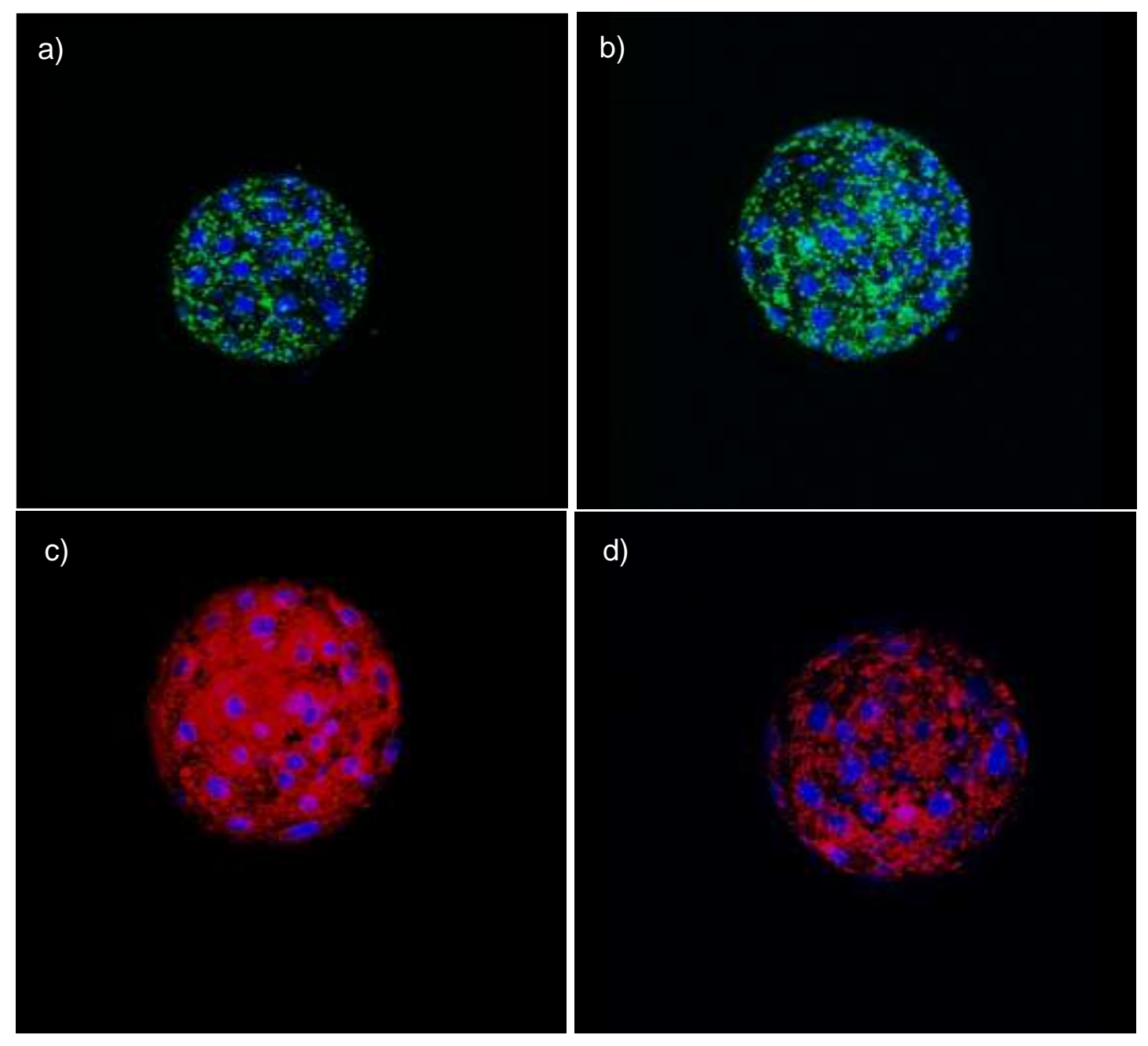

Figure 10. Representative images. Images showing intracellular lipid (Nile Red, green), nuclei (DAPI, blue), and mitochondrial polarity (MitoTracker Red, red). Stage 7 embryo cultured in the presence of additives (a) showing decreased intracellular lipids in comparison to a stage 7 embryo cultured with no additives (b). Stage 7 embryo cultured in the presence of vitamin $\mathrm{K}_{2}$ (c) showing increase mitochondrial polarity in comparison to a stage 7 embryo cultured with no additives (d). 


\subsection{Discussion}

The changing nature of embryo physiology and nutrient requirements during preimplantation development certainly justify the concept of sequential media to provide the specific nutrients required at various stages (Gardner et al., 2000). There are several mechanisms that control nutrient uptake in the embryo, which include the concentration and availability of these nutrients in the embryo's environment, as well as the regulation of enzymatic activity in response to this environment (Gardner et al., 2000). The latter is the most important way in which an embryo adapts to new surroundings (Gardner et al., 2000). Altering the nutrient provision to the embryo throughout development may help to alleviate stress on the embryo through these adaptation mechanisms (Gardner et al., 2000; Sutton-McDowall et al., 2012). However, while this sequential media system may provide the proper timing and ratio of exogenous energy sources, it does not facilitate the embryo's metabolism of endogenous lipids (Sutton-McDowall et al., 2012).

There was a numerical but insignificant decrease in lipid accumulation seen in each category of embryos (early vs. late, additives vs. no additives) that were cultured in 3-step sequential media as opposed to continuous media [Table 1]. This supports findings which indicate that improving nutrient supply may reduce accumulation of lipid droplets in bovine embryos (Abe et al., 2002). To see a significant change in lipid accumulation, however, the metabolism of these triglycerides must be facilitated. The groups of embryos cultured in the presence of additives exhibited a significant decrease in lipid accumulation at both the early and late stage of development. Forskolin activates adenylate cyclase on the membranes of cells, which increases intracellular cAMP and creates a signal cascade that ends in the activation of key lipase enzymes which control the breakdown of triglycerides into free fatty acids (Men et al., 2006). These free fatty acids require Lcarnitine for translocation into the mitochondria by carnitine palmitoyl transferase $1 \mathrm{~B}$ (Sutton-McDowall et al., 2012). Providing L-carnitine as a $\beta$-oxidation cofactor in addition 
to forskolin to generate free fatty acids for metabolism are likely the most significant effectors of the decrease in lipid accumulation seen in this portion of the experiment.

Embryos utilize endogenous lipids as a source of energy throughout development, so it would follow that lipid accumulation decreases over time as these stores deplete, consistent with the findings in part 1 of this experiment examining the effect of stage on lipid accumulation (Abe et al., 2002; Jeong et al., 2009). However, there seems to be a disproportionately low utilization of these endogenous lipids in IVP embryos as compared to in vivo-produced embryos (Gad et al., 2012). While improper mitochondrial function may be a factor in this disproportionate lipid consumption, the morphology and functional efficiency of mitochondria at various stages of embryo development are only vaguely defined (B. D. Bavister \& Squirrell, 2000). In this pilot study, it was found that a combination of all additives decreased lipid content in both early and late stage blastocysts.

Culturing embryos in the presence of $\mathrm{CoQ}$ and VitK alone resulted in an increase in mitochondrial polarity. This result was hypothesized, as both molecules work to transport electrons within the electron transport chain and facilitate the pumping of hydrogen ions across the inner mitochondrial membrane, generating a polar gradient (Baldoceda-Baldeon et al., 2014; Stojkovic et al., 1999; Vos et al., 2012). Forskolin was also found to increase mitochondrial polarity, which may occur by providing cells with free fatty acids from triglycerides that are available to undergo $\beta$-oxidation and subsequent metabolism (Men et al., 2006).

Interestingly, both L-carnitine alone and a combination of all additives resulted in no change in mitochondrial polarity. With mitochondrial polarity being the only endpoint evaluated in part two of this study, there cannot be conclusive information noted regarding the reason for this occurrence. In part one of this study, a combination of all additives decreased lipid accumulation, showing a discrepancy between two of the endpoint markers of that are being used to evaluate the quality of these embryos. 
As a result of this study, it is evident that looking at only lipid accumulation or mitochondrial polarity does not give an inclusive portrayal of the simultaneous mechanisms involved in embryo metabolism and lipid accumulation. The ultimate goal is to generate a culture system which produces cryotolerant embryos. To date, the markers for cryotolerance are lipid accumulation and ROS. Further studies should include development rate, lipid content, mitochondrial polarity, and cryopreservation survival to evaluate the effectiveness of each metabolic regulator when used alone or in combination with others, as well as improve the relevance of embryo quality markers used. 


\section{Chapter 5. Experiment 1: Metabolic Regulators and Cryopreservation}

\subsection{Introduction}

The particular manifestation of IVC artifacts lends insight into the causes and potential approaches for improvement in blastocyst quality. The most prominent of these artifacts are lipid accumulation, increased presence of ROS, and limited development rates (Abe et al., 2002; Absalón-Medina et al., 2014; Prastowo et al., 2016; M. Sudano et al., 2013; M. Takahashi et al., 2000). These effects are a result of a multitude of factors, however, a perturbed energy metabolism pathway, mitochondrial dysfunction, and the lack of a niche environment may be the forefront causes of the differences seen between in vitro- and in vivo-derived embryos (Abe et al., 2002; Chen et al., 1994; Gardner et al., 2000; Prastowo et al., 2016).

Cryopreservation is essential for the widespread use of IVP bovine embryos in the embryo transfer industry (Fair et al., 2001), and the success of this cryopreservation greatly depends on blastocyst quality (T. Takahashi et al., 2013). Intracellular lipid accumulation is highly correlated with cryotolerance, however, the mechanism by which lipids interfere with cryopreservation is still poorly understood (Abe et al., 2002; BarcelóFimbres \& Seidel, 2007a; Seidel, 2006). What is known is that the conditions of in vitro culture seem to be the cause of this triglyceride accumulation, necessitating the optimization of IVC systems to improve blastocyst quality (Barceló-Fimbres \& Seidel, 2007a; Fair et al., 2001). Because of the correlation between blastocyst quality and cryotolerance, success rates of cryopreservation have been used and an indicator of embryo quality in studies of culture conditions (Rizos, 2002; Seidel, 2006)

A preliminary pilot study indicated that supplementation of embryo culture media with $30 \mu \mathrm{M}$ coenzyme $\mathrm{Q}_{10}$ decreases lipid content [Fig. 6] but does not have an effect on mitochondrial polarity [Fig. 7]. However, a subsequent pilot study showed that both CoQ and vitamin $\mathrm{K}_{2}$ can increase mitochondrial polarity when used individually [Fig. 9]. 
Because of the potential lengthening of the electron transport chain that can occur as a side effect of the delivery of CoQ in liposomal form (Schneider et al., 1982), it was decided that Vitamin $\mathrm{K}_{2}$ is a more optimal treatment for mitochondrial dysfunction. Vitamin $\mathrm{K}_{2}$ is believed to serve as an alternate electron carrier in a similar fashion as CoQ (Vos et al., 2012). By increasing electron transport chain efficiency, Vitamin $K_{2}$ can rescue cells from mitochondrial dysfunction (Vos et al., 2012).

Preliminary studies have also indicated that culturing embryos in the presence of L-carnitine and forskolin can decrease lipid content when compared to a control group [Fig. 8]. L-carnitine facilitates the action of CPT1B, which is the rate-limiting enzyme responsible for the transport of intracellular fatty acids into the mitochondria for metabolism (Sutton-McDowall et al., 2012). Forskolin may be able to work in conjunction with L-carnitine by providing the bioavailable free fatty acids to be metabolized. Forskolin activates adenylate cyclase, which creates a signal cascade that ends in the activation of hormone sensitive lipase, an enzyme responsible for cleaving the stores of intracellular triglycerides (Men et al., 2006).

Limited development rates are another inefficiency of in vitro culture. It is widely acknowledged that supplementation of embryo culture media with serum increases development relative to serum-free conditions (Absalón-Medina et al., 2014). However, the use of serum in culture media is also associated with lipid accumulation (Abe et al., 2002; Barceló-Fimbres \& Seidel, 2007b; E M Ferguson \& Leese, 1999) and large offspring syndrome (Lazzari, 2002). Co-culturing embryos with a cellular monolayer is one approach to providing embryos with the beneficial growth factors found in serum, such as tissue growth factor $\beta$ and insulin-like growth factor, without excess lipid supplementation (Abe et al., 2002). The cells within this monolayer secrete proteins into the media in which they are cultured, meaning this media can be removed from the cells and used to condition embryo culture media without adding the cells themselves (Chen et al., 1994). These 
additional factors may promote continued growth by providing proteins, growth factors, and signaling factors that would normally be present in the oviduct and uterus but are not present in in vitro culture. Vero cells are a cell line derived from Green monkey kidneys and are tested for viruses and other contaminants, making them a safe source for epithelial culture (Maeda et al., 1996).

The goal of improving bovine embryo culture is to generate embryos that are more similar in quality to in vivo-derived embryos, and to subsequently increase cryotolerance. The aim of the present study was to test the outcome of embryo culture with or without Lcarnitine, conditioned medium, VitK, forskolin, and all combinations of these additives, using cryopreservation survival as the ultimate marker of embryo quality. Development to the blastocyst stage, lipid accumulation, mitochondrial polarity, and percent of apoptotic cells after re-expansion will also be evaluated to provide insight into the quality of embryos in each treatment group. 


\subsection{Materials and methods}

\subsubsection{In vitro embryo production}

Embryos were produced from oocytes $(n=1229$, part $1 ; n=2461$, part 2 ) as described in Chapter 2.2 with the following modifications.

\subsubsection{Addition of L-carnitine}

A $50 \mathrm{mM}$ stock of L-carnitine (C0283; Sigma) was prepared and stored at $-80^{\circ} \mathrm{C}$ until the day of use. After fertilization, presumptive zygotes were randomly assigned to culture medium that contained either $0 \mathrm{mM} \mathrm{L-carnitine} \mathrm{or} 0.5 \mathrm{mM} \mathrm{L-carnitine} \mathrm{and} \mathrm{cultured}$ for 7 to 9 days.

\subsubsection{Conditioned media}

Vero cells (African Green Monkey kidney cells, Cercopithecus aethiops) were seeded in a four-well dish (Nunc, Copenhagen, Denmark) at a concentration of $1 \times 10^{5} / \mathrm{mL}$ using Tissue Culture Medium 199 (Life Technologies, Inc., Grand Island, NY, USA) supplemented with $5 \%$ fetal bovine serum. Cells were incubated at $37^{\circ} \mathrm{C}$ in humidified air containing $5 \% \mathrm{CO}_{2}$ for $48 \mathrm{hrs}$. Conditioned media was aspirated from the wells, filtered, aliquoted, and frozen at $-80^{\circ} \mathrm{C}$ until use. Conditioned media was added to culture media at a $5 \%$ v:v ratio, and embryos were randomly assigned to be cultured either in the presence or absence of conditioned medium starting at day 1 after IVF until stage 7 of development.

\subsubsection{Addition of vitamin $K_{2}$}

A 200 mM stock was prepared by dissolving vitamin $\mathrm{K}_{2}$ powder (V9378; Sigma) into $95.5 \%$ ethanol and freezing at $-80^{\circ} \mathrm{C}$. Vitamin $\mathrm{K}_{2}$ was added to randomly assigned embryo culture groups at day 3 after fertilization at a final concentration of $0.5 \mathrm{mM}$.

\subsubsection{Addition of forskolin}

A 10 mM stock of forskolin was prepared by dissolving forskolin (344270; Sigma) into dimethyl sulfoxide solution and storing at $-80^{\circ} \mathrm{C}$. Forskolin was added to randomly 
assigned embryo culture groups at a final concentration of $10 \mu \mathrm{M}$ on day 5 after fertilization.

\subsubsection{Cryopreservation and thawing}

Embryos were cryopreserved by standard slow freezing methods intended for direct transfer. Stage 7 blastocysts were equilibrated in $1.5 \mathrm{M}$ ethylene glycol plus $0.5 \mathrm{M}$ sucrose in holding media at $38.5^{\circ} \mathrm{C}$ for 10 minutes, then loaded into a $0.25 \mathrm{~mL}$ straw. Straws were cooled to room temperature during loading, then cooled to $-6.5^{\circ} \mathrm{C}$, seeded, and cooled at a rate of $0.3^{\circ} \mathrm{C} / \mathrm{min}$ using a programmable freezer (Crysalys; IMV Technologies, Normandy, France). After completion of the freezing protocol, embryos were plunged into liquid nitrogen.

Straws were thawed in air for 10 seconds, in $32^{\circ} \mathrm{C}$ water for 30 seconds, then the embryos were expelled from the straw and passed through a series of wash media drops to remove all ethylene glycol and sucrose. After washing, embryos were placed into preequilibrated embryo culture medium and placed back into culture for 36 hours. Post-thaw survival of embryos was assessed at 12,24 , and 36 hours of culture and recorded as a percentage of total embryos frozen per treatment group.

\subsubsection{Evaluation of embryos}

Development rates, lipid content, and mitochondrial polarity were evaluated as described in Chapter 2.3 with specifications as follows. Percent of apoptotic cells present in embryos after freezing and thawing was also used as an evaluation parameter.

\subsubsection{Lipid content}

Lipid accumulation was quantified by staining stage 7 blastocysts with the lipophilic Nile Red dye as described in Chapter 2.3.2. Images were acquired by confocal microscopy at $40 \mathrm{X}$ magnification using an excitation/emission wavelength of 488/520 (Olympus Fluoview, Tokyo, Japan). Ten orthogonal projections along the equatorial section of the embryo were collected with a $5 \mu \mathrm{m}$ step size. Fluorescence was measured using ImageJ 
software (National Institutes of Health, Bethesda, MD, USA) with background fluorescence corrected for, and mean fluorescence between treatment groups was analyzed. Results are expressed in mean fluorescence arbitrary units (AFUs).

\subsubsection{Mitochondrial polarity}

Mitochondrial polarity was assessed using MitoTracker Red CMX-rosamine dye, which binds with varying affinity based on degree of mitochondrial polarity, as described in Chapter 2.3.3. Images were acquired by confocal microscopy at 40X magnification using an excitation/emission wavelength of 594/608 (Olympus Fluoview, Tokyo, Japan). Ten orthogonal projections along the equatorial section of the embryo were collected with a $5 \mu \mathrm{m}$ step size. Fluorescence intensity was measured using ImageJ software (National Institutes of Health, Bethesda, MD, USA) with background fluorescence corrected for, and mean fluorescence between treatment groups was analyzed. Results are expressed in mean fluorescence intensity arbitrary units (AFUs).

\subsubsection{TUNEL assay}

Evaluation of apoptosis in frozen-thawed blastocysts was assessed by TUNEL assay (In Situ Cell Death Detection Kit, TMR Red; Roche, Basel, Switzerland). After cryopreservation and re-expansion of stage 7 blastocysts, surviving embryos were fixed in $10 \%$ paraformaldehyde $(\mathrm{v}: \mathrm{v})$ for at least 24 hours. TUNEL labelling was performed by washing the embryos three times in PBS-PVP, then treating with 0.5\% (v:v) Triton X-100 for $30 \mathrm{~min}$ at room temperature. Embryos were washed in PBS-PVP then incubated in a $25 \mu \mathrm{L}$ drop of TUNEL reaction mixture (containing enzyme solution and label solution in a $1: 9$ (v:v) ratio) for $1 \mathrm{hr}$ at $38.5^{\circ} \mathrm{C}$ in humidified air, protected from light, according to the manufacturer's instructions. Embryos were washed again in PBS-PVP and stained with 1 $\mu \mathrm{g} / \mathrm{mL}$ DAPI for $15 \mathrm{~min}$ at room temperature. As a positive control, embryos were treated with $1 \mathrm{IU} / \mu \mathrm{L}$ DNase (Promega Co., Madison, WI, USA), then incubated in a $25 \mu \mathrm{L}$ drop of 
reaction mixture as normal. As a negative control, embryos were incubated in a $25 \mu \mathrm{L}$ drop containing only the marking solution.

Finally, embryos were washed in PBS-PVP and mounted in a 1:10 (v:v) dilution of ProLong Diamond Antifade Mountant (ThermoFisher Scientific, Waltham, MA, USA) in PBS for imaging. Images were acquired by confocal microscopy at an excitation/emission wavelength of $594 / 608$ for the TUNEL channel and $350 / 470$ for the DAPI channel. Ten orthogonal projections along the equatorial section of the embryo were collected with a 5 $\mu \mathrm{m}$ step size. TUNEL and DAPI channels were overlain using ImageJ software (National Institutes of Health, Bethesda, MD, USA) and cells with overlapping nucleus staining were counted as TUNEL positive. Total cell number was calculated using a cell counter plug-in through the ImageJ software, and percentage of apoptotic cells was calculated as the number of TUNEL positive cells divided by the number of total cells counted.

\subsubsection{Experimental design}

This experiment was separated into two parts. In part 1, a $2 \times 2$ factorial design was used to compare the effects of conditioned medium ( $0 \%$ vs. $5 \%$ at day 1 after IVF) and L-carnitine ( $0 \mathrm{mM}$ vs. $0.5 \mathrm{mM}$ at day 1 after IVF). Blastocyst rate, lipid content, mitochondrial polarity, cryopreservation survival rate, and percentage of apoptotic cells after re-expansion were used to evaluate embryos from each treatment group. The experiment was performed in 8 replicates with cryopreservation performed in 6 .

For part 2, a $2 \times 2 \times 2$ factorial design was used to compare the effects of conditioned medium ( $0 \%$ vs. $5 \%$ at day 1$)$, vitamin $\mathrm{K}_{2}(0 \mathrm{mM}$ vs. $0.5 \mathrm{mM}$ at day 3$)$, and forskolin $(0 \mu \mathrm{M}$ vs. $10 \mu \mathrm{M}$ at day 5$)$. L-carnitine was held as a constant factor in part 2, and was present at a concentration of $0.5 \mathrm{mM}$ at day 1 after IVF in all treatments. Blastocyst rate, lipid content, mitochondrial polarity, cryopreservation survival rate, and percentage of apoptotic cells after re-expansion were used to evaluate embryos from each 
treatment group. The experiment was performed in 8 replicates with cryopreservation performed in 4 .

\subsubsection{Statistical analysis}

Data for blastocyst rate, re-expansion rate after cryopreservation, and percentage of apoptotic cells after re-expansion were transformed by the arcsine method $(\arcsin (\mathrm{SQRT}(\mathrm{x} / 100))$ then evaluated by factorial ANOVA to determine if interactions exist between treatment groups. If no interaction was present, data was analyzed by one-way ANOVA. Means were separated by LSD if the ANOVA was significant $(P<0.05)$. Data for lipid content and mitochondrial polarity was also evaluated by factorial ANOVA first, then one-way ANOVA if no interactions were present. Means were separated by LSD if the ANOVA was significant $(P<0.05)$. Analysis was performed using the SAS statistical software package general linear model (GLM; SAS Institute, Cary, NC, USA). 


\subsection{Results}

\subsubsection{L-carnitine and conditioned medium (Part 1)}

No difference $(P>0.05)$ was seen in blastocyst rates between treatment groups [Fig. 11]. Lipid content was reduced $(P<0.05)$ in embryos cultured in the presence of Lcarnitine (266.43 \pm 14.44 AFUs, control vs. $214.78 \pm 13.85$ AFUs, L-carnitine) [Fig. 12] which coincided with an increase $(P<0.05)$ in cryopreservation survival $(42.02 \pm 9.46 \%$, control vs. $72.32 \pm 9.46 \%$, L-carnitine) [Fig. 14]. There was no difference $(P<0.05)$ in mitochondrial polarity between the control and L-carnitine treatment groups $(6039.4 \pm$ 279.89 AFUs vs. $5530 \pm 265.53$ AFUs, respectively), while embryos cultured in conditioned media had decreased $(P<0.05)$ mitochondrial polarity $(4826.4 \pm 265.53$ AFUs $)$ [Fig. 13]. Embryos cultured with L-carnitine plus conditioned medium showed an increase $(\mathrm{P}<0.05)$ in mitochondrial polarity $(7210.1 \pm 289$ AFUs $)$ [Fig. 13]. Finally, there was no difference $(P>0.05)$ observed in the percentage of apoptotic cells between re-expanded embryos in any treatment group [Fig. 15].

\subsubsection{Vitamin $K_{2}$, forskolin, and conditioned medium (Part 2)}

After holding L-carnitine as a constant factor, embryos cultured with conditioned medium, vitamin $K_{2}$, and forskolin combined showed an increase $(P>0.05)$ in blastocyst rate compared to the control (39.4 $\pm 2.38 \%$ vs. $31.45 \pm 2.83 \%$, respectively) [Fig. 16]. Lipid content was reduced $(\mathrm{P}<0.05)$ in embryos cultured with only VitK, only forskolin, VitK + forskolin, conditioned medium + forskolin, and CM + VitK + forskolin (173.43 \pm 10.61 AFUs, $149.49 \pm 9.72$ AFUs, $143.11 \pm 10.61$ AFUs, $185.29 \pm 9.97$ AFUs, and $162.33 \pm 9.43$ AFUs, respectively) compared to the control (214.78 \pm 9.86 AFUs) [Fig. 17]. Lipid content increased $(P<0.05)$ in embryos cultured with only conditioned medium $(260.93 \pm 10.28$ AFUs) and was no different $(P>0.05)$ in embryos cultured with $\mathrm{CM}+\mathrm{VitK}(225.83 \pm 9.97$ AFUs) compared to the control (214.78 \pm 9.86 AFUs) [Fig. 17]. Mitochondrial polarity increased $(P<0.05)$ in the CM treatment group $(7210.1 \pm 248.14$ AFUs $)$, decreased 
$(\mathrm{P}<0.05)$ in the VitK + forskolin $(3716.4 \pm 248.14$ AFUs), $C M+$ forskolin $(4087.1 \pm 235.41$ AFUs), and CM + VitK + forskolin (4810.1 \pm 263.19 AFUs) treatment groups, and showed no difference $(P>0.05)$ in the rest of the treatment groups compared to the control $(5530$ \pm 235.41 AFUs) [Fig. 18].

Cryopreservation survival increased $(P<0.05)$ in embryos cultured in the presence of VitK + forskolin $(97.5 \pm 9.18 \%)$ compared to the control $(78.79 \pm 9.18 \%)$ and decreased $(P<0.05)$ in embryos cultured in the presence of conditioned medium $(54.45 \pm 9.18 \%)$ [Fig. 19]. The percentage of apoptotic cells present in re-expanded embryos decreased in embryos cultured with VitK + forskolin $(10.42 \pm 2.55 \%)$ compared to the control $(20.20 \pm$ 2.73\%) [Fig. 20]. 


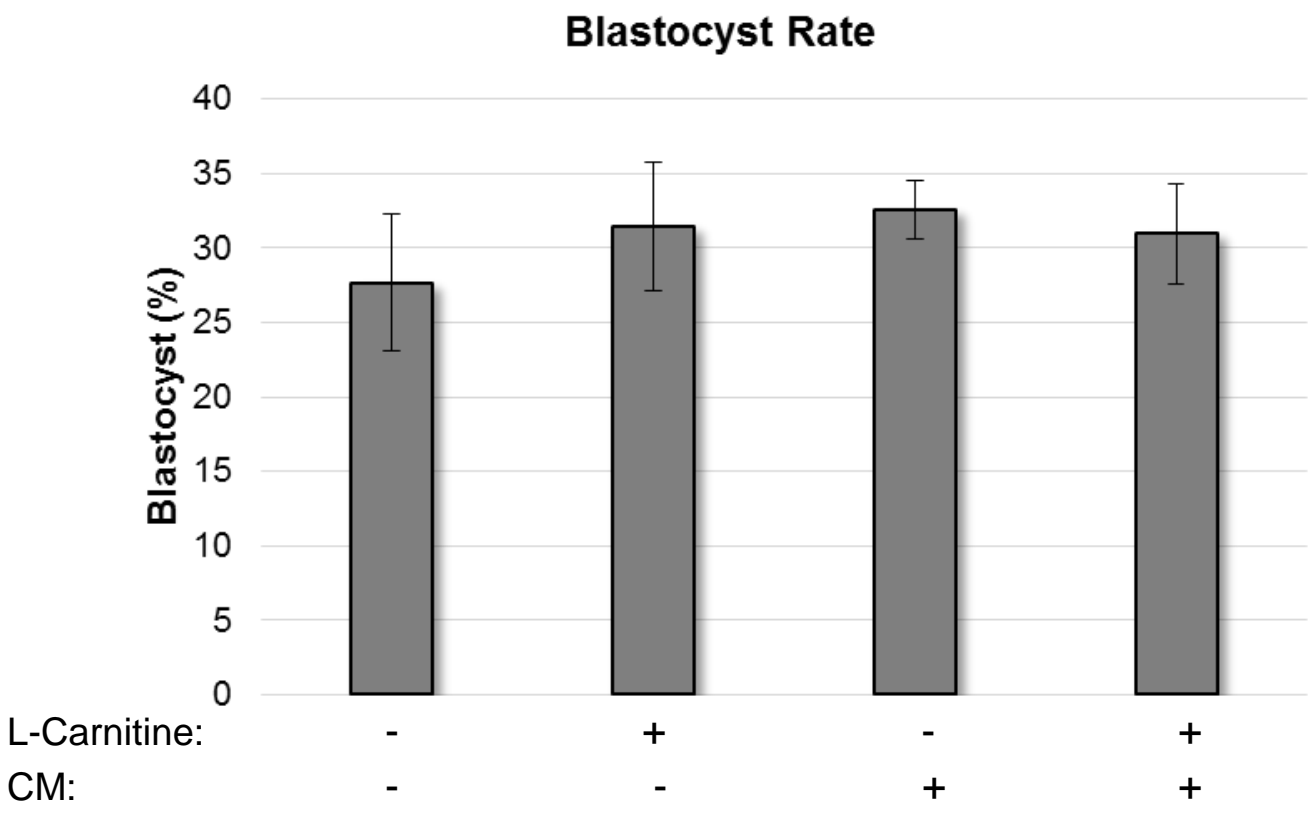

Figure 11. Blastocyst rate (Part 1). Blastocyst development rates between treatment groups did not differ $(P>0.05)$. Error bars represent standard error of the mean. $N=8$ replicates. $\mathrm{CM}=$ conditioned media. 


\section{Lipid Content}

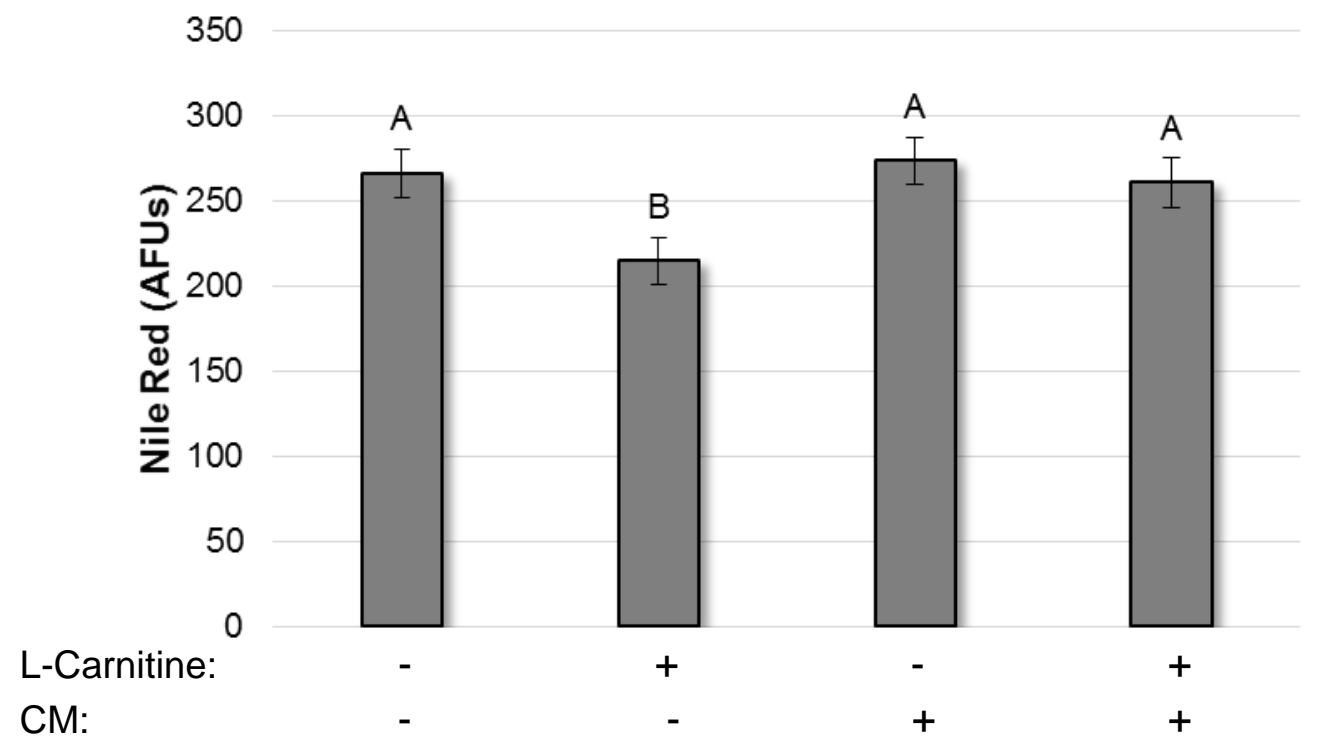

Figure 12. Lipid content (Part 1). Lipid content, as expressed by mean arbitrary fluorescent units (AFUs) of Nile Red, was decreased in embryos cultured in the presence of L-carnitine ( $n=174)$ compared to the control $(n=160)$, but did not change in the presence of conditioned medium (CM) $(n=180)$ or L-carnitine plus CM $(n=160)$. Error bars represent standard error of the mean, and values without common labels differ $(\mathrm{P}<0.05)$. 


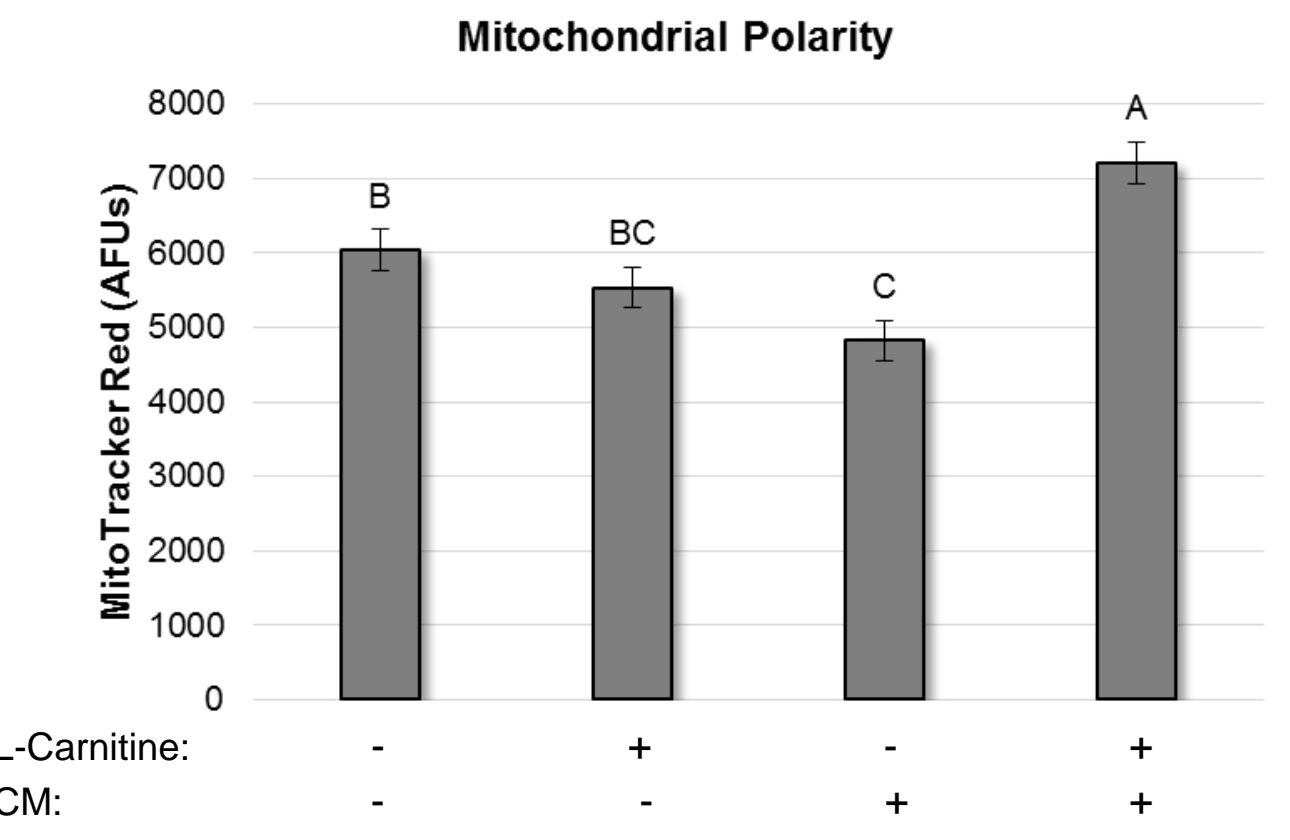

Figure 13. Mitochondrial polarity (Part 1). Mitochondrial polarity, as expressed by mean AFUs of MitoTracker Red was decreased in embryos cultured in conditioned medium (CM) ( $n=200)$ compared to the control $(n=180)$, and increased in embryos cultured in L-carnitine plus CM $(n=180)$. No difference was observed in embryos cultured with L-carnitine $(n=200)$. Error bars represent standard error of the mean, and values without common labels differ $(\mathrm{P}<0.05)$. 


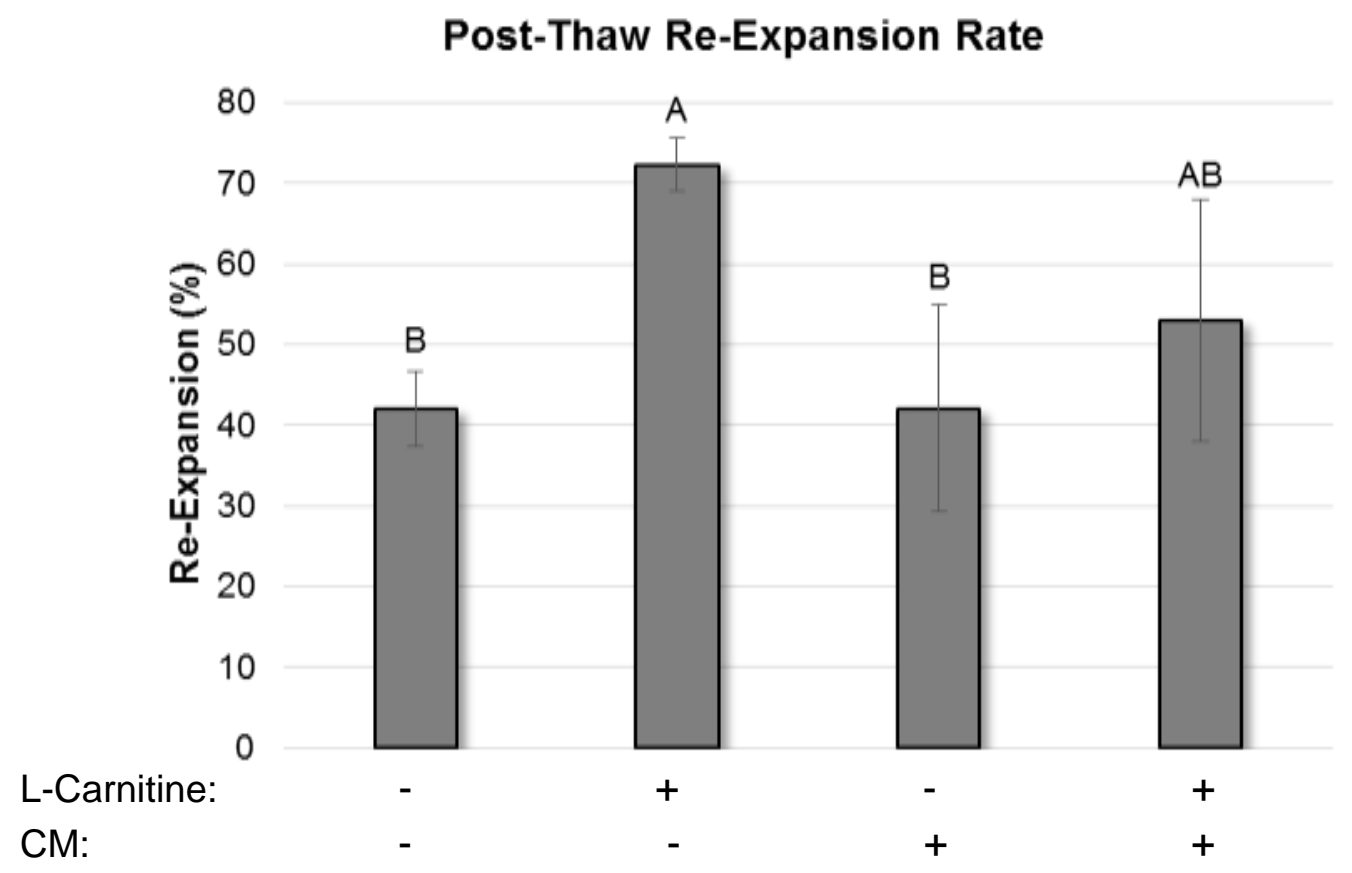

Figure 14. Re-expansion rate (Part 1). Post-thaw re-expansion rate was improved in embryos cultured in the presence of L-carnitine. $\mathrm{N}=6$ replicates. Error bars represent standard error of the mean, and values without common labels differ $(P<0.05)$. $C M=$ conditioned media. 


\section{Post-Re-Expansion Apoptotic Cells}

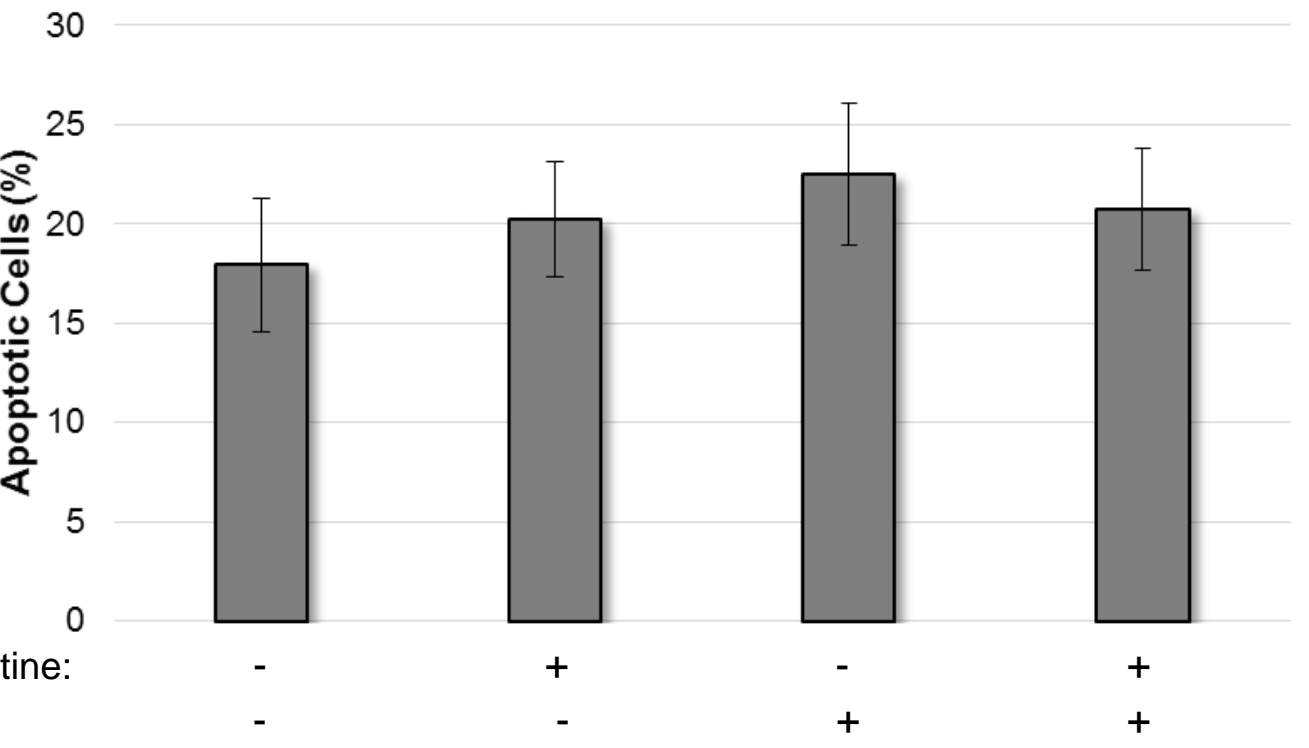

L-Carnitine:

CM:

Figure 15. Percent apoptotic cells (Part 1). No difference $(P>0.05)$ was seen in the percentage of apoptotic cells after re-expansion between the control $(n=25), L$-carnitine $(n=33), C M(n=22)$, and L-carnitine $+C M(n=30)$ treatment groups. Error bars represent standard error of the mean. $\mathrm{CM}=$ conditioned media. 


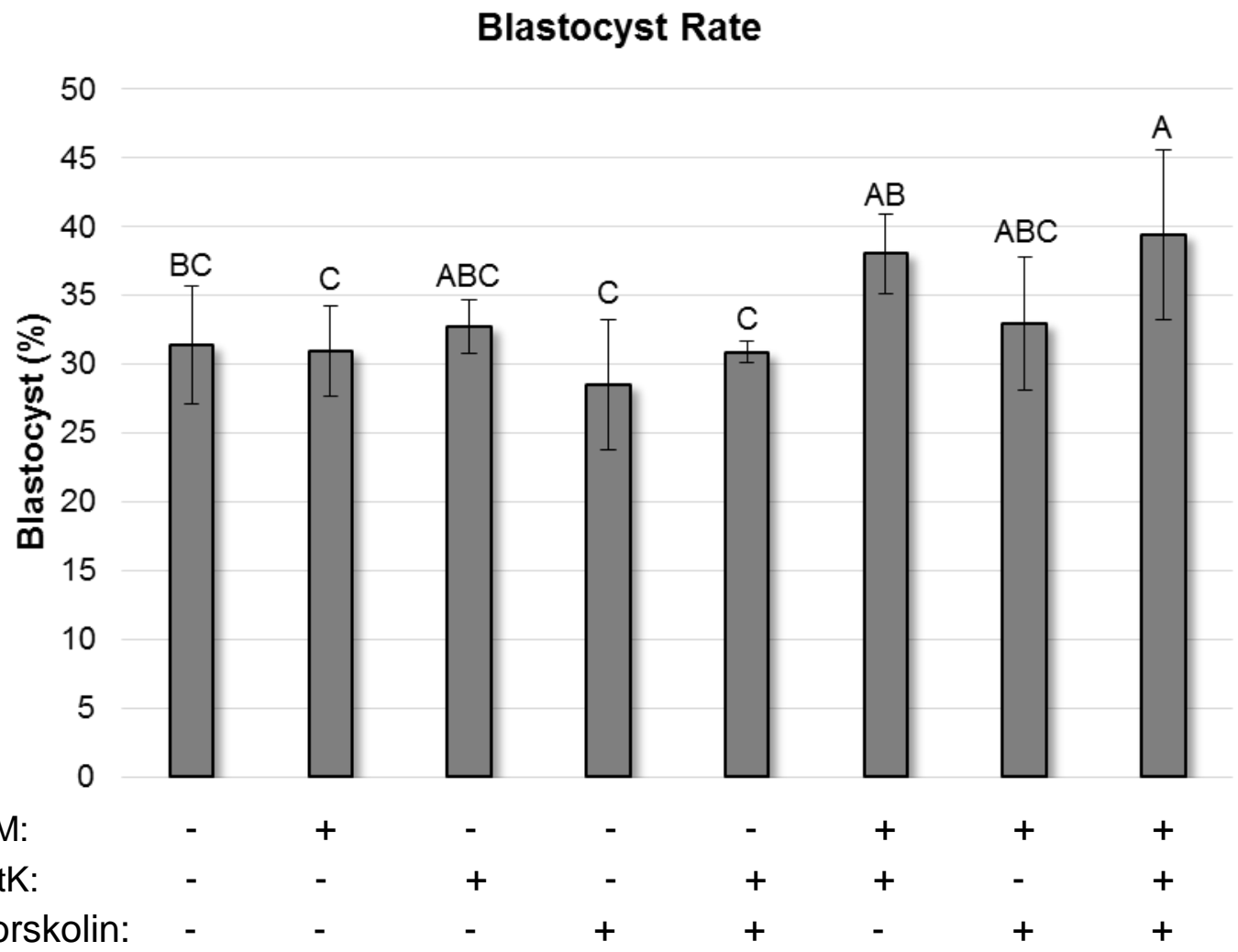

Figure 16. Blastocyst rate (Part 2). Blastocyst development rates between treatment groups. Values without common labels differ $(P>0.05)$. $N=8$ replicates. Error bars represent standard error of the mean. $\mathrm{CM}=$ conditioned media, VitK $=$ vitamin $\mathrm{K}_{2}$. 


\section{Lipid Content}

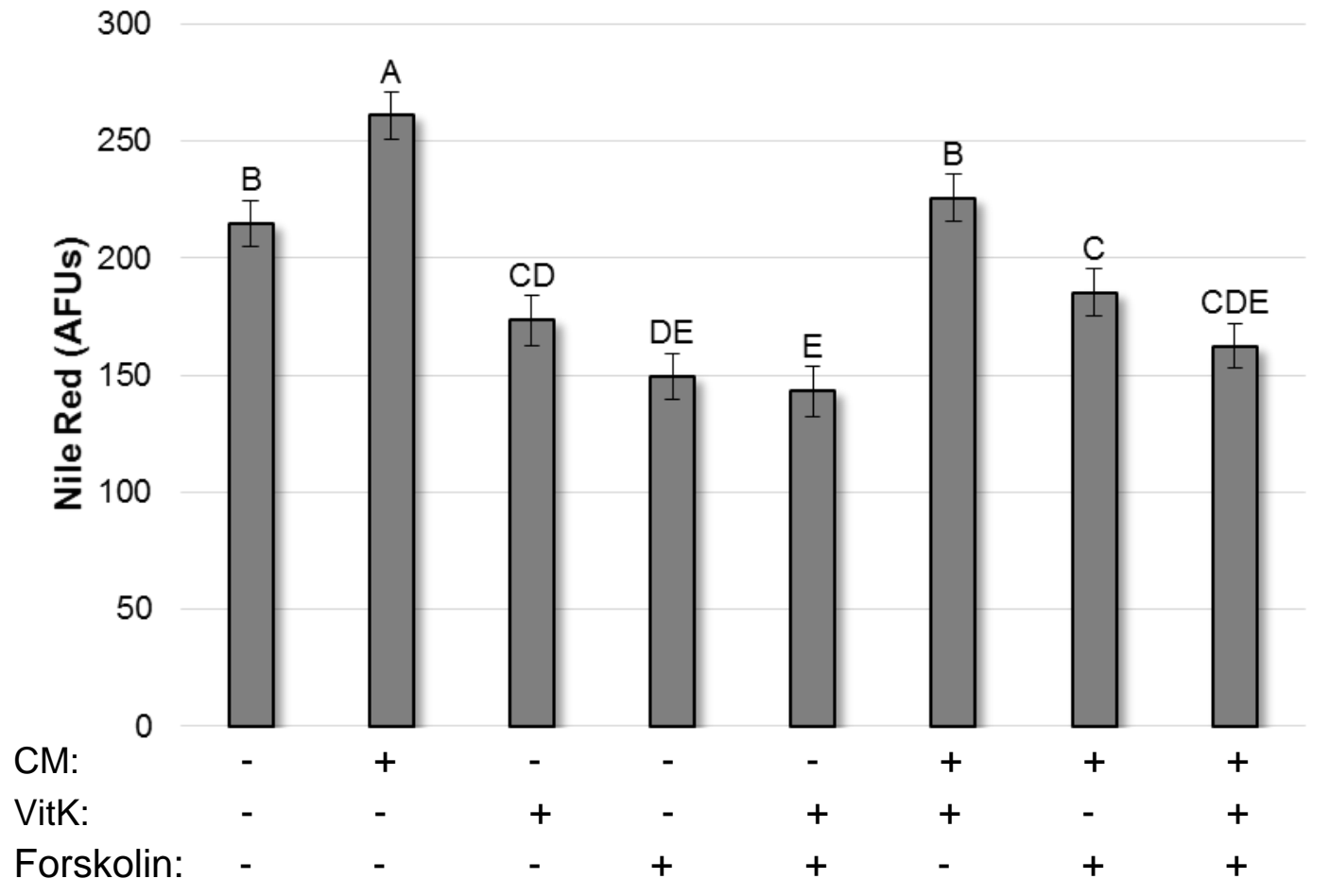

Figure 17. Lipid content (Part 2). Lipid content, as expressed by mean arbitrary fluorescent units (AFUs) of Nile Red, was decreased in vitamin $K_{2}$ (VitK) ( $\left.n=150\right)$, forskolin $(n=179)$, VitK + forskolin ( $n=150)$, conditioned medium (CM) + forskolin $(n=170)$, and CM + VitK + forskolin (190) treatment groups compared to the control $(n=174)$. No difference was observed in embryos cultured with CM + VitK $(n=170)$, and lipid content increased in the CM $(n=160)$ treatment group. Error bars represent standard error of the mean, and values without common labels differ $(\mathrm{P}<0.05)$. 


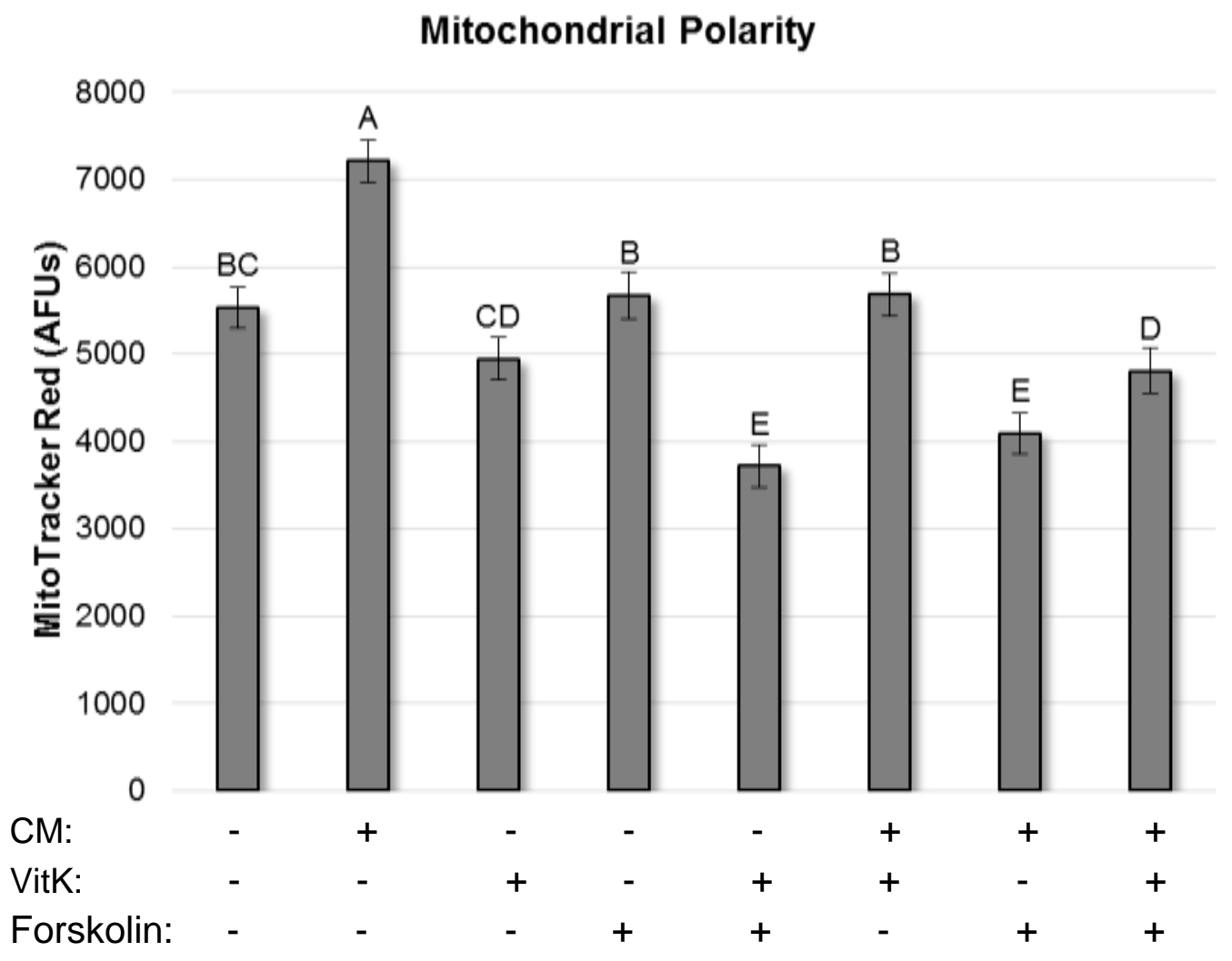

Figure 18. Mitochondrial polarity (Part 2). Mitochondrial polarity, as expressed by mean AFUs of MitoTracker Red was increased in the conditioned media (CM) $(n=180)$ treatment group and decreased in vitamin $K_{2}($ VitK $)+$ forskolin $(n=180), C M+$ forskolin $(n=200)$, and CM + VitK + forskolin $(n=160)$ treatment groups compared to the control $(n=200)$. No difference in mitochondrial polarity was observed in the VitK $(n=190)$, forskolin $(n=160)$, or CM + VitK $(n=190)$ treatment groups. Error bars represent standard error of the mean, and values without common labels differ $(P<0.05)$. 


\section{Post-Thaw Re-Expansion Rate}

120

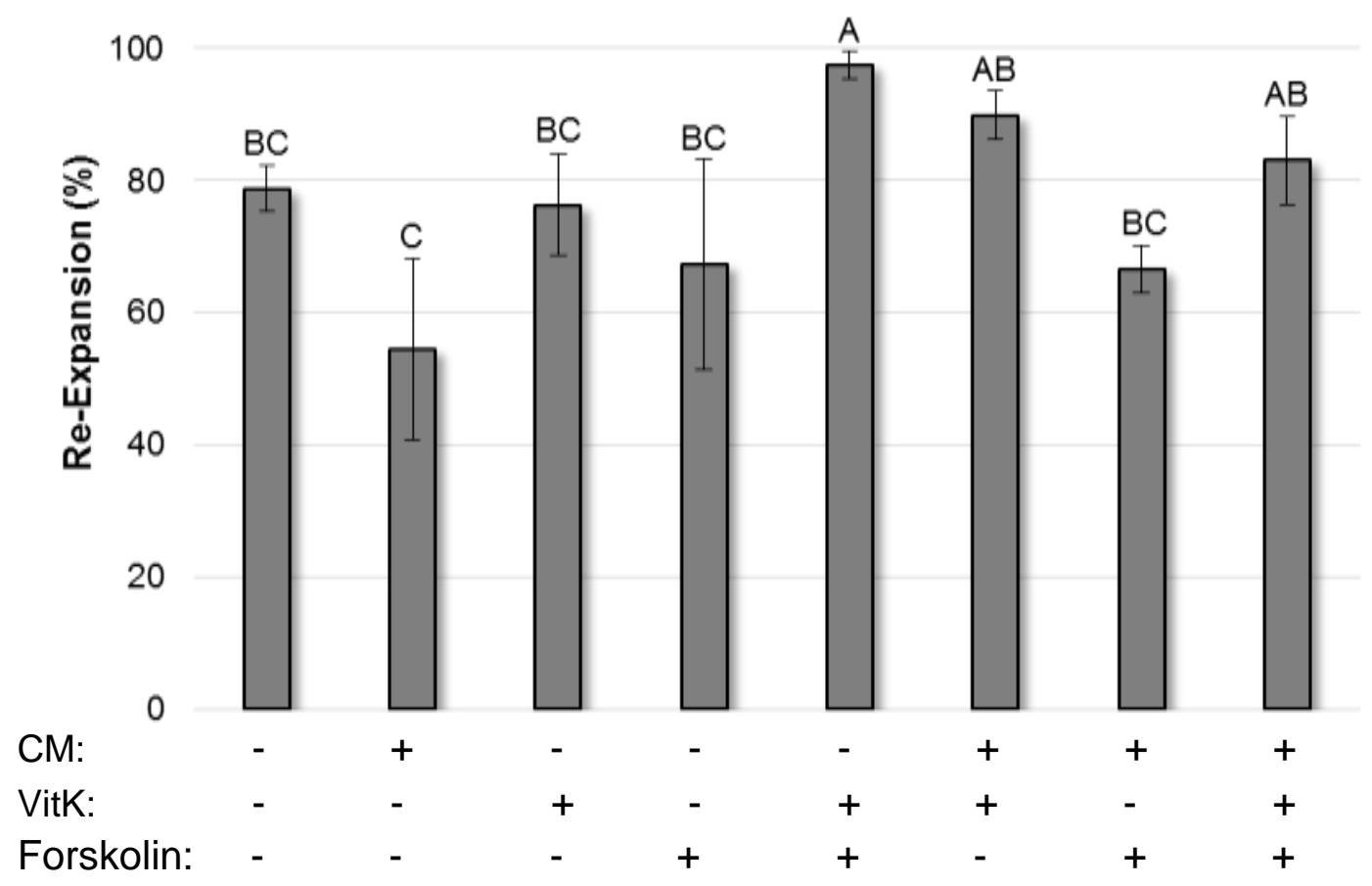

Figure 19. Re-expansion rate (Part 2). Post-thaw re-expansion rate was improved in embryos cultured in the presence of vitamin $\mathrm{K}_{2}$ and forskolin. $\mathrm{N}=4$ replicates. Error bars represent standard error of the mean, and values without common labels differ $(P<0.05)$. $\mathrm{CM}=$ conditioned media, $\mathrm{VitK}=$ vitamin $\mathrm{K}_{2}$. 


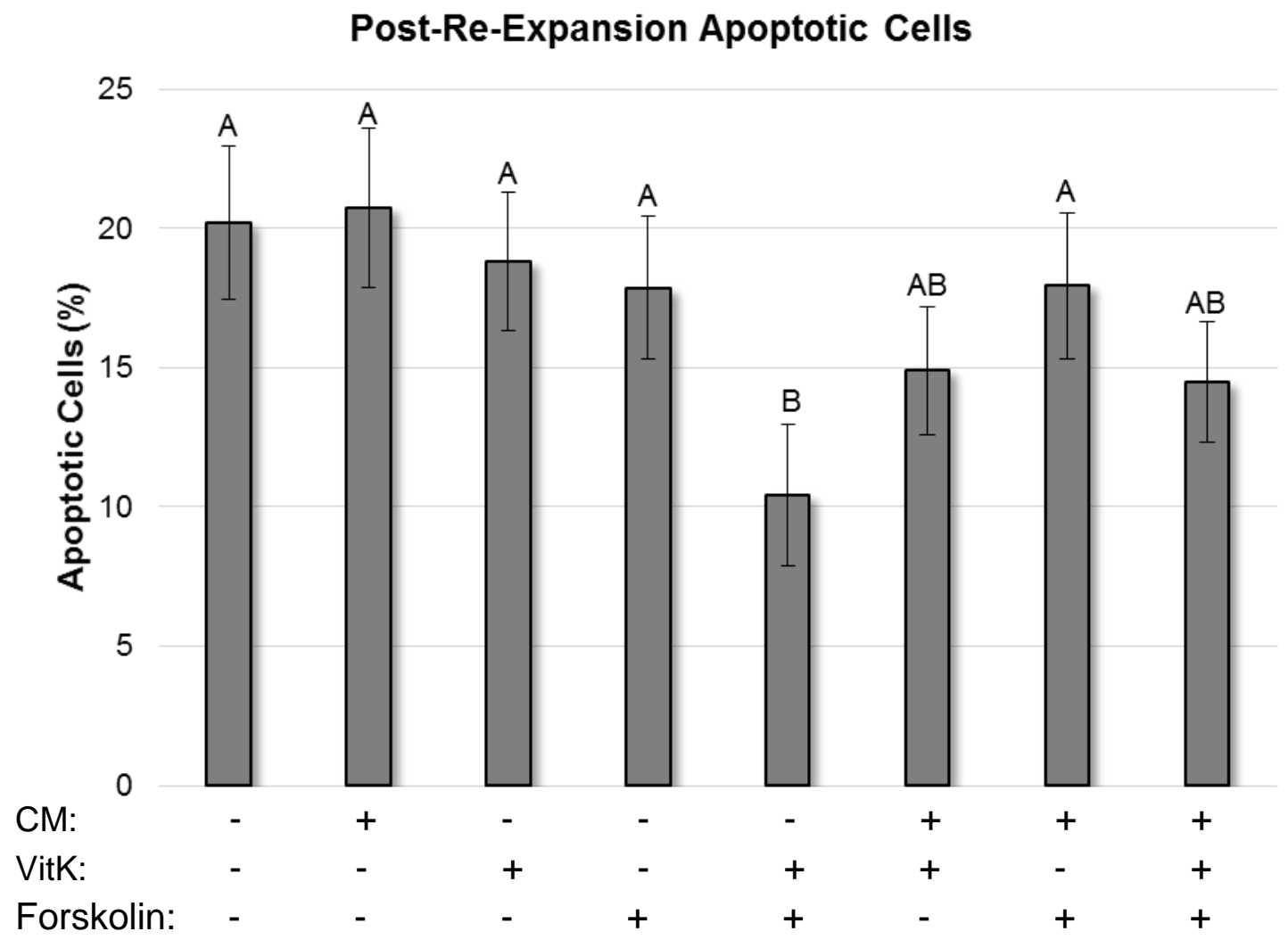

Figure 20. Percent apoptotic cells (Part 2). The percentage of apoptotic cells after reexpansion was decreased in embryos cultured with vitamin $K_{2}(V i t K)+$ forskolin $(n=38)$ compared to the control $(n=33)$. No difference was observed in the conditioned media (CM) $(n=30)$, VitK $(n=40)$, forskolin $(n=37), C M+$ VitK $(n=47), C M+$ forskolin $(n=36)$, or $C M+$ VitK + forskolin $(n=53)$ treatment groups. Error bars represent standard error of the mean, and values without common labels differ $(\mathrm{P}<0.05)$. 


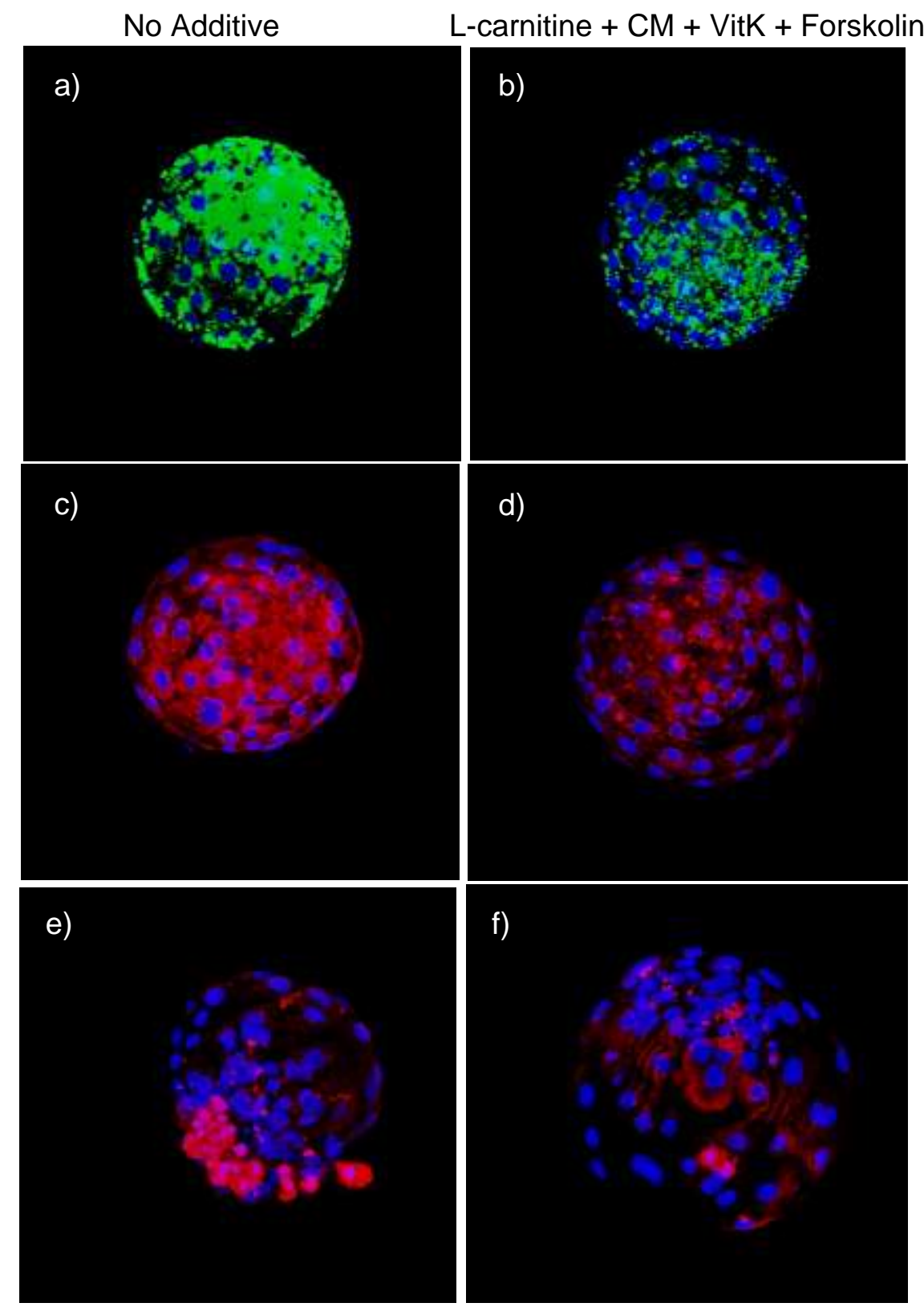

Figure 21. Representative images (Part 2). Images showing intracellular lipid (a-b) (Nile Red, green), mitochondrial polarity (c-d) (MitoTracker Red, red), and apoptotic cells (e-f) (TUNEL assay, red). In all images, nuclei are stained with DAPI (blue). Apoptotic cells are represented by an overlap of DAPI and TUNEL (e-f). Embryos in the left column of images were cultured with no additives (part 1), and embryos in the right column were cultured in the presence of all additives (L-carnitine + conditioned medium $(\mathrm{CM})+$ vitamkin $\mathrm{K}_{2}(\mathrm{VitK})$ + forskolin). 


\subsection{Discussion}

Mitochondrial dysfunction, increased cytoplasmic lipid accumulation, and elevated levels of reactive oxygen species have been described as the "fateful triad" of effects associated with bovine embryo in vitro culture (Prastowo et al., 2016). The cause, sequence, and interaction of these effects are poorly understood, however. As the embryo progresses through various stages of development, the functional role of mitochondria changes and these organelles mature (Houghton \& Leese, 2004; Tarazona et al., 2006; Jeremy G Thompson et al., 1996). Additionally, gene expression, enzyme activation, and energy demand are rapidly changing mechanisms within the preimplantation embryo (Gardner et al., 2000; Houghton \& Leese, 2004; H. J. Leese, 2012), lending complexity to the study of metabolic needs (Absalón-Medina et al., 2014). While it may be true that the embryo has a great deal of plasticity, the sweep of molecular events which follow a stressinduced reaction of the embryo to its environment can lead to abnormal fetal growth, modified placentation, and even abortion after transfer (H. J. Leese, 2012; J G Thompson, Kind, Roberts, Robertson, \& al, 2002). This highlights the significance and impact of generating a culture system which mimics the in vivo environment as closely as possible.

In part one of this experiment, L-carnitine reduced lipid content and improved postthaw re-expansion [Fig. 12-14]. The physiological role of this substance in transporting fatty acids across the inner mitochondrial membrane for $\beta$-oxidation and ATP production explains the reduction in lipid accumulation. Reduced intracellular lipid is associated with improved cryotolerance, which is consistent with the trend seen here. L-carnitine is also thought to act as an antioxidant by protecting the key enzymes involved in the cell's antioxidant defense system from peroxidative damage (Abdelrazik, Sharma, Mahfouz, \& Agarwa, 2009; Gülçin, 2006), which may add to the reason for improved cryotolerance observed. L-carnitine was kept as a constant factor for part two of this experiment because 
of the significant positive effect on cryopreservation seen and the well-explained mechanism of action to support the observations seen.

Complexity arises from the use of multiple metabolic regulators in parallel. While vitamin $\mathrm{K}_{2}$ and L-carnitine work through mechanisms focused within one part of the cell, the activation of adenylate cyclase by forskolin and the stimulation by growth factors from conditioned medium are non-specific mechanisms that can have an array of effects. Furthermore, when these media additives are combined, the interworking of their downstream pathways is unknown. For this reason, cryopreservation was chosen as the most relevant endpoint marker of embryo competence, supplemented by data regarding intracellular lipid content, mitochondrial polarity, blastocyst rates, and apoptotic cell percentage.

An interesting result was observed in the mitochondrial polarity of treatment groups in this experiment. After holding L-carnitine as a factor present in all treatment groups, there was no increase in mitochondrial polarity when vitamkin $K_{2}$, forskolin, or a combination of both was used. In fact, there was a decrease $(P<0.05)$ in polarity with all three (VitK + forskolin + L-carnitine) additives present [Fig. 18]. A similar phenomenon was observed in Pilot Study 2, wherein VitK and forskolin each increased mitochondrial polarity when used individually, but the effect was lost when the additives were used in combination with L-carnitine [Fig. 9].

A possible explanation for this is the presence of a feedback mechanism by which the mitochondrion can decrease activity in response to an excess of electron donors, or an accumulation of ATP (Prastowo et al., 2016). It is also possible that this feedback mechanism works through the activation of a transcription factor family known as hypoxiainducible factors, which are capable of dynamic responses to a variety of conditions and may be upregulated in response to the hypoxic environment in which embryos are cultured (Harvey, 2007). Alternatively, overwhelming the mitochondria by providing an excess of 
fatty acids can result in an increase in ROS, which disturbs $\beta$-oxidation and reduces mitochondrial activity (Prastowo et al., 2016). The exact mechanism by which disturbed $\beta$-oxidation decreases mitochondrial activity is unknown, however (Prastowo et al., 2016).

This effect was seen in treatment groups containing L-carnitine because this molecule is required for function of the rate-limiting enzyme in fatty acid metabolism: CPTIB (Sutton-McDowall et al., 2012). Increasing the presence of free fatty acids through forskolin supplementation, or mitochondrial efficiency through CoQ or VitK supplementation can only increase the function of the electron transport chain to a certain extent. Increasing the availability of a rate-limiting molecule may provide the mitochondrion with such an abundance of electron donating molecules that this feedback mechanism is activated and mitochondrial activity decreases.

Defining markers of superior embryo quality and developmental competence is a topic that is not widely agreed upon. When evaluating mitochondrial dysfunction, mitochondrial polarity has been used as a marker of electron transport chain function. Studies have interpreted a greater inner mitochondrial membrane potential as an indication of increased mitochondrial function and improved embryo viability (BaldocedaBaldeon et al., 2014). The validity of this marker has been questioned, however, as it has been suggested that mitochondria which are actively undergoing ATP synthesis would be experiencing a decrease in mitochondrial polarity (Diaz et al., 1999; Van Blerkom, 2004, 2009). Additionally, mitochondrial polarity changes depending on the type of cell to cell interactions present (Van Blerkom, 2009). This trend can be seen by the formation of gap junctions during the blastocyst stage coinciding with a decrease in mitochondrial polarity (Van Blerkom, 2009).

As an alternate approach to this marker, perhaps it is not the activity of mitochondria that should be evaluated, but rather the efficiency. In this context, a more relevant mark of mitochondrial function may be ROS generation. High levels of ROS are 
associated with improper oxidative phosphorylation, lipid accumulation, and decreased embryo viability (Dumollard et al., 2009; Houghton \& Leese, 2004; Prastowo et al., 2016; M. Takahashi et al., 2000). Because ROS are a downstream effect of mitochondrial dysfunction and have a direct link to perturbations in cellular function (Prastowo et al., 2016; Van Blerkom, 2009), measurement of ROS may be a better marker of mitochondrial function. The trend seen from this experiment supports this notion, as an increase in mitochondrial polarity did not correlate with an increase in cryotolerance [Fig. 13, 14, 17, \&20].

Adding to the doubt of using increased mitochondrial polarity as a marker of competence is the quiet embryo hypothesis. In this theory, embryos with a quieter metabolism are subject to fewer damages to the genome, transcriptome, and proteome of cells as a result of lower ROS generation (Henry J. Leese, 2002). It is believed that embryos under cellular stress require more ATP for cellular repair mechanisms than more competent embryos (H. J. Leese, 2012; Henry J. Leese, 2002).

It was found that a combination of L-carnitine, vitamin $\mathrm{K}_{2}$, and forskolin had the most positive effect on cryopreservation survival, however, conditioned medium provided an increase in the percentage of embryos reaching the blastocyst stage. Because cryotolerance was chosen as the ultimate marker for embryo quality, L-carnitine in combination with only vitamin $\mathrm{K}_{2}$ and forskolin was concluded to generate the most competent embryo. As testament to the quality of embryos in this treatment group, the percentage of apoptotic cells present in the re-expanded frozen embryos was decreased, indicating a superior ability of the cells within these embryos to withstand freezing. The rescue of cells from mitochondrial dysfunction can prevent apoptosis, which is the objective of using VitK in embryo culture (Baldoceda-Baldeon et al., 2014; Vos et al., 2012). A reduced percentage of apoptotic cells as well as increased cryotolerance indicates a rescue of these embryos from mitochondrial dysfunction, suggesting a re- 
evaluation of the use of mitochondrial polarity on stage 7 blastocysts as a marker for embryo quality. L-carnitine, VitK, and forskolin are believed to work through various pathways which together help to mitigate the negative artifacts of in vitro culture. Future studies should more precisely evaluate the pathways of action of these metabolic regulators and investigate biologically relevant substitutes. 


\section{Chapter 6. Concluding Remarks}

This research contributes to the vast effort of understanding preimplantation embryo energy metabolism and developmental viability. While these studies lend insight into the effects of metabolic regulators during culture from the 1-cell to blastocyst stage, a major limitation of this research is that data collection stops in vitro. A more encompassing portrayal of the developmental competence of these embryos would involve trials followed through to the pregnancy and post-partum stages so that pregnancy rate and normalcy of offspring can be assessed. In fact, this seems to be a pitfall of most current data regarding embryo metabolism (H. J. Leese, 2012). For evaluating development, emphasis is typically placed on the percentage of embryos that reach the blastocyst stage rather than the normality of these cultured embryos (Barnett \& Bavister, 1996).

In the more immediate future, this research can be applied to the combination of an optimized culture system with an optimized cryopreservation method. Efforts have been focused on the improvement of in vitro culture conditions because the negative outcome of cryopreservation of IVP embryos results from the deviant qualities of these embryos in relation to in vivo derived embryos (Seidel, 2006). Modifying cryopreservation methods to fit the cells being cryopreserved will also improve cryopreservation outcome (Seidel, 2006). Additionally, the intrinsic quality of the oocyte is the main factor affecting blastocyst yield (Rizos, 2002). As such, studies combining improvement in oocyte maturation in vitro with embryo culture in vitro will advance the quality and cryotolerance of IVP embryos.

Evaluating the normalcy of IVP embryos requires a thorough understanding of in vivo embryo metabolism as well as the molecular dialog between the embryo and the female reproductive tract, both of which are still lacking $(H$. J. Leese, 2012). Knowledge of embryo metabolism within the reproductive tract will allow for the utilization of more relevant and effective biomarkers of embryo health. An understanding of the molecular 
dialog between the embryo and its niche environment will aid in the tracking and prevention of epigenetic changes that have long term developmental consequences. Ultimately, research such as this helps to improve the success of assisted reproductive technologies and advance breeding efficiency within the dairy industry. 


\section{REFERENCES}

Abdelrazik, H., Sharma, R., Mahfouz, R., \& Agarwa, A. (2009). L-carnitine decreases DNA damage and improves the in vitro blastocyst development rate in mouse embryos. Reproductive Biology, 91(2), 589-596.

Abe, H., Yamashita, S., Satoh, T., \& Hoshi, H. (2002). Accumulation of Cytoplasmic Lipid Droplets in Bovine Embryos and Cryotolerance of Embryos Developed in Different Culture Systems Using Serum-Free or Serum-Containing Media. Molecular Reproduction and Development, 61(1), 57-66.

Absalón-Medina, V. a., Butler, W. R., \& Gilbert, R. O. (2014). Preimplantation embryo metabolism and culture systems: Experience from domestic animals and clinical implications. Journal of Assisted Reproduction and Genetics, 31(4), 393-409.

Baldoceda, L., Gagne, D., Ferreira, C. R., \& Robert, C. (2015). Genetic influence on the reduction in bovine embyo lipid content by L-carnitine. Reproduction, Fertility, and Development.

Baldoceda, L., Gilbert, I., Gagne, D., Vigneault, C., Blondin, P., Ferreira, C. R., \& Robert, C. (2016). Breed-specific factors influence embryonic lipid composition: comparison between Jersey and Holstein. Reproduction, Fertility and Development, 28(8), 1185.

Baldoceda-Baldeon, L. M., Gagne, D., Vigneault, C., Blondin, P., \& Robert, C. (2014). Improvement of bovine in vitro embryo production by vitamin K2 supplementation. Reproduction, 148(5), 489-497.

Barceló-Fimbres, M., \& Seidel, G. E. (2007a). Effects of Either Glucose or Fructose and Metabolic Regulators on Bovine Embryo Development and Lipid Accumulation In Vitro. Molecular Reproduction and Development, 74, 1406-1418. 
Barceló-Fimbres, M., \& Seidel, G. E. (2007b). Effects of Fetal Calf Serum , Phenazine Ethosulfate and Either Glucose or Fructose During In Vitro Culture of Bovine Embryos on Embryonic Development After Cryopreservation. Molecular Reproduction and Development, 74, 1395-1405.

Barnett, D. K., \& Bavister, B. D. (1996). What is the relationship between the metabolism of preimplantation embryos and their developmental competence? Molecular Reproduction and Development, 43(1), 105-133.

Baumann, C. G., Morris, D. G., Sreenan, J. M., \& Leese, H. J. (2007). The quiet embryo hypothesis: molecular characteristics favoring viability. Molecular Reproduction and Development, 74(10), 1345-1353.

Bavister, B. (1999). Glucose and the culture of human embryos. Fertility and Sterility, 72(2), 233-234.

Bavister, B. D., \& Squirrell, J. M. (2000). Mitochondrial distribution and function in oocytes and early embryos. Human Reproduction, 15, 189-198.

Bentinger, M., Tekle, M., \& Dallner, G. (2010). Coenzyme Q - Biosynthesis and functions. Biochemical and Biophysical Research Communications, 396(1), 74-79.

Blondin, P. (2015). Status of embryo production in the world. Animal Reproduction, 12(3), 356-358.

Boveris, A., Cadenas, E., \& Stoppani, A. O. (1976). Role of ubiquinone in the mitochondrial generation of hydrogen peroxide. The Biochemical Journal, 156(2), 435-44.

Chen, H., Ho, H., Chen, S., Chao, K., Lin, H., Huang, S., ... Yang, Y. (1994). Peptides Extracted from Vero Cell Cultures Overcome the Blastocyst Block of Mouse Embryos in a Serum-Free Medium. Journal of Assisted Reproduction and Genetics, 11(3), 
165-171.

Cummins, J. M. (2004). The role of mitochondria in the establishment of oocyte functional competence. European Journal of Obstetrics and Gynecology, 115, 23-29.

de Souza, D. K., Salles, L. P., \& Rosa e Silva, A. A. M. (2015). Aspects of energetic substrate metabolism of in vitro and in vivo bovine embryos. Brazilian Journal of Medical and Biological Research , 48(3), 191-7.

Diaz, G., Setzu, M. D., Zucca, a, Isola, R., Diana, a, Murru, R., ... Gremo, F. (1999). Subcellular heterogeneity of mitochondrial membrane potential: relationship with organelle distribution and intercellular contacts in normal, hypoxic and apoptotic cells. Journal of Cell Science, 112, 1077-84.

Dobrinsky, J. (2002). Advancements in cryopreservation of domestic animal embryos. Theriogenology, 57(1), 285-302.

Dumollard, R., Carroll, J., Duchen, M. R., Campbell, K., \& Swann, K. (2009). Mitochondrial function and redox state in mammalian embryos. Semin Cell Dev Biol, 20(3), 346353.

Dumollard, R., Duchen, M., \& Carroll, J. (2007). The Role of Mitochondrial Function in the Oocyte and Embryo. Current Topics in Developmental Biology, 77, 21-49.

Dunning, K. R., Russell, D. L., \& Robker, R. L. (2014). Lipids and oocyte developmental competence: the role of fatty acids and $\beta$-oxidation. Reproduction, 148(1), R15-R27.

Enright, B. P., Lonergan, P., Dinnyes, A., Fair, T., Ward, F. A., Yang, X., \& Boland, M. P. (2000). Culture of in vitro produced bovine zygotes in vitro vs in vivo: Implications for early embryo development and quality. Theriogenology, 54(5), 659-673.

Fahy, G. M., MacFarlane, D. R., Angell, C. A., \& Meryman, H. T. (1984). Vitrification as an 
approach to cryopreservation. Cryobiology, 21(4), 407-426.

Fair, T., Lonergan, P., Dinnyes, a., Cottell, D. C., Hyttel, P., Ward, F. a., \& Boland, M. P. (2001). Ultrastructure of bovine blastocysts following cryopreservation: Effect of method of blastocyst production. Molecular Reproduction and Development, 58(2), $186-195$.

Fakruzzaman, M., Ghanem, N., Bang, J.-I., Ha, A.-N., Lee, K.-L., Sohn, S.-H., ... Kong, I.K. (2015). Effect of peroxiredoxin II on the quality and mitochondrial activity of preimplantation bovine embryos. Animal Reproduction Science, 159, 172-183.

Ferguson, E. M., \& Leese, H. J. (1999). Triglyceride content of bovine oocytes and early embryos. Journal of Reproduction and Fertility, 116(2), 373-378.

Ferguson, E. M., \& Leese, H. J. (2006). A Potential Role for Triglyceride as an Energy Source During Bovine Oocyte Maturation and Early Embryo Development. Molecular Reproduction and Development, 73, 1195-1201.

Fischer, B., \& Bavister, B. D. (1993). Oxygen tension in the oviduct and uterus of rhesus monkeys, hamsters and rabbits. Journal of Reproduction and Fertility, 99(2), 673679.

Gad, a., Hoelker, M., Besenfelder, U., Havlicek, V., Cinar, U., Rings, F., ... Tesfaye, D. (2012). Molecular Mechanisms and Pathways Involved in Bovine Embryonic Genome Activation and Their Regulation by Alternative In Vivo and In Vitro Culture Conditions. Biology of Reproduction, 87(4), 1-13.

Gardner, D. K., Pool, T. B., \& Lane, M. (2000). Embryo nutrition and energy metabolism and its relationship to embryo growth, differentiation, and viability. Seminars in Reproductive Medicine, 18(2), 205-18. 
Gülçin, I. (2006). Antioxidant and antiradical activities of L-carnitine. Life Sciences, 78(8), 803-811.

Gupta, A., Singh, J., \& Anzar, M. (2016). Effect of cryopreservation technique and season on the survival of in vitro produced cattle embryos. Animal Reproduction Science, 164, 162-168.

Harvey, A. J. (2007). The role of oxygen in ruminant preimplantation embryo development and metabolism. Animal Reproduction Science, 98(1-2), 113-128.

Hasler, J. F. (2001). Factors affecting frozen and fresh embryo transfer pregnancy rates in cattle. Theriogenology, 56(1), 1401-1415.

Hasler, J. F. (2014). Forty years of embryo transfer in cattle: A review focusing on the journal Theriogenology, the growth of the industry in North America, and personal reminisces. Theriogenology, 81(1), 152-169.

Houghton, F. D., \& Leese, H. J. (2004). Metabolism and developmental competence of the preimplantation embryo. European Journal of Obstetrics and Gynecology, 115, 92-96.

Hyttel, P., Fair, T., Callesen, H., \& Greve, T. (1997). Oocyte growth, capacitation and final maturation in cattle. Theriogenology, 47, 23-32.

Jeong, W. J., Cho, S. J., Lee, H. S., Deb, G. K., Lee, Y. S., Kwon, T. H., \& Kong, I. K. (2009). Effect of cytoplasmic lipid content on in vitro developmental efficiency of bovine IVP embryos. Theriogenology, 72(4), 584-589.

Kwong, L. K., Kamzalov, S., Rebrin, I., Bayne, A. C. V, Jana, C. K., Morris, P., ... Sohal, R. S. (2002). Effects of coenzyme Q10 administration on its tissue concentrations, mitochondrial oxidant generation, and oxidative stress in the rat. Free Radical Biology 
and Medicine, 33(5), 627-638.

Lazzari, G. (2002). Cellular and Molecular Deviations in Bovine In Vitro-Produced Embryos Are Related to the Large Offspring Syndrome. Biology of Reproduction, 67(3), 767-775.

Leese, H. J. (2002). Quiet please, do not disturb: A hypothesis of embryo metabolism and viability. BioEssays, 24(9), 845-849.

Leese, H. J. (2012). Metabolism of the preimplantation embryo: 40 years on. Reproduction, 143(4), 417-427.

Lim, K. T., Jang, G., Ko, K. H., Lee, W. W., Park, H. J., Kim, J. J., ... Kang, S. K. (2007). Improved in vitro embryo development and increased efficiency in producing viable calves using defined media. Theriogenology, 67(2), 293-302.

Londos, C., Brasaemle, D. L., Schultz, C. J., Adler-wailes, D. C., Levin, D. M., Kimmel, A. R., \& Rondinone, C. M. (1999). On the Control of Lipolysis in Adipocytes. Annals New York Academy of Sciences, 892, 155-168.

Maeda, J., Kotsuji, F., Negami, A., Kamitani, N., \& Tominaga, T. (1996). In Vitro Development of Bovine Embryos in Conditioned Media from Bovine Granulosa Cells and Vero Cells Cultured in Exogenous Protein- and Amino Acid-Free Chemically Defined Human Tubal Fluid Medium. Biology of Reproduction, 93654, 930-936.

Mapletoft, R. J. (2013). History and perspectives on bovine embryo transfer. Animal Reproduction, 10(3), 168-173.

Massip, A. (2001). Cryopreservation of embryos of farm animals. Reproduction in Domestic Animals, 36(2), 49-55.

Mazur, P. (1963). Kinetics of Water Loss From Cells At Subzero Temperatures and the 
Likelihood of Intracellular Freezing. The Journal of General Physiology, 47, 347-369.

Mcevoy, T. G., Coull, G. D., Broadbent, P. J., Hutchinson, J. S. M., \& Speake, B. K. (2000). Fatty acid composition of lipids in immature cattle, pig and sheep oocytes with intact zona pellucida. Journal of Reproduction and Fertility, 118, 163-170.

McEvoy, T. G., Robinson, J. J., \& Sinclair, K. D. (2001). Developmental consequences of embryo and cell manipulation in mice and farm animals. Reproduction, 122(4), 507518.

Men, H., Agca, Y., Riley, L. K., \& Critser, J. K. (2006). Improved survival of vitrified porcine embryos after partial delipation through chemically stimulated lipolysis and inhibition of apoptosis. Theriogenology, 66, 2008-2016.

Moore, K., \& Thatcher, W. W. (2006). Major Advances Associated with Reproduction in Dairy Cattle. Journal of Dairy Science, 89(4), 1254-1266.

Paschoal, D. M., Sudano, M. J., Guastali, M. D., Dias Maziero, R. R., Crocomo, L. F., Oña Magalhães, L. C., ... da Cruz Landim-Alvarenga, F. (2012). Forskolin effect on the cryosurvival of in vitro-produced bovine embryos in the presence or absence of fetal calf serum. Zygote, 22(2), 146-57.

Perry, G. (2014). IETS 2013 Statistics of embryo collection and transfer in domestic farm animals. International Embryo Transfer Society (IETS) (Vol. 2).

Plante, L., \& King, W. A. (1994). Light and Electron Microscopic Analysis of Bovine Embryos Derived by in Vitro and in Vivo Fertilization. Journal of Assisted Reproduction and Genetics, 11(10), 515-529.

Prastowo, S., Amin, A., Rings, F., Held, E., Wondim, D. S., Gad, A., ... Hoelker, M. (2016). Fateful triad of reactive oxygen species, mitochondrial dysfunction and lipid 
accumulation is associated with expression outline of the AMP-activated protein kinase pathway in bovine blastocysts. Reproduction, Fertility, and Development.

Rieger, D. (1992). Relationships between energy metabolism and development of early mammalian embryos. Theriogenology, 37(1), 75-93.

Rizos, D. (2002). Bovine Embryo Culture in the Presence or Absence of Serum: Implications for Blastocyst Development, Cryotolerance, and Messenger RNA Expression. Biology of Reproduction, 68(1), 236-243.

Robertson, I., \& Nelson, R. (1998). Certification and identification of the embryo. In D. A. Stringfellow \& S. M. Seidel (Eds.), Manual of the International Embryo Transfer Society (IETS) (3rd ed., pp. 103-116). Savory, IL, USA: IETS.

Sanches, B. V., Marinho, L. S. R., Filho, B. D. O., Pontes, J. H. F., Basso, A. C., Meirinhos, M. L. G., ... Seneda, M. M. (2013). Cryosurvival and pregnancy rates after exposure of IVF-derived Bos indicus embryos to forskolin before vitrification. Theriogenology, 80(4), 372-377.

Saragusty, J., \& Arav, A. (2011). Current progress in oocyte and embryo cryopreservation by slow freezing and vitrification. Reproduction, 141(1), 1-19.

Schneider, H., Lemasters, J. J., \& Hackenbrock, C. R. (1982). Lateral Diffusion of Ubiquinone During Electron-Transfer in Phospholipid-Enriched and UbiquinoneEnriched Mitochondrial-Membranes. Journal of Biological Chemistry, 257(18), 789793.

Seamon, K. B., Padgett, W., \& Daly, J. W. (1981). Forskolin: unique diterpene activator of adenylate cyclase in membranes and in intact cells. Proceedings of the National Academy of Sciences of the United States of America, 78(6), 3363-3367. 
Seidel, G. E. (2006). Modifying oocytes and embryos to improve their cryopreservation. Theriogenology, 65(1), 228-235.

Seshagiri, P. B., \& Bavister, B. D. (1991). Glucose and Phosphate Inhibit Respiration and Oxidative Metabolism in Cultured Hamster Eight-Cell Embryos : Evidence for the " Crabtree Effect ." Molecular Reproduction and Development, 30, 105-111.

Shehab-El-Deen, M. A., Leroy, J. L. M. R., Maes, D., \& Van Soom, A. (2009). Cryotolerance of bovine blastocysts is affected by oocyte maturation in media containing palmitic or stearic acid. Reproduction in Domestic Animals, 44(1), 140142.

Stojkovic, M., Westesen, K., Zakhartchenko, V., Stojkovic, P., Boxhammer, K., \& Wolf, E. (1999). Coenzyme $Q(10)$ in submicron-sized dispersion improves development, hatching, cell proliferation, and adenosine triphosphate content of in vitro-produced bovine embryos. Biology of Reproduction, 61, 541-547.

Sturmey, R. G., Brison, D. R., \& Leese, H. J. (2008). Symposium : Innovative techniques in human embryo viability assessment Assessing embryo viability by measurement of amino acid turnover. Reproductive BioMedicine 17(4), 486-496.

Sturmey, R., Reis, A., Leese, H., \& McEvoy, T. (2009). Role of Fatty Acids in Energy Provision During Oocyte Maturation and Early Embryo Development. Reproduction in Domestic Animals, 44, 50-58.

Sudano, M. J., Paschoal, D. M., da Silva Rascado, T., Magalhães, L. C. O., Crocomo, L. F., de Lima-Neto, J. F., \& da Cruz Landim-Alvarenga, F. (2011). Lipid content and apoptosis of in vitro-produced bovine embryos as determinants of susceptibility to vitrification. Theriogenology, 75(7), 1211-1220. 
Sudano, M., Paschoal, D., \& Maziero, R. (2013). Improving postcryopreservation survival capacity: an embryo-focused approach. Animal Reproduction Science, 10(3), 160167.

Sun, I. L., Sun, E. E., \& Crane, F. L. (1992). Stimulation of serum-free cell proliferation by coenzyme Q. Biochemical and Biophysical Research Communications, 189(1), 813.

Sutton-McDowall, M. L., Feil, D., Robker, R. L., Thompson, J. G., \& Dunning, K. R. (2012). Utilization of endogenous fatty acid stores for energy production in bovine preimplantation embryos. Theriogenology, 77(8), 1632-41.

Takahashi, M., Keicho, K., Takahashi, H., Ogawa, H., Schultz, R., \& Okano, A. (2000). Effect of oxidative stress on development and DNA damage in in-vitro cultured bovine embryos by comet assay. Theriogenology, 54, 137-145.

Takahashi, T., Inaba, Y., Somfai, T., Kaneda, M., Geshi, M., Nagai, T., \& Manabe, N. (2013). Supplementation of culture medium with L-carnitine improves development and cryotolerance of bovine embryos produced in vitro. Reproduction, Fertility and Development, 25(4), 589-599.

Tarazona, a M., Rodríguez, J. I., Restrepo, L. F., \& Olivera-Angel, M. (2006). Mitochondrial activity, distribution and segregation in bovine oocytes and in embryos produced in vitro. Reproduction in Domestic Animals, 41(1), 5-11.

Tavares, L. M. T., Feitosa, W. B., de Oliveira, V. P., Nichi, M., Assumpcao, M. E. O. D., \& Visintin, J. A. (2011). In vitro development of bovine embryos cultured under different fetal calf serum concentrations and cell types. Brazilian Journal of Veterinary Research and Animal Science, 48(1), 38-45. 
Telford, N. A., Watson, A. J., \& Schultz, G. A. (1990). Review Article: Transition From Maternal to Embryonic Control in Early Mammalian Development: A Comparison of Several Species. Molecular Reproduction and Development, 26, 90-100.

Thompson, J. G. (2000). In vitro culture and embryo metabolism of cattle and sheep embryos - A decade of achievement. Animal Reproduction Science, 60-61, 263275.

Thompson, J. G., Kind, K. L., Roberts, C. T., Robertson, S. A., \& al, et. (2002). Epigenetic risks related to assisted reproductive technologies: Short-and long-term consequences for the health of children conceived through assisted reproduction Human Reproduction, 17(11), 2783-2786.

Thompson, J. G., Partridge, R. J., Houghton, F. D., Cox, C. I., \& Leese, H. J. (1996). Oxygen uptake and carbohydrate metabolism by in vitro derived bovine embryos. Reproduction, 106(1993), 299-306.

Turrens, J. F. (1997). Superoxide production by the mitochondrial respiratory chain. Bioscience Reports, 17(1), 3-8.

Ushijima, H., Yamakawa, H., \& Nagashima, H. (1999). Cryopreservation of Bovine PreMorula-Stage In Vitro Matured / In Vitro Fertilized Embryos after Delipidation and before Use in Nucleus Transfer. Biology of Reproduction, 60, 534-539.

Vajta, G., \& Kuwayama, M. (2006). Improving cryopreservation systems. Theriogenology, 65(1), 236-244.

Van Blerkom, J. (2004). Mitochondria in human oogenesis and preimplantation embryogenesis: Engines of metabolism, ionic regulation and developmental competence. Reproduction, 128, 269-280. 
Van Blerkom, J. (2009). Mitochondria in early mammalian development. Seminars in Cell and Developmental Biology, 20(3), 354-364.

Vos, M., Esposito, G., Edirisinghe, J. N., Vilain, S., Haddad, D. M., Slabbaert, J. R., ... Verstreken, P. (2012). Vitamin K2 is a mitochondrial electron carrier that rescues Pink1 deficiency. Science, 336(June), 1306-1311.

Whittingham, D. G., Leibo, S. P., \& Mazur, P. (1972). Survival of Mouse Embryos Frozen to $-196^{\circ}$ and $-269^{\circ} \mathrm{C}$. Science, $178(4059), 411-414$.

Wilmut, I., \& Rowson, L. (1973). Experiments on the low-temperature preservation of cow embryos. Veterinary Record, 92(26), 686-690.

Young, L. E., Sinclair, K. D., \& Wilmut, I. (1998). Large offspring syndrome in cattle and sheep. Reviews of Reproduction, 3, 155-163. 